\title{
Investigating the Kinetics of Blood Coagulation Using High-Frequency Ultrasound
}

\author{
by \\ Morgan Maher \\ B.Sc., University of British Columbia, 2017
}

\begin{abstract}
A thesis presented to Ryerson University
in partial fulfillment of the requirements for the degree of

MASTER OF SCIENCE

in the program of

BiomedicAl Physics
\end{abstract}

Toronto, Ontario, Canada, 2019

(c) Morgan Maher, 2019 


\section{Author's Declaration}

I hereby declare that I am the sole author of this thesis. This is a true copy of the thesis, including any required final revisions, as accepted by my examiners.

I authorize Ryerson University to lend this thesis to other institutions or individuals for the purpose of scholarly research.

I further authorize Ryerson University to reproduce this thesis by photocopying or by other means, in total or in part, at the request of other institutions or individuals for the purpose of scholarly research.

I understand that my thesis may be made electronically available to the public.

Morgan Maher 


\title{
Abstract
}

\section{Investigating the Kinetics of Blood Coagulation Using High-Frequency Ultrasound}

\author{
Morgan Maher \\ Master of Science, Biomedical Physics \\ Department of Physics, Ryerson University, 2019
}

Blood circulation requires regulated clot formation and breakdown to prevent blood loss following an injury and to ensure that clots do not form and circulate within the vasculature. Known as hemostasis, this delicate balance between coagulant and anti-coagulant pathways can be disrupted by disease, medication, or trauma, and may lead to morbidity or mortality. Current in vitro hemostatic tests have shown promise as tools for diagnosis and risk assessment in certain disorders. However, these tests are limited in their ability to assess the complete hemostatic process or are restricted to studies of blood plasma. In this work, high frequency ultrasound is proposed as a method of assessing hemostasis in whole blood samples. A system was developed and experiments were performed by monitoring acoustic changes in mouse blood during coagulation. Blood cell motion and frequency dependant changes in ultrasound intensity were found to be sensitive to the kinetics of clot formation. 


\section{Acknowledgements}

I would like to start off by thanking my supervisor Dr. Michael Kolios. Your guidance and encouragement over the last two years have been invaluable, and seeing your sense of curiosity and appreciation for science has been truly inspiring. Additionally, I cannot thank you enough for the work that you put in over the last several months to help me complete this project. To Dr. Mark McVey, thank you for always pushing me to do more, and for all of the ideas that came up in our many discussions. It has been a pleasure to work with you and to have you as a member on my committee. To the final member of my committee, Dr. Carl Kumaradas, thank you for challenging me to think critically and pointedly about the direction of the project, and for providing helpful advice along the way (in both medical physics and volleyball).

From within the Kolios group, Mike Moore, thank you for teaching me to use the SASAM and for being a wonderful scientific role model in the early stages of this project. To Elizabeth, thank you for all of the work that you put in to keep the lab running smoothly, and for your willingness to help me and to answer my questions whenever they came up. I would also like to extend my sincere thanks to some of the friends I have made within the group: Charlotte for your encouragement, kind words, and for getting me involved with the BPGSU, Dana for your inspiring love of science and science outreach, Grace for your continued support, understanding and ice cream breaks, and Hossein for our many discussions on physics, politics, and photography. To all the other members of the Kolios group, thank you all for your input, comments and discussions over the last two years.

To my family, I truly can't express enough gratitude. Mom and dad I wouldn't be anywhere near where I am today without your continued support, encouragement and love. To Erin and Gaëlle, you make me strive to be the best version of myself and remind me that there are so many important things in life outside of the world of academia that I sometimes get caught up in. And to the rest of my family, knowing that you are there cheering me along from the sidelines has made all the difference. Thank you all.

Finally, to Chris I can’t thank you enough. Your love, support and help along the way made this work possible. 


\section{Table of Contents}

Author's Declaration . . . . . . . . . . . . . . . . . ii

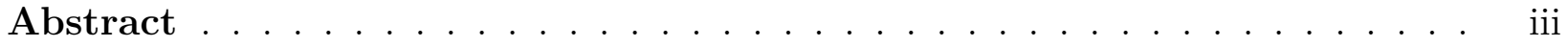

Acknowledgements ...................... iv

List of Tables . . . . . . . . . . . . . . . . . . . . . . viii

List of Figures . . . . . . . . . . . . . . . . . . . . ix

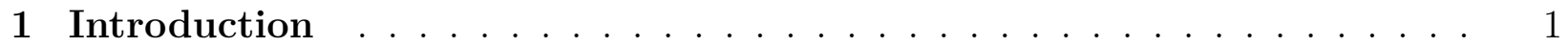

1.1 Hemostasis and Disease . . . . . . . . . . . . . . . . . 1

1.2 Coagulation Tests . . . . . . . . . . . . . . . . 2

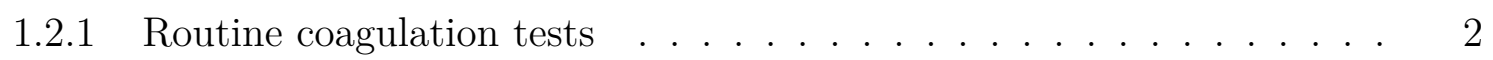

1.2.2 Global coagulation tests ...................... 3

1.3 Hemostasis and ultrasound . . . . . . . . . . . . . . . . . 5

1.4 Hypothesis and outline of the thesis . . . . . . . . . . . . . . 5

2 Background and Theory ......................... 6

2.1 Fundamentals of Ultrasound . . . . . . . . . . . . . . . 6

2.1 .1 Acoustic waves ....................... 6

2.1.2 Properties of acoustic media . . . . . . . . . . . . . 7

2.1.3 Transducers . . . . . . . . . . . . . . . . . . . . . 10

2.1.4 Imaging modalities . . . . . . . . . . . . . . . . . . . . . . 11

2.2 Hemostasis . . . . . . . . . . . . . . . . . . . . . 13

2.2.1 The hemostatic system _. . . . . . . . . . . . . . 13

2.2.2 Hemostasis in Mice . . . . . . . . . . . . . . . . . . . . . 18

2.3 Ultrasound and blood coagulation . . . . . . . . . . . . . . . 19

2.3.1 Backscatter measurements . . . . . . . . . . . . . 20

2.3.2 Speed of sound . . . . . . . . . . . . . . . 20

2.3.3 Attenuation and spectral intensity . . . . . . . . . . . . . 23 
$2.3 .4 \quad$ M-mode imaging . . . . . . . . . . . . . . . . . . . . . . . . . 24

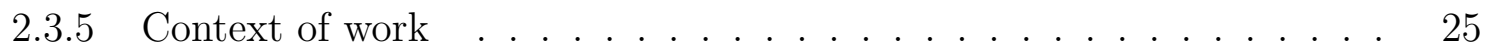

3 Materials and Methods _. . . . . . . . . . . . . . . . . . 26

3.1 Acoustic Acquisition System _. . . . . . . . . . . . . 26

3.1 .1 Conventional use . . . . . . . . . . . . . . . . . . . . 26

3.1 .2 System modifications . . . . . . . . . . . . . . . 26

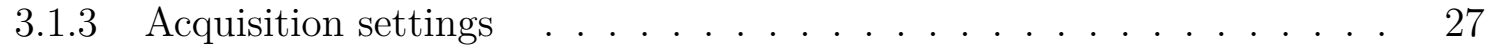

3.1 .4 Transducer specifications $\ldots \ldots \ldots \ldots$

3.1 .5 Typical acquisitions . . . . . . . . . . . . . . . . . . . 29

3.2 Experimental refinement . . . . . . . . . . . . . . . . . . . . . 33

3.2 .1 Meniscus . . . . . . . . . . . . . . . . . . . . 33

3.2 .2 Evaporation . . . . . . . . . . . . . . . . . . . . 34

3.2 .3 Temperature . . . . . . . . . . . . . . . . . . . . . 34

3.2 .4 Acquisition automation . . . . . . . . . . . . . . . 34

3.2 .5 Well cap . . . . . . . . . . . . . . . . . 35

3.3 Coagulation and Fybrinolytic Assay _ . . . . . . . . . . . 36

3.3 .1 Buffer preparations . . . . . . . . . . . . . . 36

3.3 .2 Stock enzyme dilutions . . . . . . . . . . . . . . . . . 37

3.3 .3 Acellular clot composition . . . . . . . . . . . . . . . . . 38

3.3.4 Whole blood clot composition . . . . . . . . . . . . . . 38

3.3.5 Sample preparation . . . . . . . . . . . . . . . . . . . . 39

3.3.6 Summary of Experiments _ . . . . . . . . . . . . . . . . 41

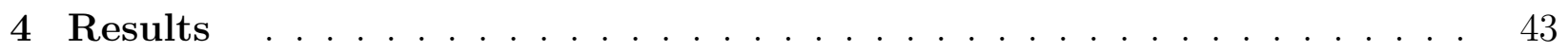

4.1 Human Blood Plasma . . . . . . . . . . . . . . . . . . . . . 43

4.1 .1 Prior to Well-Cap . . . . . . . . . . . . . . . . . . 44

4.1 .2 Using the Well-Cap . . . . . . . . . . . . . . . . . . . 44

4.2 Murine whole blood . . . . . . . . . . . . . . . . . . 45

4.2 .1 Acoustic Velocity . . . . . . . . . . . . . . . . . . . . . 48

$4.2 .2 \quad$ M-mode . . . . . . . . . . . . . . . . . . . . . 50

4.2 .3 Reflection spectra $\ldots \ldots \ldots \ldots \ldots$

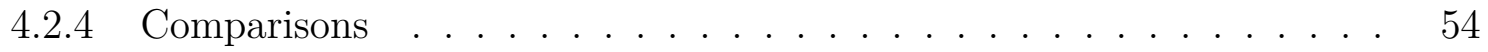

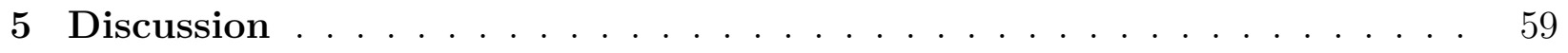

5.1 Human Blood Plasma . . . . . . . . . . . . . . . . . . . . 59

5.1 .1 Before the well cap addition . . . . . . . . . . . . . . . . 59 
5.1 .2 After the well cap addition . . . . . . . . . . . . . . 60

5.1 .3 Comparison of results . . . . . . . . . . . . . . . . 61

5.2 Murine whole blood . . . . . . . . . . . . . . . . . 61

5.2 .1 Acoustic velocity . . . . . . . . . . . . . . . . . 62

$5.2 .2 \quad$ M-mode . . . . . . . . . . . . . . . . . . . . . . 64

5.2 .3 Reflection spectra . . . . . . . . . . . . . . . . . 68

5.2 .4 Comparisons . . . . . . . . . . . . . . . . . . . . . 72

6 Summary and Conclusions _. . . . . . . . . . . . . . . . . . 74

\section{Appendices}

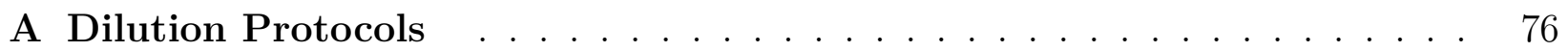

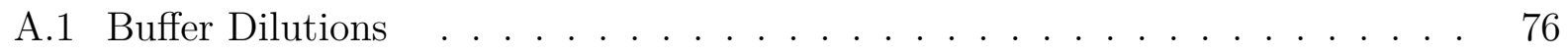

A.2 Clotting Factor and Fibrinolytic Enzyme Dilutions _ . . . . . . . . 77

B System Refinement Experiments _. . . . . . . . . . . . . . . . . . 79

B.1 Meniscus . . . . . . . . . . . . . . . . . . . . . . . . . 79

B.2 Acquisition Automation . . . . . . . . . . . . . . . 80

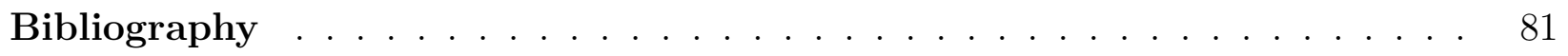




\section{List of Tables}

3.1 Transducer Specifications . . . . . . . . . . . . . . . . . . . . . . . . . 29

3.2 Number of replicates for each sample type in plasma and whole blood . . . . 42 


\section{List of Figures}

2.1 Longitudinal wave . . . . . . . . . . . . . . . . . . . 7

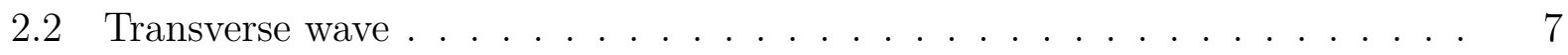

2.3 Artery wall . . . . . . . . . . . . . . . . . . . . . . . 14

2.4 Platelets . . . . . . . . . . . . . . . . . . . . . . . . 15

2.5 Coagulation cascade model . . . . . . . . . . . . . . . 16

2.6 Blood clots imaged using electron microscopy _ . . . . . . . . . . . 17

2.7 Reported relative ultrasound scatter intensity in coagulating human blood . 19

2.8 Reported integrated backscatter coefficient in coagulating human blood . . . 21

2.9 Reported changes in acoustic velocity for coagulating human and rat blood . 22

2.10 Reported spectral changes in coagulating human blood . . . . . . . . . . 24

3.1 Conventional SASAM setup . . . . . . . . . . . . . . . 27

3.2 Modified SASAM system . . . . . . . . . . . . . . . . . 28

3.3 Image of $80 \mathrm{MHz}$ transducer . . . . . . . . . . . . . . . . . . . . . . 29

3.4 Signal and power spectrum of $80 \mathrm{MHz}$ transducer . . . . . . . . . . 30

3.5 Schematic of acquisition setup and corresponding representative RF line . . 31

3.6 Schematic of acquisition setup with the well cap . . . . . . . . . . 35

3.7 Images of coagulated and uncoagulated blood samples . . . . . . . . . . . 41

4.1 B-Mode images of plasma samples with and without well cap . . . . . . . . 44

4.2 Measured change in arrival time in plasma samples without well cap . . . . . 45

4.3 Measured change in sound speed in plasma samples with well cap . . . . . . 46

4.4 Measured change in arrival time in plasma samples with well cap . . . . . . 46

4.5 Reflected frequency spectra in plasma samples with well cap . . . . . . . 47

4.6 B-Mode images of blood samples with and without clot retraction . . . . . 4 48

4.7 Measured intra-sample variation in sound speed in murine blood . . . . . . . 49

4.8 Measured inter-sample variation in sound speed in murine blood . . . . . . 49

4.9 Measured sound speed in control and coagulating murine blood sample . . . 50

4.10 M-mode images of murine blood with and without coagulation . . . . . . 51

4.11 M-mode images showing variability of clot onset in murine blood . . . . . . 51 
4.12 M-mode images showing variability of clot microstructures in murine blood . 52

4.13 Reflected frequency spectra of murine blood with and without coagulation . 53

4.14 Reflected frequency spectra showing variability of clot onset in murine blood 53

4.15 Reflected frequency spectra showing variability of clot microstructures in murine blood . . . . . . . . . . . . . . . . . 54

4.16 Comparison of reflected frequency spectra, M-mode images and speed of sound of murine blood with and without coagulation . . . . . . . . . 55

4.17 Comparison of reflected frequency spectra, M-mode images and speed of sound showing variability of clot onset in murine blood . . . . . . . . . 56

4.18 Comparison of reflected frequency spectra, M-mode images and speed of sound showing variability of clot microstructures in murine blood $(1 / 2) \ldots \ldots$

4.19 Comparison of reflected frequency spectra, M-mode images and speed of sound showing variability of clot microstructures in murine blood $(2 / 2) \ldots \ldots .58$

5.1 Net acoustic velocity change across mouse strains . . . . . . . . . . . 63

5.2 Variation of clot onset times across mouse strains . . . . . . . . . . 66 


\section{Chapter 1}

\section{Introduction}

\subsection{Hemostasis and Disease}

Hemostasis is the set of mechanisms present in humans that minimize blood loss and initiate the appropriate repair mechanisms following an injury [41, 67]. This system integrates a complex interplay of protein activations and induced cellular response near the site of the injury. The localized coagulant response to prevent blood loss must be simultaneously balanced by anti-coagulant and fibrinolytic pathways to maintain proper blood flow and prevent the formation of thrombi.

When these pathways become disrupted due to medication, injury, or disease, the excessive bleeding or undesirable clot formation that result can lead to morbidity or mortality. The most apparent instances of hemostatic disruption involve bleeding or clotting disorders (e.g. hemophilia and thrombophilia respectively), where genetic or acquired deficiencies in hemostatic protein levels prevent the healthy function of hemostatic mechanisms [9, 46, 77]. Hemostatic disruption is also relevant in the context of trauma or surgery, where significant blood loss can result in insufficient hemostatic protein levels that must be replenished through plasma or whole blood transfusions [27].

Furthermore, a large number of links have been postulated between hemostatic proteins and a wide range of diseases outside of blood disorders, including liver disease [7], cardiovascular disease [87], and diseases involving significant inflammatory responses, such as arthritis [24]. Tests to assess hemostatic disruptions are therefore of significant interest in the medical field, and are a focus of continuing research [58].

\section{Liver disease}

Most key proteins required for hemostasis are synthesized in the liver. Consequently, liver disease, whether acute or chronic, can lead to the development of hemostatic abnormalities in humans that increase the risk of bleeding and thrombosis [7, 25, 37, 47]. 


\section{Cardiovascular and circulatory disease}

Cardiovascular diseases are one of the leading causes of morbidity and mortality among Canadians [71, 72] and worldwide [94]. While there have been significant therapeutic advancements for many cardiovascular ailments, accurate risk assessment is still lacking [58]. As deviations from healthy hemostatic processes have been strongly linked to a range of cardiovascular diseases, including increased thrombin levels and venous thromboemboli [6, 23, 87], acute coronary syndromes [65, 81, 82], and stroke [17], hemostatic assessments are highly relevant to assessing and predicting cardiovascular risks.

\section{Inflammation}

Increasing evidence has suggested a strong link between hemostatic factors and the regulation of inflammatory response [24]. Specific molecular mechanisms have been identified that link a range of inflammatory diseases to fibrinogen and other important hemostatic proteins. This has been reported in the inflammatory responses associated with vascular wall disease [49, 62, 88], stroke [20, 30], multiple sclerosis [1], and rheumatoid arthritis [32]. Davalos et al. provide a comprehensive review of other disease where the inflammatory response has been linked to hemostatic factors [24].

\subsection{Coagulation Tests}

Crude assessments of coagulation are said to date back to at least the time of Hippocrates ( $400 \mathrm{BCE}$ ), with some reporting even earlier assessments by the somewhat mythical Yellow Emperor of China, known as Huangdi, who is thought to have lived over 5000 years ago [73]. These rudimentary tests involved assessing the duration of blood flow following a puncture wound. More reliable and diverse methods of testing components pertinent to coagulation have since been developed, many of which are used routinely in patient care.

\subsubsection{Routine coagulation tests}

Routine coagulation tests rely on similar principles to the rudimentary bleeding tests of Hippocrates, in that they evaluate the amount of time required for the onset of coagulation. They were developed with the intent of monitoring the impact of exogenous anticoagulant therapies prescribed for various illnesses. The most common are known as the prothrombin time (PT) and the activated partial thromboplastin time (aPTT) [58]. Both tests are performed on platelet poor plasma, which is the liquid that remains when all cells are removed from a citrated whole-blood sample through centrifugation. In both cases, an activating 
agent is added to initiate clot formation, and the amount of time required for coagulation to occur is measured in vitro using optical changes in the sample that occur due to coagulation. With major advancements in our understanding of the coagulation process, the effectiveness of these tests as anticoagulant monitors has been brought into question [4, 48]. Namely, their description of the coagulation process via a single measure (onset time) determined in an artificial environment is an oversimplification that limits their ability to predict the risk of bleeding or thrombosis [58].

\subsubsection{Global coagulation tests}

While routine coagulation tests such as PT or aPTT are still used for hemostatic assessment, they have been shown to have little predictive value for hyper or hypocoagulable states (which, respectively, can lead to undesirable clot formation or uncontrolled bleeding) [7, 37, 58]. Conversely, evidence is emerging that global coagulation tests show promise in risk assessment for a range of diseases [58]. Global coagulation tests are a general class of monitoring techniques that aim to provide a more comprehensive picture of potential hemostatic deficiencies than routine coagulation tests by acquiring kinetic data throughout the coagulation process.

\section{Thromboelastography}

Thromboelastography assesses changes in the viscoelastic properties of whole blood as it undergoes coagulation. Presently, two common commercial devices exist: TEG ${ }^{\circledR}$ and ROTEM $^{\circledR}$. These two devices have slightly different designs and, as a result, slightly different metrics used to assess changes in the sample. However, the physical principle is the same. Both involve a 'cup' into which a whole blood sample is deposited and a pin which is immersed into the sample. In TEG ${ }^{\circledR}$, the cup is oscillated while the pin remains stationary, while in ROTEM ${ }^{\circledR}$ the pin oscillates while the cup remains stationary. As blood coagulates, the underlying structure of the clot is formed. The formation of this structure creates a coupling between the components of the system, in which motion of the oscillating component is propagated through the sample, thereby inducing motion in the stationary component. The force felt by the stationary unit (reflected in the degree motion that it experiences) will depend on the elasticity and strength of the developing clot. This information is recorded throughout the clot's formation.

Thromboelastography is useful in point-of-care medicine, where it is used to assess the coagulopathy of patients prior to or during surgeries [10]. Furthermore, a range of studies have suggested links between thromboelastographic parameters and prognosis, including 
increased mortality risk in acute or chronic liver disease [7].

\section{Calibrated automated thrombogram}

The calibrated automated thrombogram (CAT) is an in vitro assessment of the time dynamics of thrombin content within the sample. Using a fluorogenic thrombin substrate, the rate of generation and overall quantity of thrombin is measured as a function of time throughout clot formation [40]. As this test relies on fluoresence, it must be performed in plasma instead of whole blood. However, the kinetic information provided can be valuable. Indeed, the CAT methodology has shown promise in identifying the risk of ischemic stroke $[17,59]$ and, according to some studies, coronary artery calcification [11, 86, 89]. In addition, it has recently been suggested that CAT can be used as a standardized test for hemophilia [22]. However, many of the studies have shown conflicting results and the specific nature of the link is still unclear [58].

\section{Overall hemostatic potential}

The overall hemostatic potential (OHP) is a laboratory test that assesses platelet activity, blood coagulation, and fibrinolysis in plasma [39]. In OHP, blood plasma is combined with thrombin and tissue plasminogen activator (t-PA), which initiate clot formation and lysis, respectively. A spectrophotometer is used to evaluate the optical transmission properties of the sample. As these properties are altered during plasma coagulation, the absorbance curves generated by the spectrophotometer contain information about the concentrations of coagulant factors and platelets within the plasma sample [2].

Small scale studies (of $<200$ patients) have indicated the ability of OHP to detect increased fibrin generation and reduced fibrinolysis in hypercoagulable patients [21], as well as impaired fibrinolysis in patients with coronary artery disease [38, 75].

\section{Limitations of global coagulation tests}

Despite the predictive and prognostic promise shown by global coagulation tests for a range of diseases, there are limits to the clinical applicability of current methodologies. For example, thromboelastography and CAT focus solely on the coagulation cascade. Given the complex interplay between coagulant, anticoagulant and fibrinolytic pathways, valuable information is lost when the latter two pathways are not considered. Additionally, CAT and OHP both rely on optical transmittance, which necessitates the use of plasma rather than whole blood, as light is unable to pass through the sample in the presence of blood cells. Thus, a technique 
that is sensitive to the three major hemostatic pathways and that could be used to investigate whole blood is desirable.

\subsection{Hemostasis and ultrasound}

Ultrasound (US) systems have long been used as diagnostic tools in the hospital due to their limited cost, portability and accessibility. With respect to its application to the study of blood and the hemostatic system, US is particularly effective at monitoring potential irregularities in blood flow and has been used for diagnosing deep vein thrombosis (DVT) [35], hypertension [33], and aortic obstruction or aneurysm [29]. US has also been used for therapeutic purposes, where low intensity US combined with a thrombolytic agent aids in accelerated thrombolysis for DVT [31].

Outside of the clinic, US is currently being explored as a way to monitor in vitro blood coagulation [44]. Previous work has shown that several acoustic parameters, including backscatter, speed of sound, and attenuation, are sensitive to changes that occur during coagulation [66]. However, these studies have been performed on systems with poor spatial resolution relative to the size of red blood cells (US frequencies $<45 \mathrm{MHz}$ ) and have focused exclusively on clot formation rather than complete hemostasis. Additionally, the choice of acoustic parameters measured in the literature is highly variable and inconsistencies amongst the reported results are prevalent.

\subsection{Hypothesis and outline of the thesis}

This thesis proposes the use of high-frequency ultrasound (HFUS) as a global coagulation test for the evaluation of the full hemostatic process in whole blood. The work presented in this thesis had two major goals: (i) to develop an ultrasonic measurement system analogous to the OHP, and (ii) to provide proof-of-principle data. The system development focused on providing the ability to assess small whole blood volumes $(<100 \mu \mathrm{L})$ throughout the hemostatic process for US frequencies ranging from 25-95 MHz. Whole murine blood samples were used to assess the system's sensitivity to the kinetics of clot formation.

Chapter 2 contains a summary of relevant US theory, information on blood coagulation, and a brief overview of the application of US to the study of blood coagulation. Details of the system development and methodology used in this work are presented in Chapter 3. The results are given in Chapter 4 and discussed in Chapter 5. Finally, Chapter 6 provides the major conclusions of this thesis and highlights potential areas of future work. 


\section{Chapter 2}

\section{Background and Theory}

\section{$2.1 \quad$ Fundamentals of Ultrasound}

\subsubsection{Acoustic waves}

US imaging involves the generation of acoustic (mechanical) waves in tissue. The way in which the wave propagates will depend on the type of material, as well as its homogeneity. If the wave is recorded as it leaves the sample, insights about the structure and properties of the tissue can be understood based on how the waveform was altered from its initial transmission.

There are two general waveforms encountered in US imaging: (i) longitudinal waves, and (ii) transverse waves. Longitudinal, or compressional, waves effect motion in the medium along the direction of wave propagation. Particles in the medium are made to oscillate along the propagation axis, resulting in regions of compression (increased physical density) and rarefaction (decreased physical density). This process can occur in both solid and liquid media and is the predominant signal type generated and received in most ultrasonic imaging systems. A visualization of longitudinal wave propagation is shown in Fig. 2.1.

The acoustic wave may also result in the oscillation of particles along an axis perpendicular to the direction of acoustic wave propagation. This is known as a transverse, or shear, wave (Fig. 2.2). Such waves minimally affect the physical density of the medium and cannot propagate in liquid media such as blood samples. 


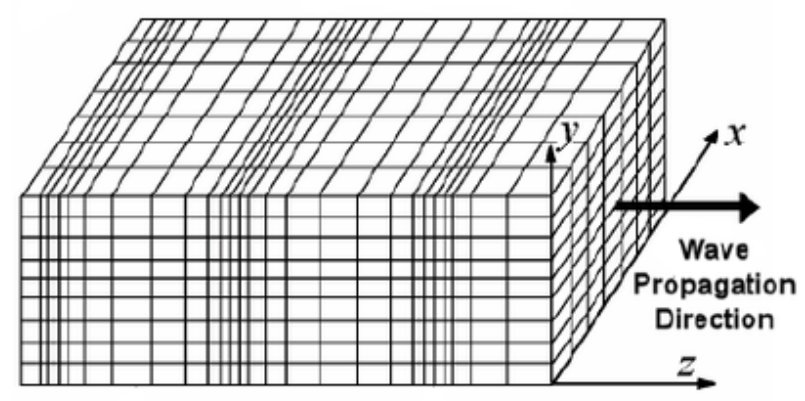

Figure 2.1: A schematic of a longitudinal wave. Longitudinal waves are one of the two general waveforms encountered in US imaging and result in changes in the medium along the direction of propagation. Image adapted from Cobbold [18].

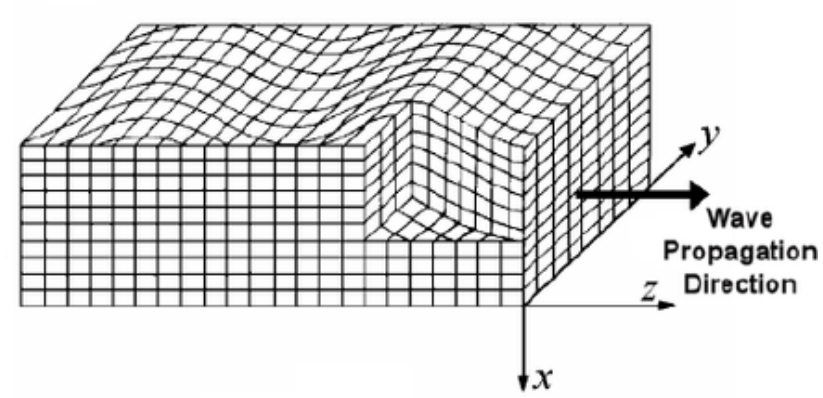

Figure 2.2: A schematic of a transverse wave. Transverse waves are one of the two general waveforms encountered in US imaging and result in changes in the medium perpendicular to the direction of propagation. Image adapted from Cobbold [18].

\subsubsection{Properties of acoustic media}

In this thesis, it is assumed that all acoustic wave propagation was adiabatic, meaning that the system under study did not undergo heat or mass exchange with its surroundings and thus entropy was approximately constant. This approximation is valid for the frequencies studied in this thesis $\left(<10^{8} \mathrm{~Hz}\right)[18]$.

\section{Compressibility and propagation speed}

The compressibility of a medium is defined as the amount that a volume element $d V$ will change when subjected to a pressure change $d p$. Under conditions of adiabatic propagation (constant entropy), compressibility $(\kappa)$ can be expressed mathematically as: 


$$
\kappa=-\left.\frac{1}{V_{o}} \frac{\partial V}{\partial p}\right|_{s}=\left.\frac{1}{\rho_{o}} \frac{\partial \rho}{\partial p}\right|_{s},
$$

where $\rho$ is the density of the medium (mass per unit volume), $s$ specifies conditions of constant entropy, and $\rho_{o}$ and $V_{o}$ denote the pressure and volume of the medium at equilibrium (i.e. in the absence of external pressures). Compressibility has units of inverse Pascals $\left(\mathrm{Pa}^{-1}\right)$, or equivalently in $\mathrm{m}^{2} \cdot \mathrm{N}^{-1}$. The speed with which the wave is able to propagate through a medium will depend upon the medium's compressibility. For a stiff medium (low compressibility), an acoustic wave will travel faster than in a more flexible medium (higher compressibility). The relationship between acoustic wave speed and adiabatic compressibility for liquid and gaseous media is given in Eq. 2.2.

$$
c_{o}=\frac{1}{\sqrt{\kappa \rho_{o}}}
$$

\section{Acoustic impedance}

In order to understand how waves will behave at an interface between two media, the concept of acoustic impedance must be introduced. The acoustic impedance, which can take on complex values in certain media, is often approximated using the characteristic acoustic impedance:

$$
Z_{o}=\rho_{o} c_{o}=\sqrt{\frac{\rho_{o}}{\kappa}}
$$

The SI unit for acoustic impedance is the Rayleigh (Rayl), which is equivalent to $1 \mathrm{~kg} \cdot \mathrm{s}^{-1}$. $\mathrm{m}^{-2}$.

When considering wave propagation at an interface, a portion of the wave is reflected while the rest is transmitted. The most common formalism to assess the proportion of wave reflected and the proportion transmitted is based on time averaged intensity ratios. The intensity reflection coefficient $\left(R_{I}\right)$ is defined as the ratio between the intensity of the incident wave to the intensity of the reflected wave. Assuming normal incidence, $R_{I}$ depends entirely on the characteristic impedance of the two materials: 


$$
R_{I}=\left[\frac{Z_{o, 2}-Z_{o, 1}}{Z_{o, 2}+Z_{o, 1}}\right]^{2}
$$

where the subscripts 1 and 2 the denote the two media at the interface, where 1 corresponds to the medium in which the incident wave is propagating. Similarly, the intensity transmission coefficient $\left(T_{I}\right)$ is the ratio of the transmitted wave intensity to the incident wave intensity and, under conditions of normal incidence, is expressed as:

$$
T_{I}=\frac{4 Z_{o, 2} Z_{o, 1}}{\left[Z_{o, 2}+Z_{o, 1}\right]^{2}}
$$

The two coefficients are related by $R_{I}+T_{I}=1$. Under the assumption of normal incidence Eqs. 2.4 and 2.5 can be applied to both solid and liquid media.

\section{Acoustic attenuation}

In addition to the intensity reduction at interfaces along the path of the acoustic wave, energy loss occurs due to effects such as absorption or scattering. Absorption is the conversion of the kinetic energy of the acoustic wave to heat and other forms of energy. Scattering is the redirection of portions of the wave, resulting in losses to the intensity along the direction of propagation of the main incident wave. In fact, reflection at an interface is a special case of scattering.

The attenuation coefficient $\alpha$ is used to quantify losses due to both absorption and scattering. It is generally presented on a logarithmic scale and is given in units of decibel $(\mathrm{dB})$ per centimetre. For an acoustic wave with an incident pressure $p(0)$ and a pressure $p(x)$ after travelling some distance $x$ through the attenuating medium, the attenuation coefficient is calculated as:

$$
\alpha=\frac{20}{x} \log _{10}\left(\frac{p(0)}{p(x)}\right)
$$

For most media, the attenuation coefficient has a frequency dependence, where high frequency acoustic waves are attenuated more strongly than low frequency waves. This can be expressed as: 


$$
\alpha=\alpha_{o} f^{n}
$$

where $n$ varies depending on the material. Water has a dependence of $n=2$, while in biological media such as tissues or blood, $n$ generally lies between 1 and 2 (with most values closer to 1).

\subsubsection{Transducers}

Ultrasonic transducers are the means by which acoustic waves are generated, focused and detected. They are typically made using one or more piezoelectric elements, which convert electrical impulses into mechanical waves, or vice versa. In most clinical systems, transducers are designed as an array of piezoelectric elements in order to produce two or even three dimension images [18]. For the purposes of this thesis, the main focus will be on single element transducers. Most systems, including the one used here, rely on pulse-echo measurements, where the transducer acts as both the transmitter and the receiver.

With single element transducers, acquired information corresponds to a single path through the sample. Focusing the acoustic wave can minimize dispersion as it travels through the medium, thereby resulting in larger echos (higher intensity) and signal-to-noise ratio (SNR) in the resulting RF line. Focusing also improves the lateral resolution of the RF line, making it desirable for techniques such as acoustic microscopy. The waveform in single element transducers can be focused using a lens. The acoustic lens is a part of the transducer and employs a concave shape to direct the wave towards its focus (point of maximum beam intensity). The focus and the lateral resolution at this point will depend on the geometry of the transducer. When a manufacturer produces a transducer, they provide information regarding the radius of the concave region (the aperture), and a parameter called the $\mathrm{f}$ number. The f-number is related to to the aperture radius $a$ and the radius of curvature of the transducer $z_{0}$ by:

$$
F \#=\frac{z_{0}}{2 a}
$$

If it is assumed that the wave front of the US acoustic wave can be approximated as rays emitted perpendicular to the lens' surface, the location of the focus would be equivalent to the radius of curvature of the lens. For this reason, $z_{0}$ is referred to as the geometric focus of the lens. However, diffraction of the wave front tends to bring the true focus closer to the 
transducer at a depth of $z<z_{0}$ [18]. The exact location will depend on the medium through which the wave propagates. Other parameters used to characterize the transducer include the aperture angle $\theta_{0}$ and the numerical aperture $N A$. These are defined in Equations 2.9 and 2.10 , respectively.

$$
\begin{aligned}
\theta_{0} & =\tan ^{-1}\left(\frac{a}{z_{0}}\right) \\
N A & =\sin \theta_{0}
\end{aligned}
$$

In acoustics, the focus doesn't correspond to a unique point but rather to a region wherein the beam reaches its maximum intensity. This region is typically defined in terms of the so-called $3 \mathrm{~dB}$ radius. Under this definition, all regions contained within the defined volume are within $3 \mathrm{~dB}$ of the maximum beam intensity. In general, the $3 \mathrm{~dB}$ radius provides a good estimate for lateral resolution [50]. The $3 \mathrm{~dB}$ radius is expressed mathematically as:

$$
d_{r}(3 \mathrm{~dB})=\frac{0.51 \lambda}{N A}
$$

where $\lambda=c_{o} / f$ is the wavelength of the US wave that depends on the speed of sound in the medium $c_{o}$ and the frequency of the transducer $f$.

The axial resolution is defined as the minimum distance between two scatterers along the direction of wave propagation required for them to be uniquely identifiable.

$$
d_{a}=\frac{c_{o} \cdot T}{2}
$$

where $T$ is the temporal length of the pulse.

In imaging, the transducer does not emit acoustic waves at a single frequency, but is rather emits a distribution of frequencies, centred at the central frequency of the transducer. The bandwidth describes spread of the frequency distribution about the central frequency. The pulse length is approximately inversely proportional to the bandwidth.

\subsubsection{Imaging modalities}

In pulse-echo systems, a short pulse is emitted by the transducer. This pulse travels through the material and portions of it are scattered or reflected at impedance discontinuities (interfaces). The echos received by the transducer are recorded in the form of a radio frequency 
line, or RF line, which conveys time dependant information regarding the pressure of the received signal. The delay time corresponding to a particular echo is related to the distance the wave travelled $d$ and the speed of sound in the medium:

$$
t_{d}=\frac{2 d}{c_{o}}
$$

If $c_{o}$ is known or, as is often the case in biological tissues, approximated as similar to that of water, then the RF line can provide spatial information regarding material boundaries or other sources of scatter.

\section{A-mode imaging}

In amplitude mode (A-mode) imaging, the time evolution of the amplitude of the pulseecho signal is recorded. Thus, it provides similar information to the RF line but does not differentiate between whether the pressure change is positive or negative relative to the equilibrium value of the medium under study.

\section{B-mode imaging}

Brightness mode (B-mode) imaging provides two dimensional information about the imaged sample. In multi-element transducers, B-mode images can be obtained from a single acquisition by using reconstruction algorithms to generate the image based on the delay times recorded at different locations in the array. For single element transducers, the image is constructed by combining a series of A-mode lines. These lines must be acquired sequentially by scanning the transducer across the object. The intensity of the A-mode lines can be translated to a grey or colour scale and, when combined, provide spatial information in both the axial (through delay time) and lateral (through the acquisition of multiple A-lines) directions.

\section{M-mode imaging}

Motion mode (M-mode) imaging relies on acquiring A-mode lines at regular time interval at a single transducer location. The time at which the A-mode line is acquired is referred to as the acquisition time. By combining all of the acquired A-lines into a two dimensional image with acquisition time on the x-axis, the motion of structures can be detected. Conventionally, the A-mode lines are acquired in quick succession to obtain information on rapidly moving 
structures such as the heart. However, M-mode imaging can also be useful in conveying changes on a slower time scale by decreasing the rate at which A-mode lines are acquired.

\subsection{Hemostasis}

\subsubsection{The hemostatic system ${ }^{1}$}

The hemostatic system has historically been organized into five components: (i) blood vessels, (ii) platelets, (iii) coagulant proteins, (iv) anti-coagulant proteins, and (v) fibrinolytic proteins. In addition to the five components mentioned above, increasing evidence suggests that red blood cells also play an active role in regulating the hemostatic response [92, 93].

\section{Blood vessels}

Fig. 2.3 shows the typical structure of an artery wall. The artery wall is composed of three distinct layers, separated by elastic membranes: (i) the tunica intima, (ii) the tunica media, and (iii) the tunica externa. The tunica intima is composed of a single layer of endothelial cells that line the innermost region of the vessel, backed by a layer of elastic tissue. The tunica media, is composed of smooth muscle cells, elastic tissue, and collagen (an important structural protein). The tunica externa, is mainly composed of collagen and provides structure to the vessel. The anatomy of a vein is similar, although it lacks the internal and external elastic membranes. Additionally, the tunica media is thinner in veins than in arteries and tends to have a larger proportion of collagen.

\footnotetext{
${ }^{1}$ The topics covered in this section follow the explanations presented by Pasi and Hiller [41, 67]
} 
a)

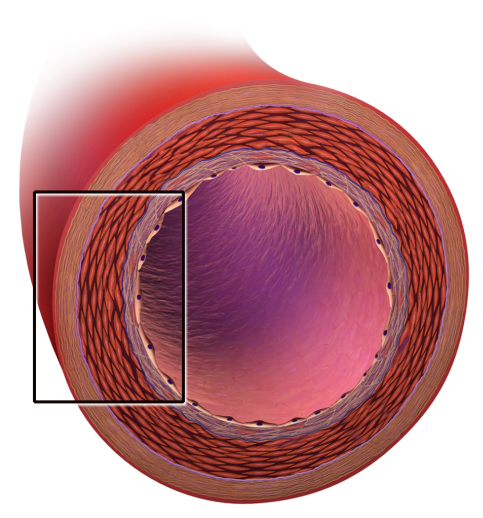

b)

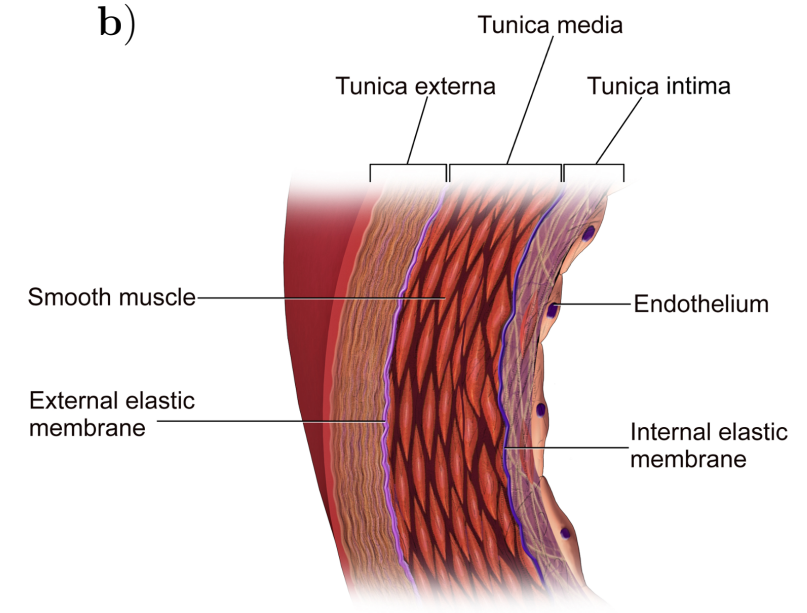

Figure 2.3: The structure of an artery wall. The boxed region indicated in a), is shown in b). Adapted from Blausen [8].

The endothelial cells are necessary for maintaining healthy blood flow. When no injury is present, this layer of cells releases a series of anti-coagulant and anti-platelet substances preventing undesirable coagulation, while tight cell junctions prevent blood loss [67]. When damage to the vessel occurs, pro-coagulant factors such as tissue factor are released from the cells, in order to trigger the coagulation pathway. Additionally, a damaged vessel can respond by undergoing vasoconstriction, which is the process that occurs when the smooth muscle cells in the tunica media contract. The contraction decreases the size of the damaged region of the vessel, thus minimizing blood loss. This is more effective in arteries (which have a thicker tunica media), however it can still occur to a limited to degree in veins [41].

\section{Platelets}

Platelets (cytoplasmic fragments without nuclei) are generated in the bone marrow and lungs. Also known as thrombocytes (from the Greek thrombos, meaning clot, and kytos, meaning cell), their main function is to prevent blood loss following a vascular injury. This is accomplished through the initiation of blood clot formation. In the absence of damage, platelets circulate freely in the blood. However, when the collagenous fibres of a vessel's subendothelial layers are exposed to blood due to injury, receptors on the platelets' surface will begin to attach themselves to the fibrous region in a process known as platelet adhesion. As platelet adhesion occurs, the platelets undergo significant morphological changes that drastically increase their surface area. A schematic of this process, known as platelet activation, is shown in Fig. 2.4. The increased surface area allows for the efficient release of additional pro-coagulant factors and signalling compounds which encourage platelet aggre- 
gation. This series of interactions results in the formation of a 'platelet plug', which acts as a temporary barrier against blood loss. The response is initiated by the tissue factor release from the endothelial cell, and results in the formation of a platelet plug. This known as primary hemostasis, and provides a temporary structure to prevent further blood loss until a more stable reinforcement is formed (secondary hemostasis).

a)

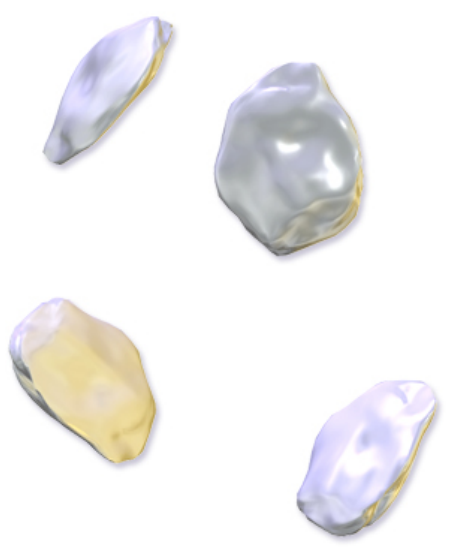

b)

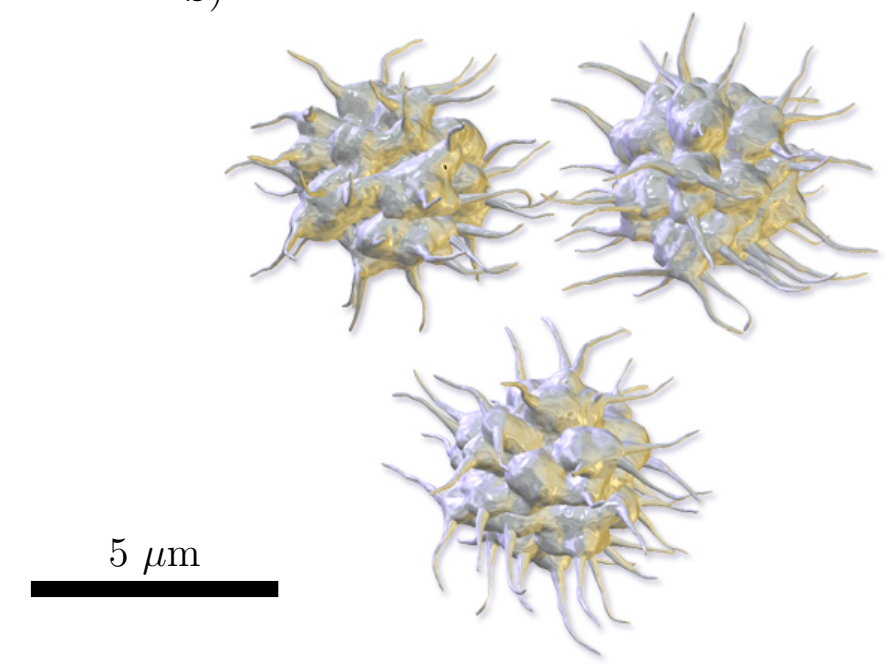

Figure 2.4: Platelets in their a) inactive and b) active morphologies. The scale bar shown is approximate. Adapted from Blausen [8].

\section{Coagulation factors}

Secondary hemostasis involves the formation of a stable fibrin clot to reinforce the platelet plug. The proteins necessary for fibrin production are always present in the blood; however, they are typically in an inactivated state. These proteins are known as clotting factors and are denoted by roman numerals. The active forms are denoted by the same roman numeral followed by an 'a'. Historically, fibrin clot formation was divided into three pathways: intrinsic, extrinsic, and common (Fig. 2.5). This simplified depiction of coagulation is known as the coagulation cascade, and was first hypothesized in 1964 [61]. 


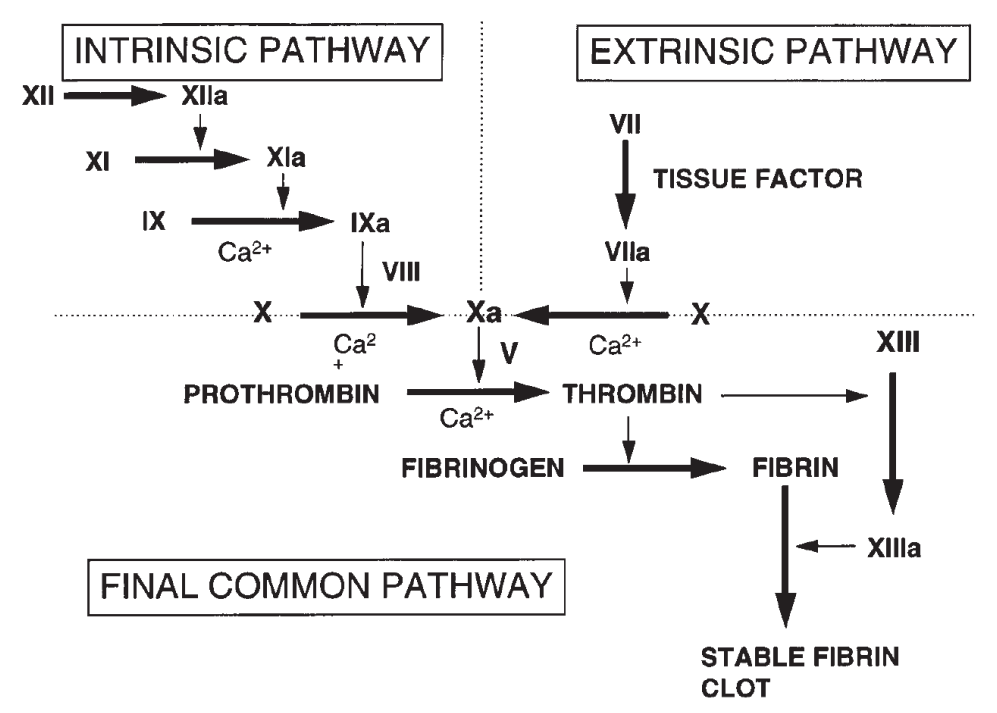

Figure 2.5: The simplified cascade model for fibrin clot formation. Adapted from Pasi [67].

The intrinsic pathway is initiated when (clotting) factor XII is activated by charged particles released by the aforementioned activated platelets to become factor XIIa. This activation can also occur in the presence of negatively charged surfaces, and is the reason that blood tends to coagulate when placed in a glass vial. Once the first factor in a pathway is activated, it in turn will activate the following protein in the cascade, and so on until the active form of fibrin is reached [61]. The extrinsic pathway is triggered by the release of tissue factor from damaged endothelial cells. Both the intrinsic and extrinsic pathways lead to the activation of the so-called common pathway. Ultimately, this series of activations results in large quantities of fibrin monomers, which quickly undergo polymerization into long protein chains. These chains are stabilized by factor XIIIa (also known as fibrin stabilizing factor) through cross-linking of the polymer chains. The fibrin polymers make up a stabilizing mesh that holds the clot together. Factor XIIIa can also cause the fibres to contract, resulting in a stiffer, denser clot structure. Electron microscopy images of the clot microstructure with varying concentrations of red blood cells (RBC) is shown in Fig. 2.6. 


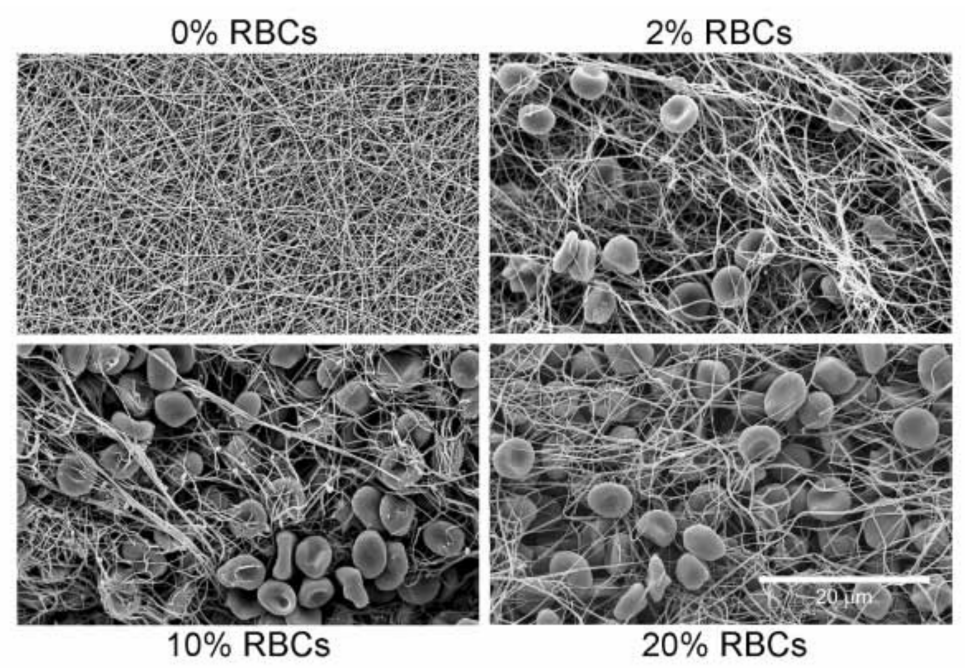

Figure 2.6: Blood clots formed with $0 \%, 2 \%, 10 \%$ and $20 \%$ RBC by volume. Images were acquired by scanning electron microscopy at 1000X magnification. Adapted from Gersh et al [34].

In vitro, the intrinsic and extrinsic pathways are often tested independently of one another in order to assess the function or abundance of certain clotting factors along each pathway. This approach has been useful in the diagnosis of bleeding disorders. However, at the time that the cascade hypothesis was proposed in 1964 [61], many aspects of hemostasis were still poorly understood. Important inhibitory pathways, and the multifaceted roles played by many of the enzymes were not considered and thus the cascade hypothesis does not not account for the full complexity of the processes that can occur in vivo.

\section{Anticoagulant pathways}

Anticoagulants disrupt the fibrin clot formation pathways by inhibiting the function of activated factors. One class of anticoagulants blocks the active sites of activated factors, thereby preventing them from initiating subsequent steps in the cascade. A second class of anticoagulants accomplishes the same result by simply breaking down the activated factors.

\section{Fibrinolysis}

Fibrinolysis (occasionally referred to as tertiary hemostasis) is the breakdown of the fibrin polymers that make up the fibrin clot. This process is induced to prevent the build up of clots larger than what is necessary to prevent blood loss. Thus, fibrinolysis ensures that there is reduced potential for the blockage of blood flow by stationary or mobile clot remnants. The polymer breakdown is primarily affected by an enzyme called plasmin, which cleaves fibrin into fibrin degradation products. Plasmin is the active form of plasminogen and can 
be activated by endogenous proteins known as plasminogen activators. Tissue plasminogen activator ( $t-P A)$ is released by endothelial cells when they are damaged but does not function effectively as a plasminogen activator until it has bound to fibrin. In other words, it is released at the time of the injury but does not cause polymer breakdown until the fibrin network has begun to establish itself. Another endogenous compound that activates plasminogen is urokinase plasminogen activator ( $\mathrm{u}-\mathrm{PA})$, which is most commonly activated by the presence of plasmin itself.

\section{Red blood cells}

It is well established that the flow of RBCs forces platelets towards the edges of the vessel, thereby allowing for more effective platelet adhesion; a critical stage in primary hemostasis [85]. Until recently, however, RBCs' involvement in coagulation has been considered passive, in that they become trapped in the fibrin mesh but do not contribute to any of the biochemical processes [26]. At present, an increasing body of evidence suggests a correlation between thrombin generation and the presence of RBCs [92]. While the mechanisms are still poorly understood, it is theorized that the $\mathrm{RBC}$ membrane can act as a pro-coagulant surface under certain conditions $[42,64,68]$. Furthermore, it has been suggested that they simultaneously play an anticoagulant role, suggesting their involvement as an important hemostatic regulator [92, 93]. As a result, evaluating hemostasis in whole blood (as opposed to plasma) is desirable as it provides a more representative picture of in vivo hemostasis by including the contribution of RBCs to clot formation, regulation, and breakdown.

\subsubsection{Hemostasis in Mice}

The hemostatic response is thought to be closely related among all mammals [60]. While the human hemostatic system is the most well studied, research has also been performed using animal models. Of particular interest to this thesis is the response observed in mice, which have previously been used for hemostatic experiments $[13,51]$. However, compared to humans, clotted mouse blood is highly resistant to lysis (i.e. clot breakdown). With t-PA as the studied lytic agent, they were found to be $>30$-fold more resistant to lysis than human

clots and with u-PA this discrepancy was even more pronounced [56]. Thus, care should be taken when extrapolating results to human models [57]. 


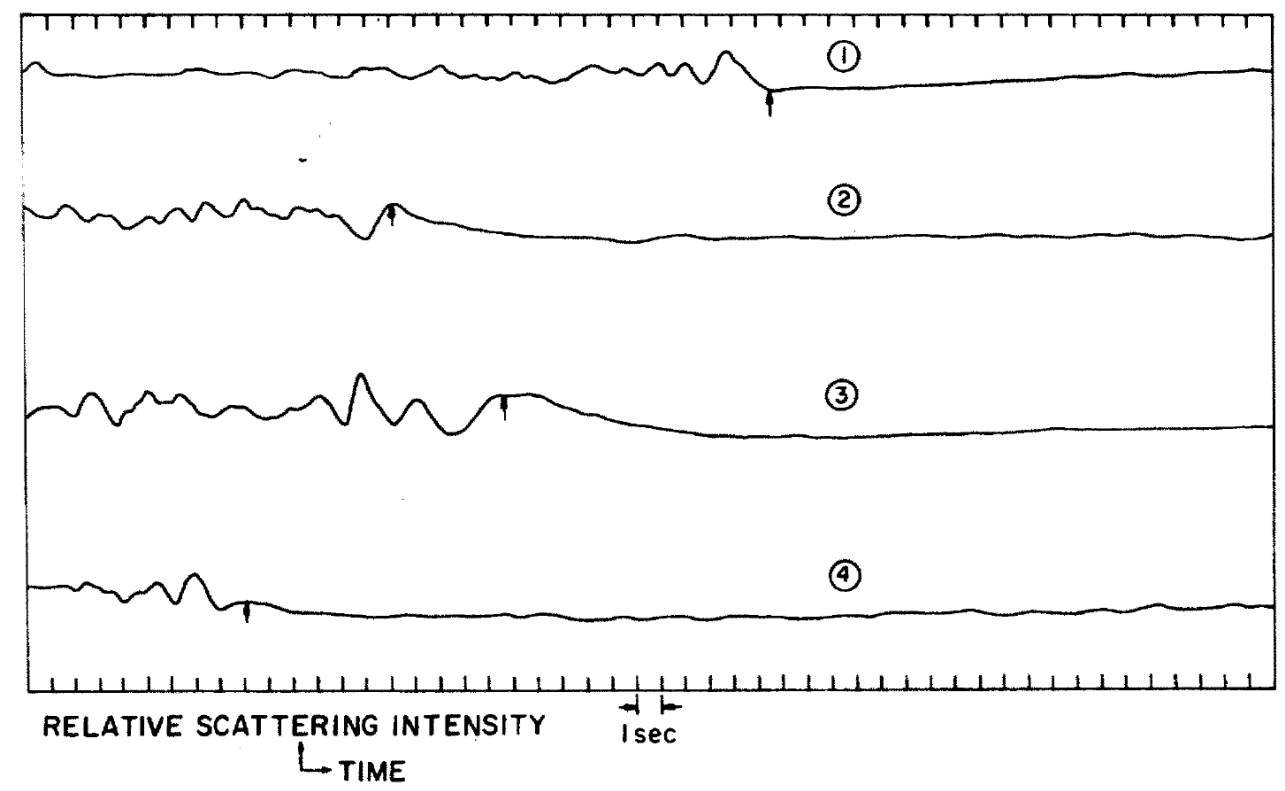

Figure 2.7: Relative US scattering intensity from various dilutions of whole blood samples following the addition of thrombin to initiate clot formation. From top to bottom, the samples contain $10 \%, 25 \%, 20 \%$ and $35 \%$ blood diluted in a saline solution. The arrows indicate the onset of clot formation for each sample. Adapted from Shung et al. [80].

\subsection{Ultrasound and blood coagulation}

The use of US for hematological studies was first proposed in 1975 by Shung et al. [80], who reported a proof of principle experiment that demonstrated the sensitivity of an US system to differentiate between the backscatter signal from liquid and clotted blood. It was found that the time of clot onset corresponded to the stabilization of the backscatter intensity, which otherwise fluctuated as a result of RBC motion. The measured time of onset following initiation of clot formation using thrombin was dependent on the amount of saline used to dilute the blood sample [80]. A delayed time of onset in the dilute samples indicated an association between clot formation time and the concentration of fibrin in the sample [80],. Their published results are shown in Fig. 2.7. The early experiments of Shung et al. [80], led to the development of a variety of methods to study the coagulation of whole blood and blood plasma using US. These include measurements of: (i) backscatter, (ii) speed of sound, (iii) attenuation, and (iv) scatterer motion using M-mode imaging.

To date, there has been limited translation of these technologies to hematological laboratories and hospitals. However, international research has highlighted the technique's promise, and will be described in the coming sections. 


\subsubsection{Backscatter measurements}

The backscatter coefficient $\sigma_{b}$ represents the amount of acoustic energy backscattered from a particular region of interest (ROI) within the sample. In the context of coagulation monitoring, a similar parameter has been used known as the integrated backscatter coefficient (IBC), which is defined in Eq. 2.14.

$$
\mathrm{IBC}=\int_{f_{\min }}^{f_{\max }} \frac{\sigma_{b}(f)}{f_{\max }-f_{\min }} d f
$$

where $f_{\min }$ and $f_{\max }$ are the minimum and maximum frequencies, respectively, and which correspond to values chosen within the bandwidth of the transducer.

In experiments performed to measure IBC in coagulating blood, literature values for $f_{\min }$ and $f_{\max }$ are typically 10 and $30 \mathrm{MHz}$ respectively [15, 54, 55, 66]. Clot onset was initiated through the addition of $\sim 15 \mathrm{mM}$ calcium chloride, which results in clot formation at approximately 40 minutes in human blood $[15,54,55,66]$. The IBC showed an increase for the first several minutes of the acquisition, which was attributed to RBC aggregation. Following this initial increase, the IBC reached a plateau. With the onset of clot formation (which generally occurred between 20 and 40 minutes), the results were highly variable $[15,54,55,66]$. Most of this variability was attributed to in-frame clot retraction (referred to in some papers as serum expulsion). This refers to the process whereby RBCs become trapped in the contracting fibrin mesh and, on the occasions that they pull away from the edges of the sample holder, leave regions filled with serum. Serum, which is essentially plasma without any coagulant proteins, contains minimal scatterers and therefore a very small backscatter coefficient. Thus, if a region of serum becomes visible within the ROI for the backscatter calculations it will negatively impact the results. An example curve for IBC data collected from four volunteers is shown in Fig. 2.8. The grey region indicates values that have been excluded from the analysis because they occurred after the in-frame clot retraction.

Since no conclusions can be drawn from the regions that contain the serum, the main IBC characteristic extracted by the authors pertained to the changes observed in the first several minutes due to RBC aggregation $[15,54,55,66]$.

\subsubsection{Speed of sound}

Sound speed measurements for biological media are of significant interest in US research, as they can affect metrics used for diagnostics and treatment planning [36]. As such, sound 


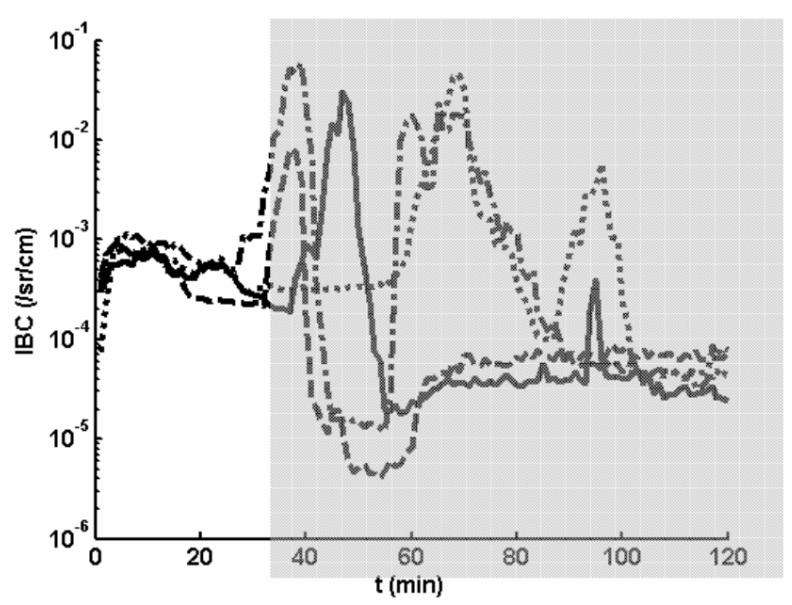

Figure 2.8: The IBC is shown as measured in coagulating blood samples from four volunteers for a frequency range of 10-30 MHz. Adapted from Libgot et al. [54].

speed in whole blood and blood plasma are well documented. On the other hand, changes that occur during coagulation have only been performed by a small number of researchers.

\section{Whole blood}

In whole blood, sound speed depends mainly on temperature and on hematocrit levels (HCT), which describe the percentage of red blood cells by volume in a sample of whole. For women, average HCT levels are 40.7\% for women [14], while for men they are 45.2\% [91]. A linear relationship between hematocrit level and speed of sound has been observed [5, 12, 28, 79], where the most commonly used relationship (for blood samples at $37^{\circ}$ ) is described in Eq. 2.15.

$$
c_{\text {blood }}=1541.82+0.98 \cdot H C T
$$

For average HCT levels, sound speed ranges from $\sim 1582-1586 \mathrm{~m} / \mathrm{s}$.

It has been reported that the sedimentation of RBCs (or settling of RBC due to gravity) affects the acoustic properties of blood [74]. Increases in the sound speed due to sedimentation were approximately linear with time and resulted in an overall change on the order of $10 \mathrm{~m} / \mathrm{s}$ after a period of 90 minutes $[15,16,53,66]$.

As blood coagulates, fibrin polymerizes and RBCs become trapped in the newly formed fibrin mesh (Fig. 2.6). An example of the changes observed in two volunteers following the re-calcification of a citrated blood sample is shown for both human and rat samples in Fig. 2.9. An increase in the acoustic velocity of between 30 and $40 \mathrm{~m} / \mathrm{s}$ was observed. The 

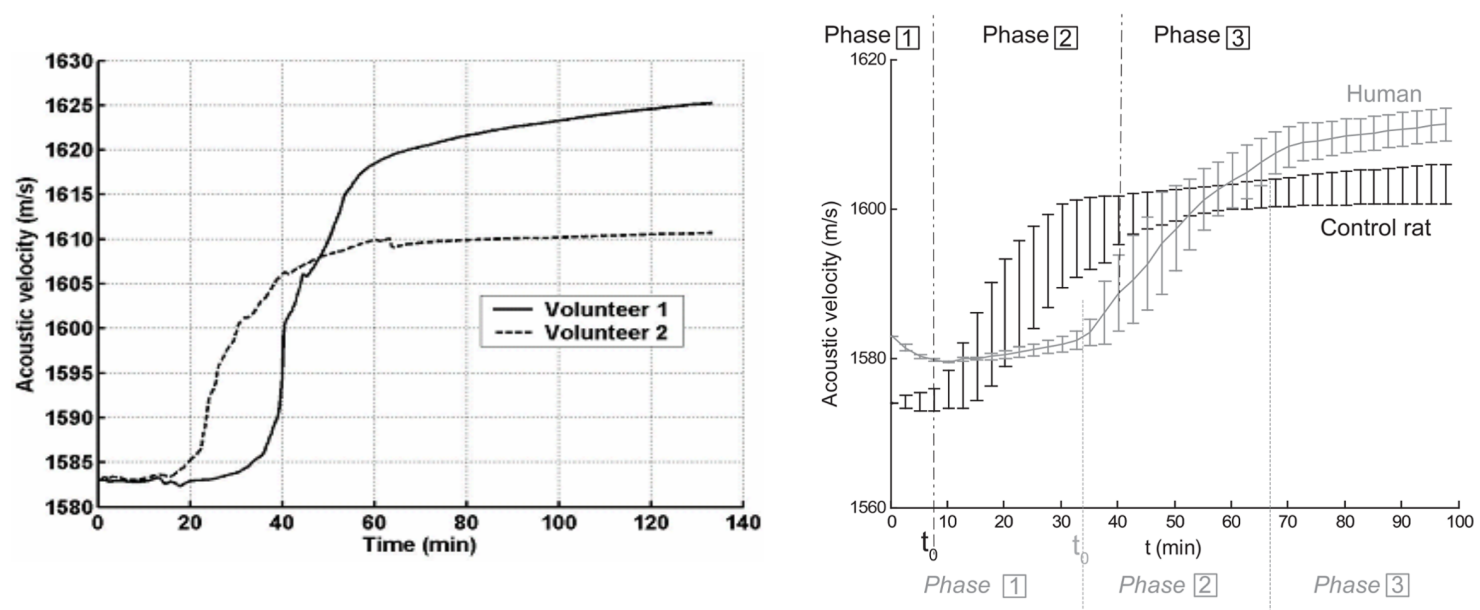

Figure 2.9: Temporal changes in the US velocity are shown for (A) two volunteers, as reported by Ossant et al [66], and (B) a human and a rat sample as reported by the same group [16].

phases indicated in Fig. 2.9(b) were thought to correspond to three stages of clot formation: (1) a liquid phase prior to formation, (2) the polymerization of the fibrin mesh (gelation), and (3) the stable clot structure. However, in a later paper by the same authors, the increase in acoustic velocity was instead attributed to the stiffening of the clot, while the gelation was thought to happen prior to this [70]. It should be noted, however, that the underlying causes of changes in the acoustic velocity have not yet undergone validation [70].

The aforementioned trends are consistent with those reported by another group, who performed similar experiments using human blood [84]. Older results published from experiments using porcine blood showed much slower and less significant changes [45].

\section{Blood plasma}

In blood plasma, the HCT value is virtually zero (as the red blood cells have been removed) and variation occurs mainly due to plasma protein concentration. Note that this also affects the sound speed in whole blood; however, relative to HCT contributions, the changes in speed of sound are minimal. At $37^{\circ} \mathrm{C}$, the relationship between sound speed and plasma protein crystal concentration $P$ is $2.16[3,28,43]$ :

$$
c_{\text {plasma }}=1523.9+3.36 \cdot P
$$

where $P$ is in $\mathrm{g} \mathrm{dL}^{-1}$. $P$ typically falls in the range of $7 \pm 1 \mathrm{~g} \mathrm{dL}^{-1}$, resulting in plasma sound speeds of $\sim 1544-1551 \mathrm{~m} / \mathrm{s}$. This predicted range is in good agreement with experimental values reported by other groups [19, 69]. Plag et al. observed an approximately linear 
dependence of sound speed on temperature (in Celsius), with sound speeds of 1544-1553 m/s corresponding to temperatures of $33-38^{\circ} \mathrm{C}$ [69]. The protein concentration was not recorded but was assumed to lie within the normal range $\left(7 \pm 1 \mathrm{~g} \mathrm{dL}^{-1}\right)[69]$.

Experiments on coagulated plasma samples are less widely reported but it has been shown that the coagulation had no measurable impact on the acoustic velocity in the plasma samples [69].

\subsubsection{Attenuation and spectral intensity}

Previous studies on the effect of acoustic attenuation on US signal intensity have described the correlation using a parameter called the integrated attenuation coefficient (IAC). Using $\alpha(f)$ as the frequency dependent attenuation coefficient (Sec. 2.1.1), the IAC is defined as:

$$
\mathrm{IAC}=\int_{f_{\min }}^{f_{\max }} \frac{\alpha(f)}{f_{\max }-f_{\min }} d f
$$

\section{Whole blood}

The studies that reported IAC values integrated across frequency ranges of 8-22 $\mathrm{MHz}$ [15] and $15-45 \mathrm{MHz}$ [69]. For both ranges, an initial increase in the IAC was observed during phases that the authors attribute to RBC aggregation and the early stages of fibrin polymerization. This was followed by a decrease in the IAC, which was thought to correspond to the stabilization phase of the fibrin structure. In the fourth phase, they reported high variability, which was attributed to serum expulsion within the frame and clot movement caused by the mesh contraction.

The attenuation coefficient as defined in Eq. 2.6 was measured in two other experiments. Peak-to-peak signal amplitude from an echo off of the container's wall was used as a proxy for the final pressure $p(x)$ and was normalized to the signal amplitude of the same echo in a water sample for $p(0)$. When the attenuation coefficient is calculated in this way, it is referred to as the relative attenuation coefficient. These experiments were performed using central frequencies of $5 \mathrm{MHz}$ [90] and $10 \mathrm{MHz}$ [45]. In both cases, an increase in the attenuation coefficient was observed during what was considered to be clot formation. No other features were clearly identifiable from these experiments. While the IAC values are reported to have decreased as a result of clot formation [15, 69], the relative attenuation coefficient was found to increase $[45,90]$. Although both metrics are meant to provide information on the sample's attenuation, this discrepancy could be due to the fact that the methods of evaluation are not directly analogous (IAC vs. relative attenuation coefficient) as well as the difference in 

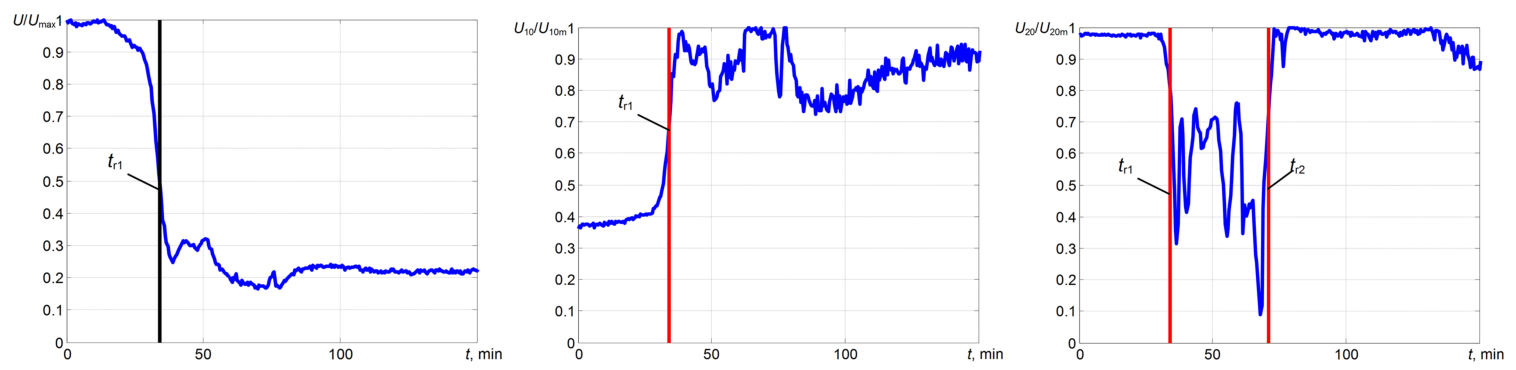

Figure 2.10: Spectral components of a reflected pulse from a strong reflector for sample that undergoes coagulation. From left to right, the normalized change in spectrum maximum of the signal, the normalized variation in the $10 \mathrm{MHz}$ component of the signal, and the normalized variation in the $20 \mathrm{MHz}$ component of the signal. Adapted from Tatarunas et al. [84].

frequency ranges considered. A recent paper reported strong frequency dependant changes in the signal intensity of a reflected US pulse that passed through a coagulating blood sample [84]. Normalized frequency data was acquired using a $20 \mathrm{MHz}$ transducer with a $20 \mathrm{MHz}$ bandwidth [84] and it was found that the trends seen during clot formation differed depending on the frequency [84] (Fig. 2.10). When looking at the normalized change in the signal maximum from the reflected pulse, a significant drop is observed at a time $t_{r 1}$, which is said to correspond to the start of the clot's contraction [84]. However, looking at the specific spectral components (Fig. 2.10), an increase in the intensity can be seen at $10 \mathrm{MHz}$, while a decrease can be seen at $20 \mathrm{MHz}$ for the same time point. Furthermore, the time of clot stabilization $\left(t_{r 2}\right)$ has been highlighted in the $20 \mathrm{MHz}$ normalization due to the significant changes observed at the higher frequency.

\section{Plasma}

A study reported the IAC in plasma for samples with and without thrombin. Recall that the addition of thrombin initiates coagulation. It was found that the amplitude of variation between the coagulating samples and the non-coagulating (without thrombin) samples was not large enough to be deemed statistically significant [69].

\subsubsection{M-mode imaging}

Two studies have reported on the use of M-mode imaging to monitor coagulation: Huang et al. [45] and Wang et al. [90]. Since M-mode provides visualization of motion within the frame, both studies observed that clot formation was visible through the immobilization of scatterers. The Huang et al. study observed a depth dependent onset time in one of the images acquired with a $10 \mathrm{MHz}$ transducer, with apparent variations on the order of $\sim 5$ 
minutes [45]. No explanations were discussed in the paper. In the Wang et al. study, the poor contrast of the images, which were acquired using a $5 \mathrm{MHz}$ transducer, made identification of onset time difficult [90].

\subsubsection{Context of work}

In this work, a HFUS system was developed to examine changes in the speed of sound, frequency dependant attenuation, and M-mode measurements during the coagulation process. These three parameters were compared, and for the first time, examined in a frequency range higher than $45 \mathrm{MHz}$ [69]. Using a central frequency of $80 \mathrm{MHz}$ achieved better backscatter strength and resolution for the M-mode images, and also produced measurements of changes in the frequency dependant attenuation at higher frequencies than have previously been observed in coagulating blood samples. Additionally, these metrics were measured at multiple locations in each sample, providing previously unreported insight into the heterogeneity of samples under $100 \mu \mathrm{L}$. 


\section{Chapter 3}

\section{Materials and Methods}

\subsection{Acoustic Acquisition System}

\subsubsection{Conventional use}

The Saarland Scanning Acoustic Microscope (SASAM, Kibero GMBH, Germany) was used as a base for the acoustic data acquisition. In its original set up, a single element transducer is connected to an adjustable arm and is directed downwards towards a mechanical stage. The sample is placed on the stage and can be viewed optically via an objective microscope that is focused on the sample from below (Fig. 3.1). The system can also be used for photoacoustic (PA) imaging, wherein laser light is focused through the objective onto an optically absorbing chromophore which undergoes thermoelastic expansion. The resulting mechanical waves produced in the sample can be detected by the transducer. This setup has many practical applications and has been used extensively for acoustic and photoacoustic microscopy. Since it has many applications for biological assessment, the entire structure shown in Fig. 3.1 is encased in a chamber that is kept at $37^{\circ} \mathrm{C}$, which is approximately the internal body temperature in humans.

In the context of this thesis, the main drawback of the conventional SASAM setup arises from the coupling requirements; mainly, the need to immerse the transducer directly into the sample. For coagulation monitoring, this would require thorough decontamination of the transducer. However, inter-sample biological contamination could still result. This is of particular concern when various coagulant or fibrinolytic enzymes are used that could affect the kinetics of control samples. As such, a new method of acquiring data was developed.

\subsubsection{System modifications}

While the objective microscope can provide useful guidance for transducer alignment in acoustic microscopy (where high spacial accuracy is required), a visual assessment of the transducer alignment was considered sufficient for these experiments due to the relative homogeneity of the samples that were analyzed. For ease of modification, a transducer stand was developed to fit into the objective stand such that the transducer was directed 


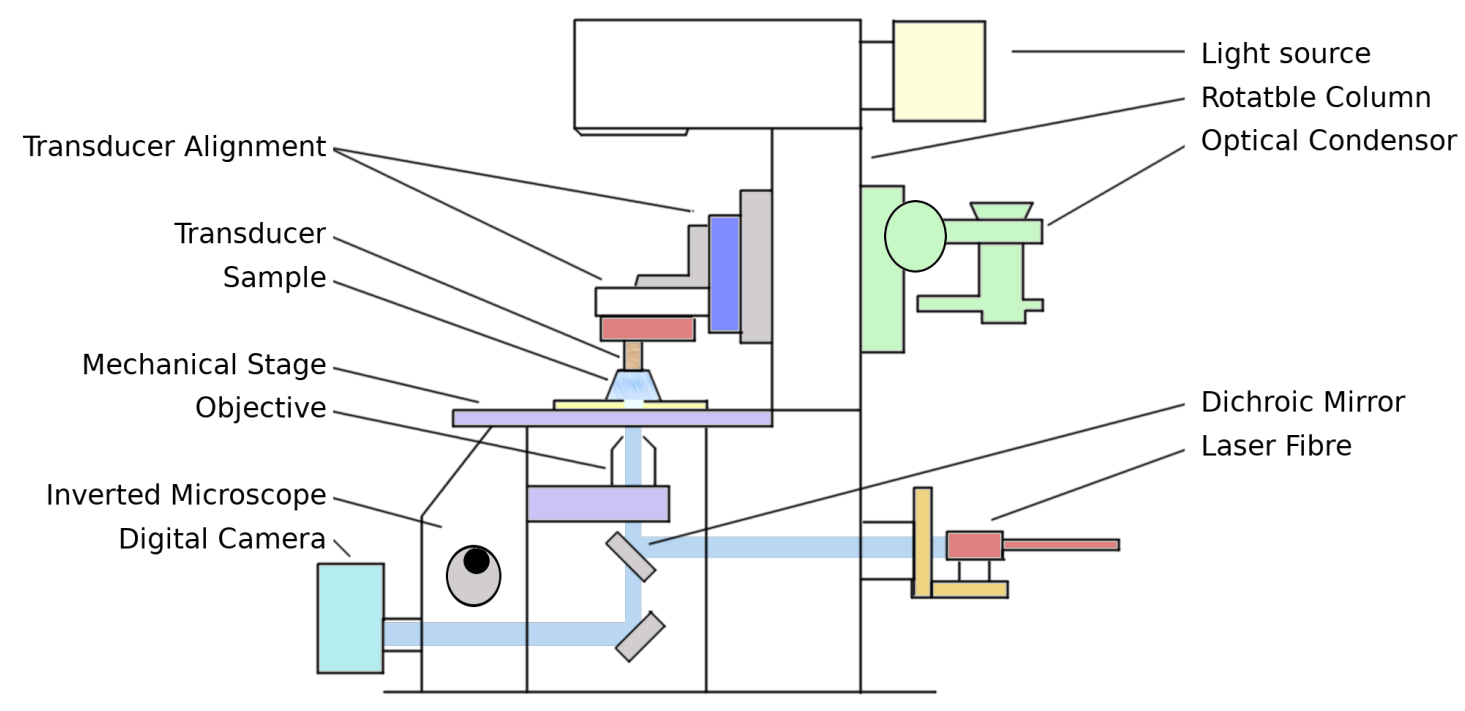

Figure 3.1: The SASAM in its original set up is shown. Figure based of off work by Rui et al. $[76]$.

upwards from below the sample (Fig. 3.2). The conventional sample holder was replaced by a 96-well microtitre plate. As the name implies, the plate contained 96 wells which were arranged in an $8 \times 12$ matrix. Each well had a working volume of $340 \mu \mathrm{L}$, with a diameter of $\sim 6.4 \mathrm{~mm}$. Measurements were acquired by coupling the transducer (which had a diameter of $\sim 7 \mathrm{~mm}$ ) to the bottom of a single well using a droplet of water. The coupling was maintained by the surface tension of the water droplet and was made possible by the flat surface of the $80 \mathrm{MHz}$ transducer (see Fig. 3.3).

The SASAM has two built in pulsers, designed for $400 \mathrm{MHz}$ and $1 \mathrm{GHz}$ transducers, respectively. Therefore, when the $80 \mathrm{MHz}$ transducer was used an external pulse generator was necessary. The $80 \mathrm{MHz}$ pulse generator (AVB2-C, Avtech Electrosystems Ltd., Canada) was connected by bypassing the $1 \mathrm{GHz}$ pulse generator.

\subsubsection{Acquisition settings}

Data acquisition was performed using the Acoustic Investigator, which is a custom MATLAB GUI that enables the user to control the acquisition settings as well as to visualize and save the data recorded by the device.

In the system developed here, there was a trade-off between the sampling frequency and the RF window size, as only a limited amount of data points could be stored per RF line. A $1 \mathrm{GHz}$ sampling frequency was chosen to allow for acquisition windows up to $8000 \mathrm{~ns}$. By recording echos received between 4000 and 12000 ns, all reflections from the sample were 


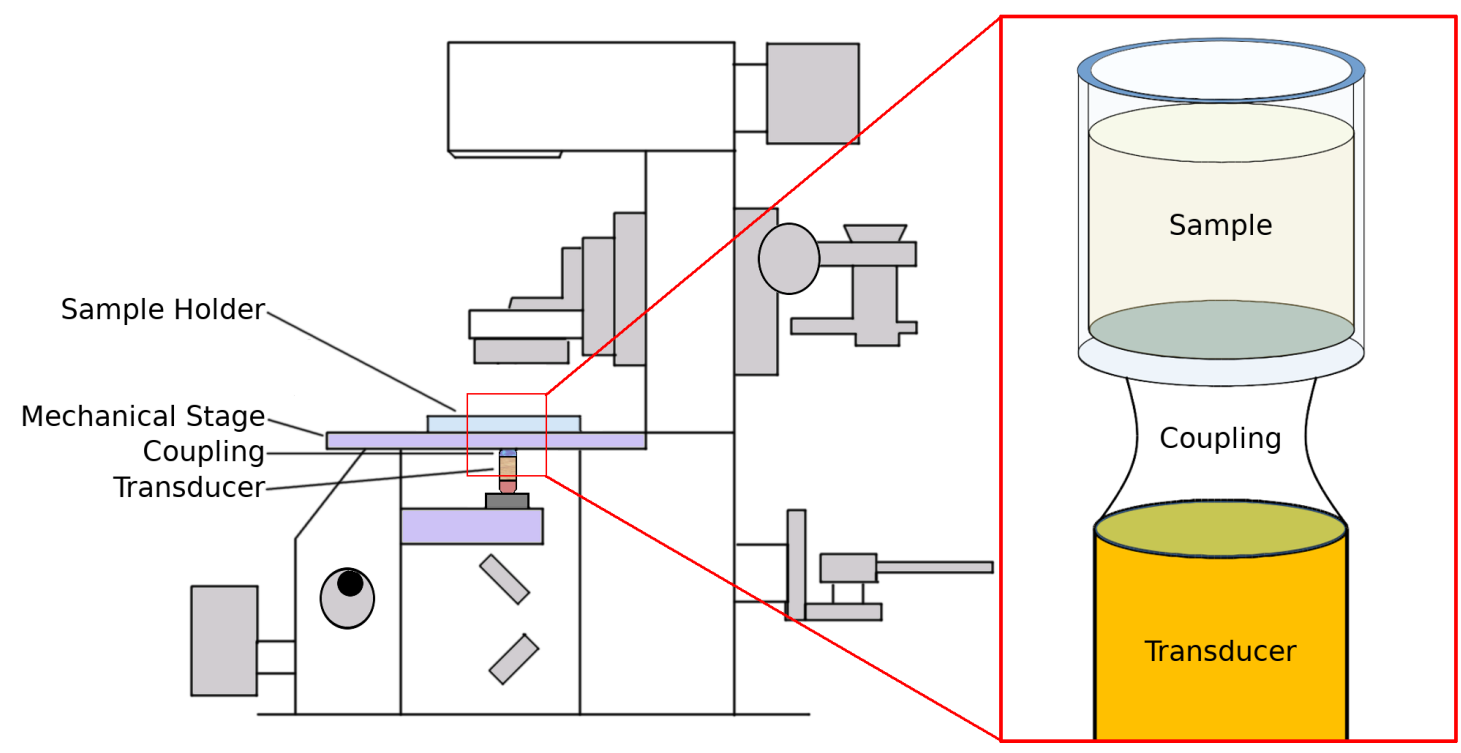

Figure 3.2: The SASAM with the system modifications made for this work is shown. The mechanical stage and adjacent wells of the microtitre plate have been removed in the enlarged schematic for better visualization. SASAM schematic (left) is based of off work by Rui et al. [76].

collected. A pulse repetition frequency of $24.24 \mathrm{kHz}$ was used and each saved RF line was the average of 50 recorded pulses. Averaging increased the SNR by smoothing out any random noise present in the signal.

Added spatial information was obtained by collecting fifty RF lines in $140 \mu \mathrm{m}$ steps. The transducer remained fixed, while the sample was shifted using the mechanical stage. The scan line covered a $7 \mathrm{~mm}$ span and therefore encompassed a complete cross-section of the $\sim 6.4 \mathrm{~mm}$ well. This allowed for the creation of a crude image.

\subsubsection{Transducer specifications}

A single-element focused transducer with a central frequency of $80 \mathrm{MHz}$ was used. It was constructed from lithium niobate $\left(\mathrm{LiNbO}_{3}\right)$ at the University of Southern California's Ultrasonic Transducer Resource Center (UTRC). The aperture diameter is listed as $2 \mathrm{~mm}$ with an F\# of 3. All transducer specifications can be found in Tab. 3.1, where the values were calculated using equations discussed in Sec. 2.1.3. For the calculations, a sound speed of $1.5 \times 10^{3} \mathrm{~m} / \mathrm{s}$ was assumed and the pulse length was approximated using the transducer bandwidth depicted in Fig. 3.4. An image of the transducer is shown in Fig. 3.3. Distortion caused by the electronics resulted in alterations from the intended mono-cycle pulse shape that is typically seen in single-element focused transducers. The distortions also caused the bi-modal shape in the normalized frequency spectrum. As a result, the axial resolution was 


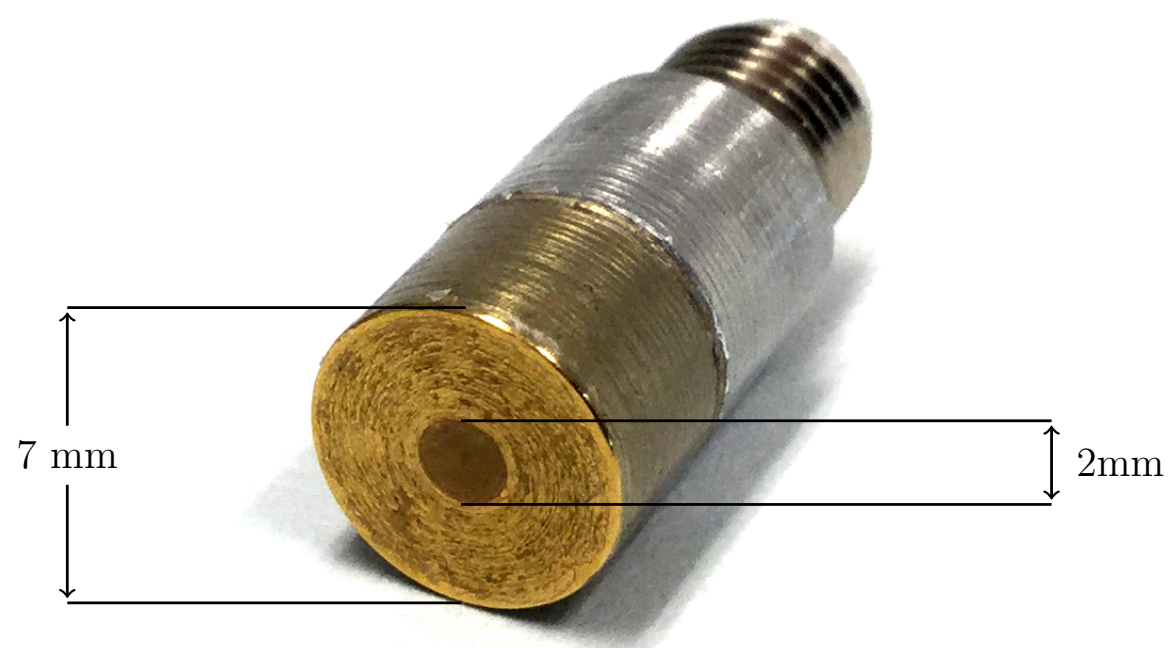

Figure 3.3: The $80 \mathrm{MHz}$ transducer is shown, with a $7 \mathrm{~mm}$ total diameter and a $2 \mathrm{~mm}$ aperture diameter.

larger than the value listed in Tab. 3.1.

\begin{tabular}{lr}
\hline \hline Central Frequency & $80 \mathrm{MHz}$ \\
Bandwidth & $\sim 60 \mathrm{MHz}$ \\
Aperture Radius & $1 \mathrm{~mm}$ \\
Focal Length & $6 \mathrm{~mm}$ \\
Lateral Resolution & $58.9 \mu \mathrm{m}$ \\
Axial Resolution & $12.5 \mu \mathrm{m}$ \\
Aperture Angle & $9.5^{\circ}$ \\
Numerical Aperture & 0.16 \\
F \# & 3 \\
\hline \hline
\end{tabular}

Table 3.1: Transducer Specifications.

\subsubsection{Typical acquisitions}

A schematic of the the modified set-up used for this work is shown in Fig. 3.5 alongside a typical RF line. The schematic shows echos from three interfaces, which for illustrative purposes are depicted using curved arrows. In reality, the transmitted pulse and the echos lie along a single path, aligned with the centre of the transducer. RF lines like the one shown in Fig. 3.5 were acquired every 30 seconds at 50 lateral scan locations by shifting the alignment of the microtitre plate using the mechanical stage. 

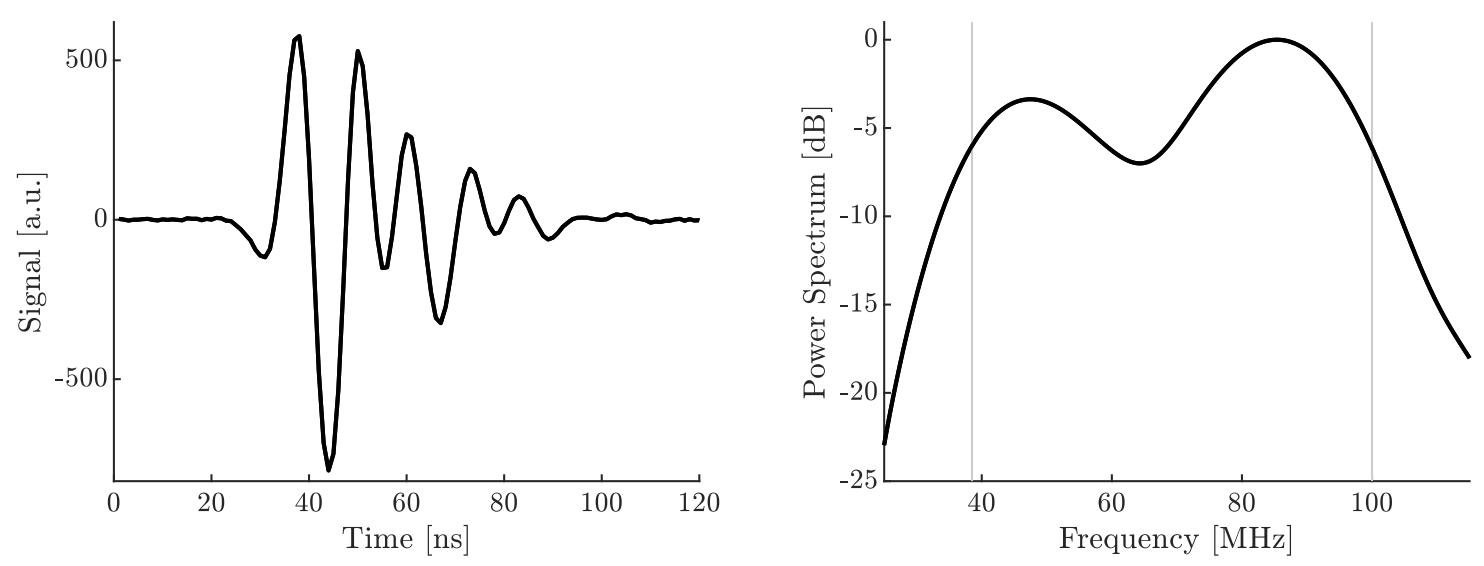

Figure 3.4: The signal and power spectrum are shown from an echo off of a smooth polystyrene surface. The grey lines on the power spectrum estimate the $6 \mathrm{~dB}$ bandwidth.

Prior to the start of each experiment the distance between the transducer and the well was adjusted to ensure that the water droplet effectively coupled the two surfaces. In order to satisfy this requirement and achieve consistency between experiments, the height of the transducer was set so that the reflection from the second interface had an echo time $\left(t_{2}\right)$ of approximately $6000 \mathrm{~ns}$. This corresponded to $\sim 3.9 \mathrm{~mm}$ between the transducer's surface and the bottom surface of the well. As discussed in Sec. 2.1.1, large echos at boundaries between different materials occur as a result of a mismatch in the acoustic impedance. The echo time depended on both the distance that the wave travelled and the acoustic velocity in that medium. Here, two reflections occurred due to the layer of plastic that separated the sample from the coupling medium, while the third occurred at the top of the sample. When whole blood was used, backscattered signal caused by the presence of red blood cells was also visible in the region between $t_{2}$ and $t_{3}$. For these experiments, parameters were examined pertaining to (a) the time of flight through the sample $\left(t_{s}\right)$ (b) the motion of scatterers within the sample (between $t_{2}$ and $t_{3}$ ) and (c) frequency dependant changes in the third echo $\left(t_{3}\right)$ which will provide information about attenuation of the pulse as it passes through the sample. The remainder of this section will focus on the extraction of each of these parameters from RF line data.

\section{Time of flight}

In early experiments, the third echo corresponded to an interface between the sample and the air. In these cases, the time of flight through the sample $\left(t_{s}\right.$ in Fig. 3.5) could change as a result of both acoustic velocity changes or due to physical changes in the location of the sample's surface. Since it was not possible to isolate these two variables, data was expressed using the change in the time of flight through the sample relative to the first measurement. 

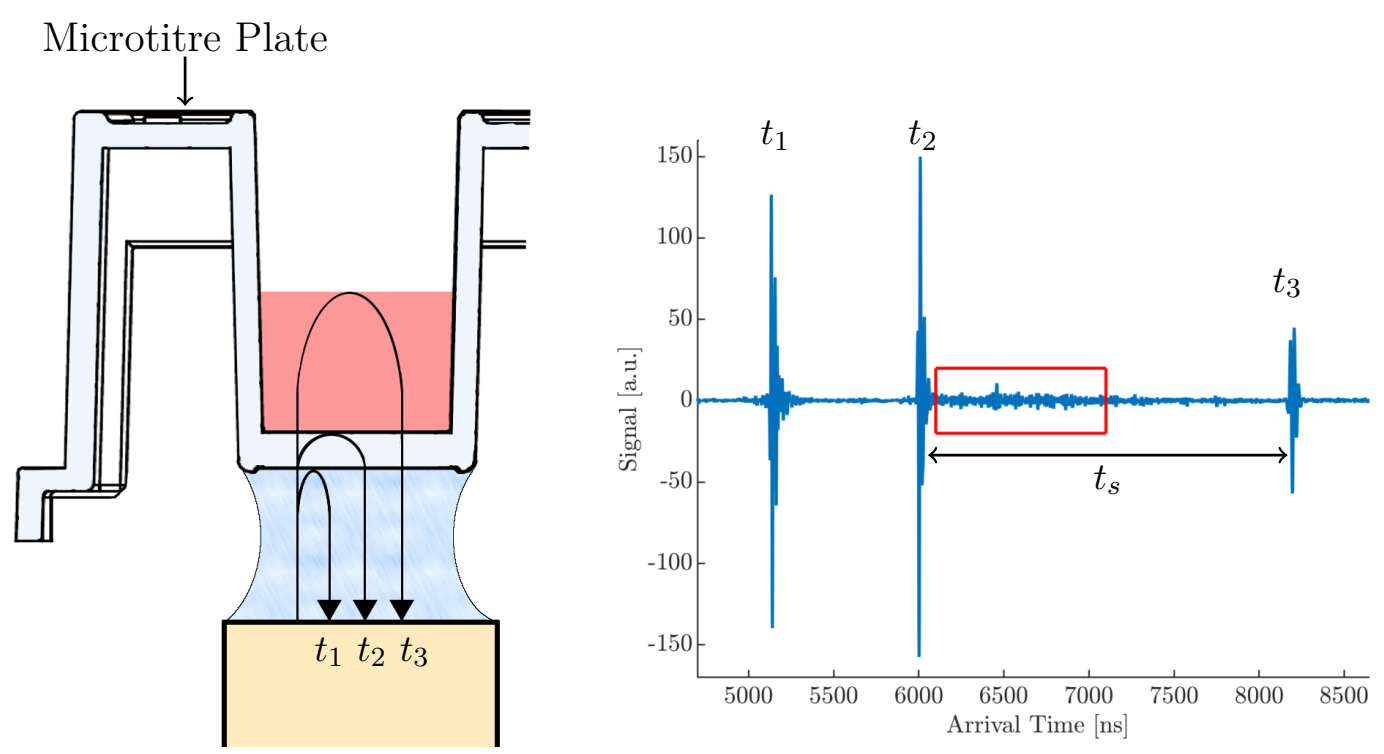

Transducer

Figure 3.5: A schematic of the sample contained in a single well of a 96-well microtitre plate which was coupled to the transducer using a droplet of water. Acoustic reflections at the boundaries are indicated by $t_{1}, t_{2}$, and $t_{3}$ and the corresponding RF line is shown. The time of flight through the sample $\left(t_{s}\right)$ is indicated, and a region of interest showing RBC backscatter is boxed in red.

The change observed at the $i^{\text {th }}$ time point was determined by:

$$
\Delta t_{i}=t_{s, i}-t_{s, 0}
$$

where $t_{s, i}$ is the time flight through the sample at the $i^{\text {th }}$ time point and $t_{s, 0}$ is the time of flight through the sample measured in the first acquisition.

In later experiments and for the majority of the data presented in this work, the distance travelled through the sample was fixed by introducing an acrylic cap that was immersed into the sample (see Fig. 3.6). Consequently, the third echo corresponded to the reflection from the acrylic cap rather than air. Although the distance travelled by the acoustic wave between the bottom of the well and the plug remained constant within a given experiment, slight variability in the cap placement meant that it was not possible to accurately measure the distance that the wave travelled. As such, it was decided to express the time of flight information in terms of percentage change of the acoustic velocity, which is independent of the distance.

For a fixed distance $d$ between the bottom of the well and the cap travelled by a wave with an acoustic velocity $c$ in the sample, the time of flight is: 


$$
t_{s}=\frac{2 d}{c}
$$

Conversely, the acoustic velocity can be expressed in terms of the distance travelled and the time of flight as:

$$
c=\frac{2 d}{t_{s}}
$$

To determine the percentage change in acoustic velocity at time $i$ relative to the acoustic velocity at the initial time point, the relationship described in Eq. 3.5 can be used. These values were calculated at each 30 second time point for the duration of the experiment.

$$
\begin{aligned}
\Delta c_{i}[\%] & =\left(\frac{c_{i}-c_{0}}{c_{0}}\right) \times 100 \%=\left(\frac{\left(2 d / t_{s, i}\right)-\left(2 d / t_{s, 0}\right)}{\left(2 d / t_{s, 0}\right)}\right) \times 100 \% \\
& =\left(\frac{t_{s, 0}}{t_{s, i}}-1\right) \times 100 \%
\end{aligned}
$$

\section{Scatterer motion}

The red box overlayed onto the RF line in Fig. 3.5 indicates a region near the bottom of the well where the backscatter signal from the RBC was largest. This was in part due to their proximity to the transducer (less of the wave had been attenuated) and also due to their proximity to the transducer's focus, which lay near 6400 ns after having passed through the plastic. This corresponds to a focus at $\sim 5.2 \mathrm{~mm}$ from the transducer after passing through the $1 \mathrm{~mm}$ layer of plastic at the bottom of the well, compared to a focus at $\sim 5.8 \mathrm{~mm}$ when the wave passes through water only.

As the scatterers moved within the sample, the backscatter signal fluctuated. The variations in the scatterer motions were monitored using M-Mode images (Sec. 2.1.4). The region indicated by the red box is the region that was used to construct the M-Mode images.

\section{Reflection Spectra}

The frequency spectrum of the third echo $\left(t_{3}\right)$ was obtained by centring the echo signal in a 400 ns window, and using MATLAB's fft function to perform a Fourier transform on the gated data. This was done for each time point of the experiment in order to observe how the frequency domain data changed. 
As the Fourier transforms could contain imaginary components, the raw frequency data $(F)$ was converted to the normalized power spectrum $(S)$ in decibels using the following equation:

$$
S=20 \log _{10}\left(\frac{|F|}{\left|F_{\text {ref }}\right|}\right),
$$

where the normalization factor $F_{\text {ref }}$ was frequency dependent, meaning that each frequency component of the spectrum underwent different normalizations. The normalization factor was chosen to be the maximum value that was recorded for a particular frequency when considering all of the acquired time points. This normalization made it easier to observe the overall changes that occurred at each frequency. These normalized frequency spectra shall be referred to as the reflection spectra in this work.

\subsection{Experimental refinement}

In the development of this new measurement system, a number of issues were identified while attempting to replicate the protocol used in the OHP setup. These are discussed in the following sections along with how each issue was addressed.

\subsubsection{Meniscus}

Early iterations of the plasma experiments were performed with RF-lines acquired at a single location rather than with the 50 point scan-line currently in use. It was observed that the reflection off the surface of the clot had highly variable amplitudes and in certain situations would not be visible in the RF line at all. A 2D scan of the well containing $100 \mu \mathrm{L}$ of water revealed that the signal intensity at the clot-air interface was strongly dependant on location. The variation in signal intensity was attributed to the curvature of the meniscus. The spatial dependence of the signal intensity acquired in this scan be seen in Appendix. B.1.

Acquiring a 50 point cross-section of the well generally ensured that a subset of the data contained reflections from the clot-air interface. Later experiments incorporated a well cap, which, as discussed in Sec. 3.2.5, ensured that a reliable signal was acquired at the centre of the well. 


\subsubsection{Evaporation}

Plate readers used in OHP tests rely on optical transmittance through the sample. As such, lids or alternative well coverings are typically avoided as they can directly interfere with the optical data. However, in the context of US experiments evaporation can reduce the sample volume and directly impact time of flight measurements. In order to assess the extent of the evaporation as well as the effectiveness of certain potential solutions, measurements were acquired for $37^{\circ} \mathrm{C}$ water for three grades of well coverage: (i) uncovered, (ii) topped with the loose-fitting 96-well plate lids, and (iii) sealed with a strip of adhesive. Volume loss in the uncovered well was estimated to be $30 \mu \mathrm{L} / \mathrm{hr}$. This was reduced to $12 \mu \mathrm{L} / \mathrm{hr}$ using the loose fitting lid and to $2 \mu \mathrm{L} / \mathrm{hr}$ by using the adhesive strip. It was concluded that the well should be sealed to minimize evaporation effects. Later experiments rely on the cap described in Sec. 3.2.5 to minimize evaporation rather than the adhesive strip that was originally used.

\subsubsection{Temperature}

As with evaporation, temperature was of minimal concern in the original protocol designed for the microtitre plate reader as it has a negligible impact on optical results. However, the acoustic properties of a medium vary with temperature. Thus, the ideal situation for US measurements is that all sample constituents are warmed to $37^{\circ} \mathrm{C}$ prior to data acquisition. A complication to this is that pre-heating the enzymes could impact their activity.

Experiments were performed to determine the change in time of flight for samples prepared with three temperature profiles. These were performed in water with the well sealed using an adhesive strip. Assuming negligible evaporation, all time of flight changes should correspond directly to temperature-induced sound speed variations. It was determined that pre-warming $88 \%$ of the sample volume to $37^{\circ} \mathrm{C}$ while keeping the remaining $12 \%$ on ice yielded similar changes to those observed when $100 \%$ of the sample was pre-warmed to $37^{\circ} \mathrm{C}$. In the context of coagulation experiments, this meant that keeping the enzymes on ice while the remainder of the sample was warmed did not result in significant temperature dependent changes in the acoustic properties.

\subsubsection{Acquisition automation}

The plate reader system automatically acquires optical density readings every minute and continues to do so until either the acquisition is stopped, or the predefined number if data points has been reached. The Acoustic Investigator software does not have the capability to acquire scan-lines periodically. As a result, if periodic data is to be acquired, it must be 


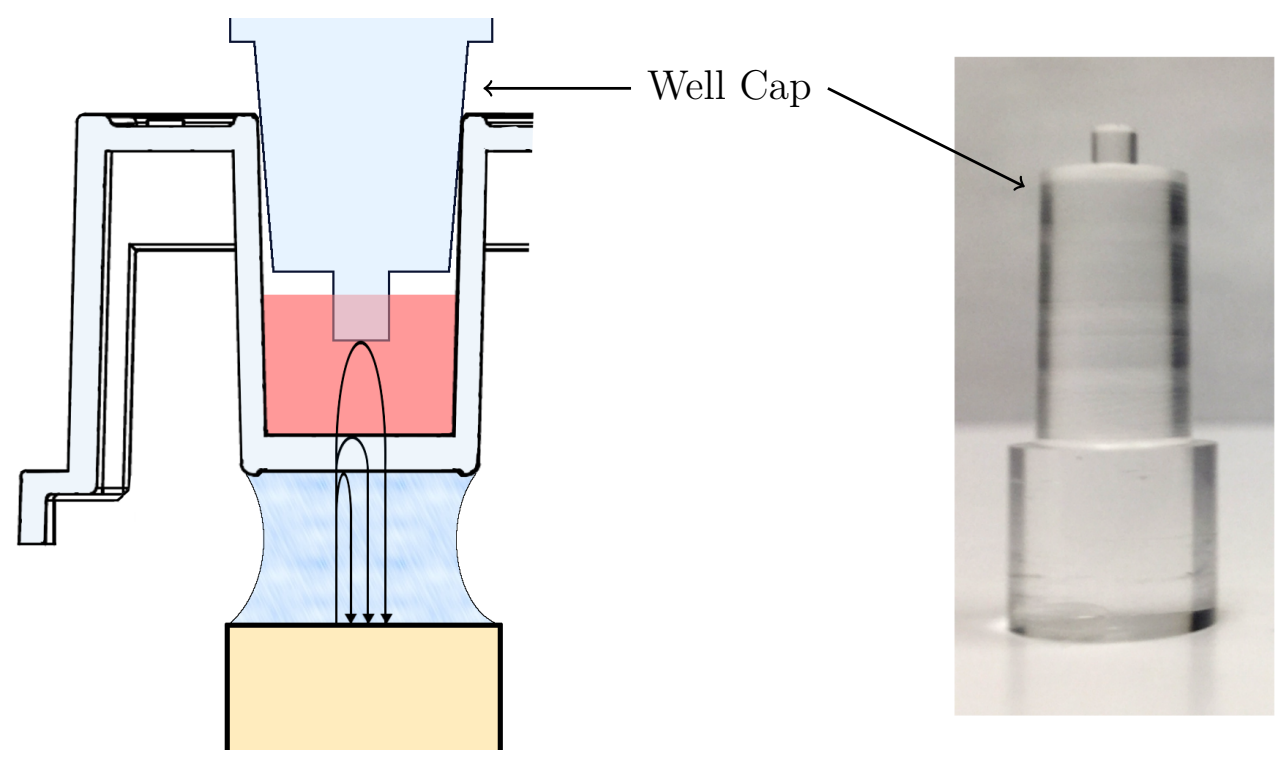

Figure 3.6: The well cap designed to seal the well and fix distance travelled by the acoustic wave in a schematic (left) and an image (right)

done manually. Until an alternative solution was found, this entailed manually starting and saving each scan-line. To monitor coagulation and fibrinolysis in a single sample, this could translate to saving data every 30-60 seconds continuously for up to an hour. Typically, 10-15 samples were performed per day. Rather than attempting to modify the Acoustic Investigator software, a short script was written to mimic the series of mouse clicks and keyboard entries required to save a scan-line. It was then set to repeat periodically and continue doing so until the desired number of acquisitions were complete or until the program was manually interrupted. The scripting language used is called AutoIt (AutoIt Consulting Ltd., Wales) and the script written for the automation is provided in Appendix B.2.

\subsubsection{Well cap}

The well cap was designed in an attempt to isolate changes in the sound speed from those in the distance travelled by the acoustic wave. As has been discussed in Sections 3.2.1 and 3.2.2, the meniscus and evaporation can both impact the surface height of the liquid column. Results obtained in plasma show that changes in the sample can also impact the surface height (Sec. 4.1).

An image and schematic of the well cap can be seen in Fig. 3.6.

It was designed to fit snugly into the well so that approximately $1 \mathrm{~mm}$ of the protruding column was immersed in the sample material. The $2 \mathrm{~mm}$ diameter of the column allowed for up to $15 \mathrm{RF}$-lines to be acquired that include a reflection from the surface. By fixing the 
distance that the US wave travelled, time of flight depended exclusively on changes in the sound speed of the medium.

\subsection{Coagulation and Fybrinolytic Assay}

Two main approaches were taken in developing designing the experiments. In the first, acellular samples (lacking cells) composed of human blood plasma were examined. For these sets of experiments, the kinetics of clot formation and breakdown could be well characterized using optical techniques, as plasma is optically transparent. This aided in calibrating the enzyme concentrations to achieve clot formation and breakdown within the desired time. Three sets of experiments were performed: (i) a control set where no coagulation was initiated, (ii) a set where only coagulation was initiated (using thrombin as an activating agent), and (iii) a set where both coagulation and fibrinolysis were initiated (using thrombin as an activating agent, and $\mathrm{u}-\mathrm{PA}$ as a lytic agent).

In the cellular clots, which were performed using murine whole blood, optical calibration of enzyme titrations was not possible as whole blood is not optically transparent. Additionally, resistance to fibrinolysis in murine blood resulted in the inability to test the fibrinolytic pathway. As such, two types of whole blood samples were examined: (i) a control set where no coagulation was initiated, and (ii) a set where only coagulation was initiated using calcium chloride as an activating agent. The calcium chloride results in a much slower onset than the thrombin, which allows for better visualization of the clot formation. Additionally, calcium chloride was the activating agent in the literature where US was used to monitor blood coagulation [44].

\subsubsection{Buffer preparations}

Three buffer solutions are required for the coagulation and fibrinolytic experiments. In order to minimize the risk of bacterial contamination, new buffers were prepared monthly or as needed. The specific protocols including exact volumes that were used for each buffer are provided in Appendix A. A general description is given below.

\section{Blocking Solution}

The blocking solution was a 1\% solution of Tween@20 (P2287, Sigma-Aldrich) in HEPESbuffered Saline (HBS; BB-553, Boston BioProducts). The HBS arrived at 5X concentration, and was diluted with MilliQ filtered water (Millipore Corporation, USA) to achieve $25 \mathrm{mM}$ HEPES and $150 \mathrm{mM}$ Sodium Chloride (pH 7.4). The solution was used to coat the microtitre 
plates and the cap described in Sec. 3.2.5. The plate was coated by filling the wells and the cap was fully immersed into the solution. The plate and cap were set aside for 1-2 hours before they were rinsed in MilliQ water and allowed to dry. Since the caps needed to be reused, they were washed and re-immersed in the solution for 20 minutes between experiments. The blocking solution served to wash the plate of any potential contamination (dust, etc.), while also providing a thin coating on the surface of the plastic that minimized the risk of blood enzyme activation.

\section{Assay buffer}

The assay buffer was used for all stock dilutions that did not involve enzymes. It was a solution of $0.01 \%$ Tween $囚 20$ in HBS (25 mM HEPES, 150 mM Sodium Chloride, pH 7.4).

\section{Prionex buffer}

The Prionex buffer was used for all enzyme dilutions, as Prionex has excellent protein stabilizing properties. This buffer was made by adding 0.1\% Prionex (24621-100, Polysciences Inc., USA) to the assay buffer.

\subsubsection{Stock enzyme dilutions}

\section{Human alpha thrombin}

Human alpha thrombin (HT 1002a, Enzyme Research Laboratories) was used to initiate the transformation of fibrinogen into fibrin, ultimately resulting in clot formation. One sample contained $1000 \mathrm{NIH}$ units (a standardized measure of enzymatic activity) and arrived as a frozen liquid, typically with a volume less than $100 \mu \mathrm{L}$. Slight variations in the volume occurred as a result of inter-batch variability in the enzymatic activity.

The product was stored at $-80^{\circ} \mathrm{C}$ until it was ready to be diluted. For the initial dilution, the stock was diluted to a concentration of $1000 \mathrm{NIH}$ units/mL by adding enough prionex buffer to bring the total volume of the solution to $1 \mathrm{~mL}$. For example, if the stock volume was $94 \mu \mathrm{L}, 846 \mu \mathrm{L}$ of buffer were added. The solution was filtered through a $200 \mathrm{~nm}$ PES filter before being divided into $15 \mu \mathrm{L}$ aliquots. These aliquots were refrozen at $-80^{\circ} \mathrm{C}$ and stored for later use. Further dilutions were performed on the day of the experiments.

\section{Urokinase plasminogen activator}

Urokinase plasminogen activator (u-PA; ENZ-264, ProSpec) was used to initiate the breakdown of fibrin polymers through the activation of plasminogen into plasmin, thereby causing 
clot breakdown. The product was ordered in $1 \mathrm{mg}$ units and arrived as a lyophilized (freezedried) powder. Prior to dilution, the powder was stored at $-20^{\circ} \mathrm{C}$. The $\mathrm{u}-\mathrm{PA}$ solution was produced by adding $1 \mathrm{~mL}$ of distilled water. This resulted in a solution of $1 \mathrm{mg} / \mathrm{mol}$, corresponding to a stock concentration of $18.5 \mu \mathrm{M}$. As was done with the thrombin, the solution was divided into $15 \mu \mathrm{L}$ aliquots and stored at $-80^{\circ} \mathrm{C}$. It was further diluted at the time of the experiments.

\subsubsection{Acellular clot composition}

A standard guideline was developed for the composition of acellular clots that was loosely based off of the OHP protocols. Acellular clots refer to clots generated using pooled platelet poor human blood plasma (CCN-10, CryoCheck, PrecisionBioLogic), where blood cells have been removed and fewer than 10,000 platelets per microlitre are present. In order to draw comparisons to the OHP measurements, the clot was designed in such a way as to meet the requirements of both systems. Since the plate reader measures optical density and thus relies on optical transmittance, accurate readings are difficult when the sample is opaque. A total sample volume of $100 \mu \mathrm{L}$ was used. This $100 \mu \mathrm{L}$ sample contained $5 \mu \mathrm{L}$ of IIa, $5 \mu \mathrm{L}$ of u-PA, and $2 \mu \mathrm{L}$ of calcium chloride (Calciject, Omega Laboratories Ltd., Montreal, Canada). The remaining volume was dilute human blood plasma, with $33 \mu \mathrm{L}$ of plasma in $55 \mu \mathrm{L}$ buffer. The dilution ensured that the sample was not too opaque for optical measurements.

Various titrations of u-PA were tested in order to achieve a desired lysis time. A working concentration of $20 \mathrm{nM}$ was found to cause lysis within 20 minutes of clot initiation and was chosen in order to guarantee that decoupling of the US transducer did not occur before the clot had fully broken down. Thrombin $(5 \mathrm{~nm})$ and calcium chloride $(15 \mathrm{mM})$ concentrations were chosen based on common literature values. For the control experiments, wherein one or more enzymes were not added to the sample, the thrombin and calcium chloride volumes were replaced by prionex buffer.

\subsubsection{Whole blood clot composition}

Where plasma is a mixture of water and proteins that can be frozen and still preserve its integrity, whole blood contains cells that are altered with age and storage, which in turn can impact the coagulation kinetics. Given the storage time associated with blood obtained from the Canadian Blood Services and the difficulty in obtaining ethics board approval for fresh blood samples greater than $50 \mu \mathrm{L}$, mouse blood was used as an alternative.

When moving to whole blood, comparisons to spectrophotometric tests were no longer feasible. As a balance between the OHP and US system was no longer necessary, the protocol 
used for plasma clots was modified to create samples that provide a more realistic clot environment. This involved maximizing the amount of citrated whole blood in the sample by eliminating the buffer dilution.

Translatability between mouse blood and human blood was previously discussed in Sec. 2.2.2. The main concern is that coagulated murine blood is highly resistant to clot breakdown relative to human blood. Following unsuccessful attempts to break down clotted mouse blood, u-PA was eliminated from these experiments. A benefit of using mouse blood over human blood, however, is that the onset of clot formation using exclusively calcium chloride is significantly ( $\sim 4$ times) shorter than the onset seen in human blood [16]. Thus, thrombin was not needed to initiate clot formation within the desired time-frame. These changes (elimination of buffer and thrombin) ultimately resulted in a final clot composition of $98 \mu \mathrm{L}$ citrated mouse blood with $2 \mu \mathrm{L}$ of calcium chloride, such that the final concentration of calcium chloride in the well was $15 \mathrm{mM}$.

\subsubsection{Sample preparation}

The well caps and plate were blocked for 1-2 hours as described in Sec. 3.3.1. Once they had been blocked and washed, they were placed inside the $37^{\circ} \mathrm{C}$ chamber that enclosed the SASAM for pre-warming.

\section{Plasma Sample Preparation}

Three types of samples were prepared using the plasma. The first was a control sample, where the thrombin, u-PA, and calcium chloride were replaced by $12 \mu \mathrm{L}$ of assay buffer. In this case, coagulation was not initiated and therefore the sample should remain in a liquid state throughout the experiment. In the second, only thrombin and calcium were added, while the u-PA was replaced by $5 \mu \mathrm{L}$ of assay buffer. The thrombin and calcium chloride initiated clot formation and, in the absence of any fibrinolytic agents, the clot should stay formed for the duration of the experiment. The final sample was meant to emulate the OHP test, with all three of the aforementioned constituents added to the sample. The enzyme concentrations were chosen such that the clot onset happened within minutes of the mixing and clot breakdown occurred roughly 20 minutes into the experiment.

Prior to the experiments, the frozen thrombin and u-PA were thawed, diluted to the concentrations mentioned in Sec. 3.3.3, and put back on ice immediately. The pooled plasma was thawed according to the manufacturer's instructions. This involved placing the sample in a $37^{\circ} \mathrm{C}$ water bath for 4 minutes (in the case of $1 \mathrm{~mL}$ plasma volumes) and placing it on ice. 
Once all of the necessary materials had been gathered, a $120 \mu \mathrm{L}$ volume of 3:5 plasma to buffer mixture was placed in a $500 \mu \mathrm{L}$ microcentrifuge tube and put in the chamber to warm for 5 minutes. Once 4 minutes had passed, the pre-warmed microtitre plate was removed from the chamber. Thrombin $(5 \mu \mathrm{L})$, u-PA $(5 \mu \mathrm{L})$ and calcium chloride $(2 \mu \mathrm{L})$ (or their buffer substitutions) were added to the bottom of the well such that each droplet occupied its own third and did not mix with the other constituents. After the plasma mixture had been in the chamber for 5 minutes, it was removed and $88 \mu \mathrm{L}$ of the solution was pipetted into the well containing the enzymes. The solution was mixed by pipetting a portion of the volume up and down 4-5 times and mixing the sample in a circular motion using the pipette tip as a stir stick. The well was then sealed using either an adhesive strip or the well cap. A water droplet was placed on top of the transducer and the 96 well plate was placed onto the stage and coupled to the drop of water. Transducer alignment was verified by ensuring the visibility of a 3rd echo in the displayed RF-line data. If the alignment was correct, the acquisition was started either manually or using the automation script. The delay between the addition of the plasma to the enzymes and the first set of data acquisition was typically 30-60 seconds.

\section{Whole Blood Preparation}

For the whole blood experiments, two types of samples were monitored. The first was a control, where the aforementioned $2 \mu \mathrm{L}$ of calcium chloride (Sec. 3.3.4) was replaced by $2 \mu \mathrm{L}$ of assay buffer. This sample should not undergo coagulation. The second sample involved the $98 \mu \mathrm{L}$ of whole blood, where clot formation was initiated using $2 \mu \mathrm{L}$ of calcium chloride. The samples containing the calcium chloride are often referred to as recalcified samples to indicate that calcium has been introduced. Recalcified samples should undergo coagulation.

Prior to the experiments, the calcium chloride was diluted to the desired working concentration of $15 \mathrm{mM}$. The blood samples were drawn on the morning of the experiments into $3.2 \%$ citrate tubes to prevent undesired clot formation. From the citrate tube, $130 \mu \mathrm{L}$ sample of the blood was extracted into a $500 \mu \mathrm{L}$ microcentrifuge tube and placed in the $37^{\circ} \mathrm{C}$ chamber to warm for 10 minutes. When 1 minute remained, the microtitre plate was removed from the chamber and $2 \mu \mathrm{L}$ of calcium chloride was added to the centre of a well. The blood was removed from the chamber and $98 \mu \mathrm{L}$ was pipetted onto the calcium chloride. The solution was mixed up and down 4-5 times and stirred in a circular motion. Once the mixing was complete the well was sealed using a well cap. The plate was coupled to the transducer using a droplet of water and the acquisition was started via the automated script.

With the whole blood, lack of clot formation (controls) and clot formation (for all other measurements) were verified at the end of each experiment by inverting the 96 well plate 
a)

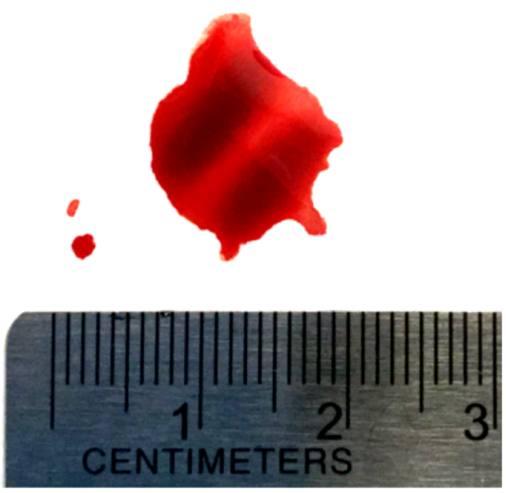

b)
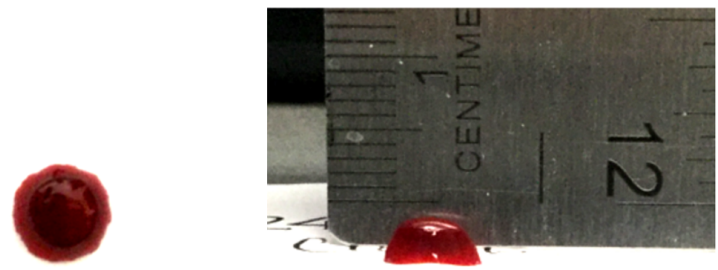

Figure 3.7: Two samples are shown at the end of an experiment. a) a control sample, where the calcium was replaced by buffer, and b) a coagulated sample viewed from the top and from the side.

onto a sheet of paper. This verified that undesired clot formation did not occur in the case of controls, which can occasionally occur due to contact activation of the intrinsic pathway (see Sec. 2.2.1). It also verifies that the samples that were activated using calcium chloride resulted in clot formation. An image of a liquid and a coagulated sample is shown in Fig. 3.7. It can be seen that the coagulated sample retained the shape of the well, which had a diameter of $\sim 6.4 \mathrm{~mm}$, while the liquid sample was dispersed over a larger area.

\subsubsection{Summary of Experiments}

\section{Plasma Samples}

Experiments in plasma were performed with and without the well cap for the three sample compositions discussed in Sec. 3.3.5. Prior to the well cap's implementation, three control replicates were acquired along with four samples that coagulated and four samples that underwent both coagulation and fibrinolysis. With the well cap, each sample type was replicated five times. Information regarding the replicates for all sample types can be found in Tab. 3.2. The results from the plasma experiments are shown in Sec. 4.1, and discussed in Sec. 5.1 .

\section{Murine Whole Blood}

Four sets of experiments across three mouse strains were performed using murine whole blood. The strains used were CD1, CCM2 Flox, and C57 which were chosen based on availability on the day of the experiment. No literature reported any hemostatic irregularities in these strains. The number of samples performed on a given day was dependant on the 
Plasma Sample Replicates

\begin{tabular}{lccc}
\hline \hline Sample Details & Control & Coagulation & $\begin{array}{c}\text { Coagulation } \\
\text { \& Fibrinolysis }\end{array}$ \\
\hline No cap & 3 & 4 & 4 \\
With cap & 5 & 5 & 5 \\
\hline \hline
\end{tabular}

Murine Whole Blood Sample Replicates

\begin{tabular}{lccc}
\hline \hline Sample Details & Control & Coagulation & $\begin{array}{c}\text { Coagulation } \\
\text { \& Fibrinolysis }\end{array}$ \\
\hline CD1 (set 1) & 3 & 3 & - \\
CD1 (set 2) & 3 & 3 & - \\
CCM2 Flox & 4 & 4 & - \\
C57 & 3 & 6 & - \\
\hline \hline
\end{tabular}

Table 3.2: The number of replicates for each type of plasma or murine whole blood experiment. Plasma replicates are broken into experiments performed with and without well caps. Whole blood replicates are broken into experiments by mouse strain, where the CD1 strain is split over two sets to indicate that each set was acquired on a different day.

amount of blood obtained, which varied depending on the size of the mice from which the sample was taken.

Experiments using the CD1 blood were performed on two separate days, using fresh blood samples each time. Three recalcified samples were measured on each day, which amounted to a total of $6 \mathrm{CD} 1$ samples that underwent coagulation. A total of six control experiments were also performed in samples where the calcium was substituted with buffer. The CCM2 Flox experiments were done on a single day, on which measurements were made of four recalcified and four control samples. The C57 experiments were also performed on a single day. Six recalcified samples were acquired along with three controls. 


\section{Chapter 4}

\section{Results}

\subsection{Human Blood Plasma}

For the plasma experiments, a large portion of the data collection was performed prior to the major system improvements made. The most impactful change was the introduction of the well cap. Data collected with and without the use of the well cap are presented here and discussed in the following chapter.

Platelet poor plasma is relatively homogeneous and the size of scatterer required to produce a sufficient backscatter signal from an $80 \mathrm{MHz}$ acoustic wave is much larger than what is needed for optical studies. The main information was thus collected by examining reflections of the wave at interfaces between two media. Examining the sample interface furthest from the transducer $\left(t_{3}\right.$ in Fig. 3.5)provided the best measurement of acoustic changes that occurred within the medium.

As discussed in Sec. 3.1.5, each experiment involved acquiring data along a cross-section of the well by collecting 50 RF-lines in $140 \mu \mathrm{m}$ steps. This was performed periodically throughout the duration of the experiment. Two representative B-Mode images, constructed using the 50 RF-lines, are shown in Fig. 4.1 for plasma samples with and without the wellcap. 
a)

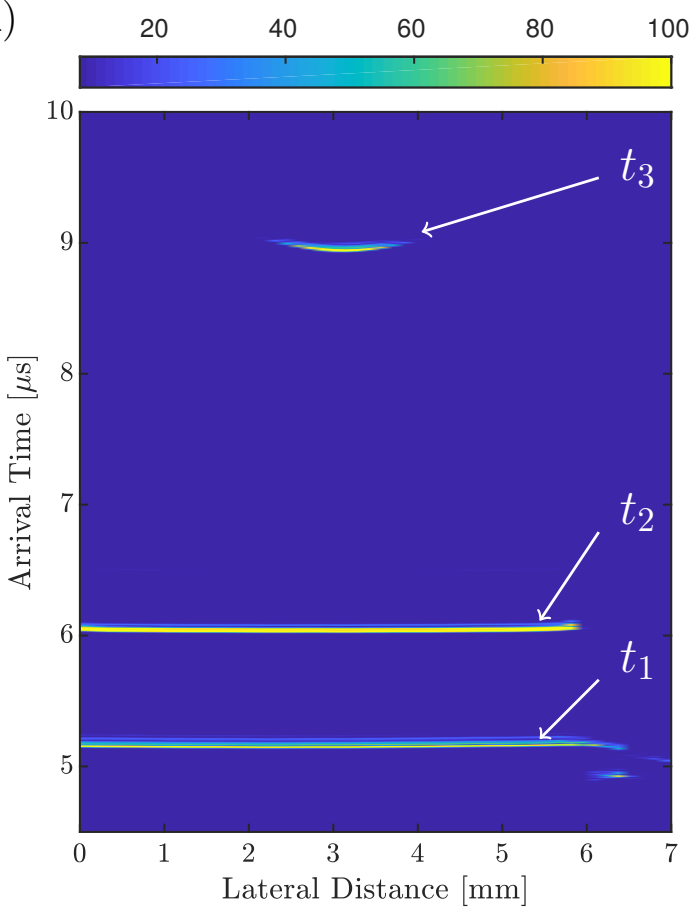

b)

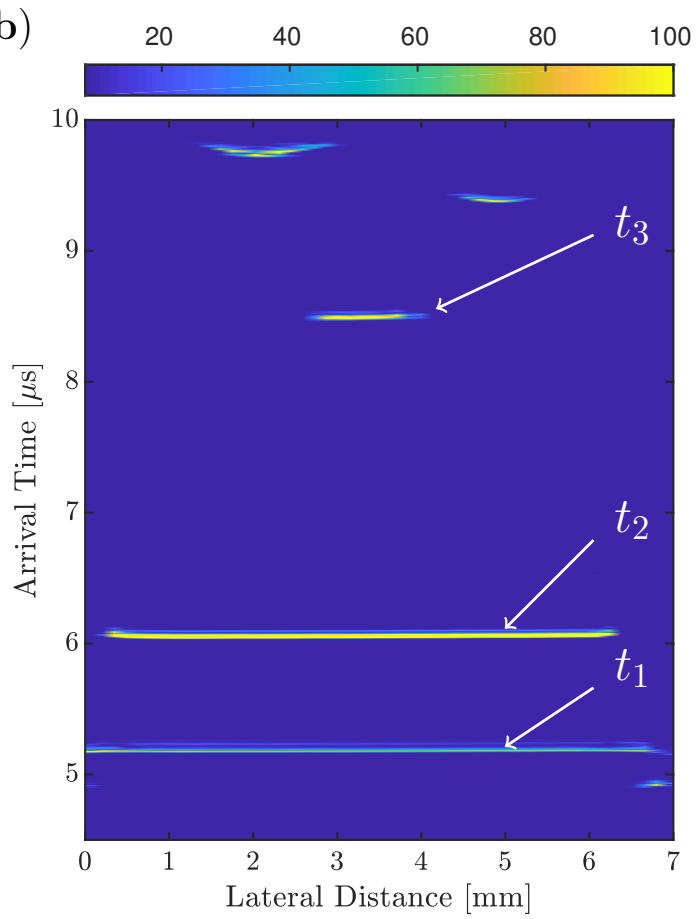

Figure 4.1: B-Mode images for two plasma samples: (a) without the plug, and (b) with the plug in place. The bottom two interfaces $\left(t_{1}\right.$ and $\left.t_{2}\right)$ correspond to the layer of plastic separating the transducer from the sample. In a) the meniscus of the sample-air interface is seen at the top of the sample $\left(t_{3}\right)$, while in b) the interface at the centre corresponds to the surface of the cap $\left(t_{3}\right)$, and the two menisci from the sample-air interface can be seen at the top of the frame on either side of the cap. Colour scale is linear and was truncated at 100 a.u. for proper visualization of all interfaces.

\subsubsection{Prior to Well-Cap}

The time of flight data, which was calculated as described in Sec. 3.1.5, is shown in Fig. 4.2 for the three sample types outlined in Sec. 3.3.5. Each line represents the mean of either three or four datasets (indicated in the legend), while the standard deviation is shown as a shaded region in the same colour.

\subsubsection{Using the Well-Cap}

With the introduction of the cap, the US wave reflection from the plasma-cap interface was used. Since the distance the US wave travelled remained constant throughout the experiment, time of flight measurements could be translated to percentage changes in the acoustic velocity. Furthermore, the geometry of the surface was constant and consequently any changes in the reflected spectrum could be attributed to changes that occurred within the sample. 


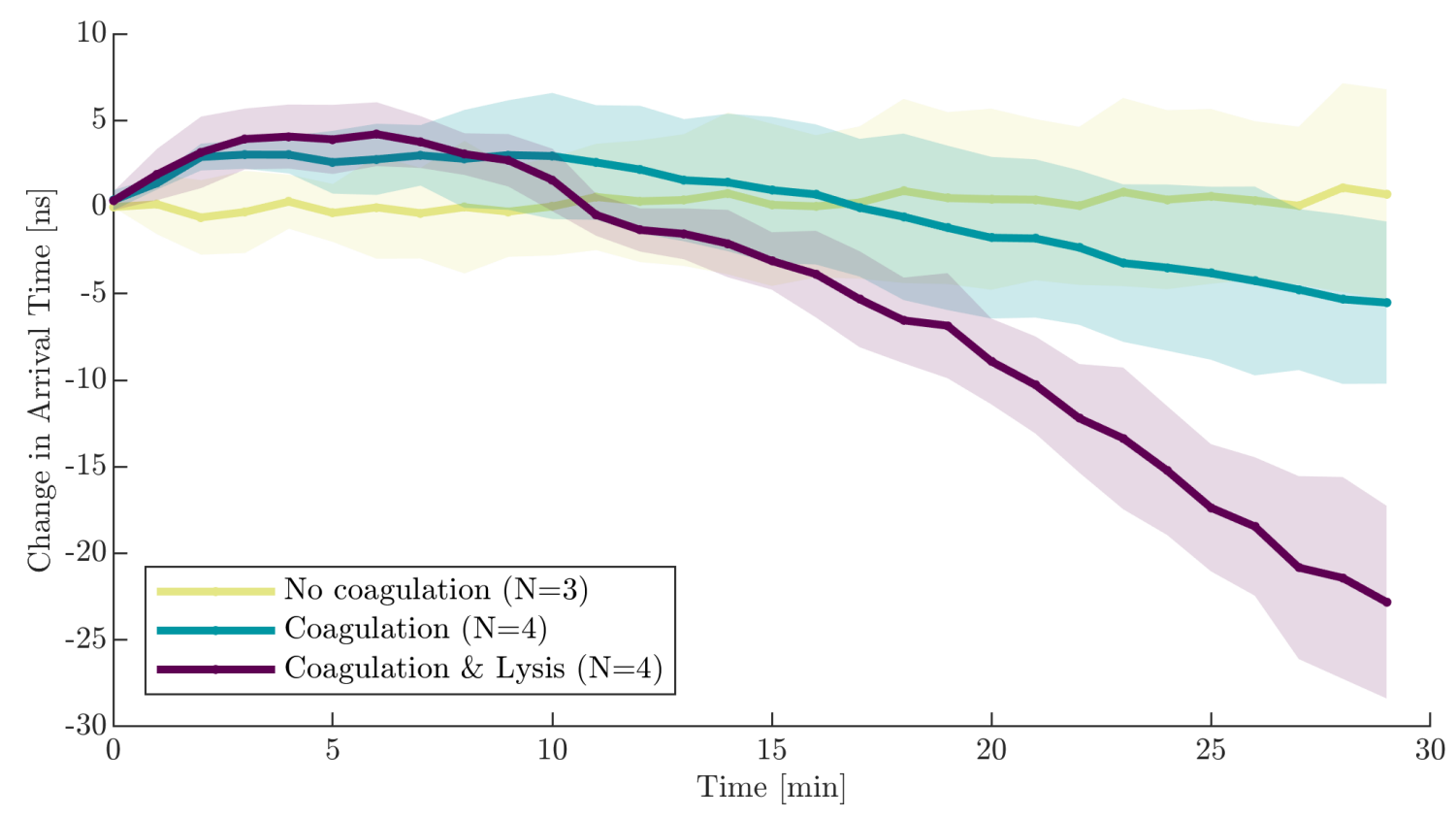

Figure 4.2: The mean and standard deviation in the arrival time data for control samples that don't undergo coagulation (yellow), samples that only undergo coagulation (green), and those that undergo both coagulation and lysis (purple). The ' $\mathrm{N}$ ' in the legend indicates how many experiments were used to calculate the mean and standard deviation for each sample type.

Three types of samples were prepared. The control sample of dilute plasma had no enzymes and, as a result, did not undergo any major biological changes. In the sample that underwent coagulation only, calcium chloride and thrombin were added. The final group, which underwent both coagulation and fibrinolysis contained $\mathrm{uPa}$ in addition to the calcium chloride and thrombin. All three samples were composed of $33 \%$ plasma.

Changes in acoustic velocity for the three sample groups are shown in Fig. 4.3. Measurements were repeated five times per sample type in an attempt to identify trends. In order to compare the changes to the measurements acquired before the cap was implemented, time of flight data that was used to calculate the percentage change in Fig. 4.3 is shown in Fig. 4.4. Representative reflection spectra can be seen in Fig. 4.5 for each sample type.

\subsection{Murine whole blood}

Compared to the plasma samples, whole blood contained cells that act as scatters to the incident US wave. In addition to analyzing the signal reflected off of the blood-cap interface, which provides information about the frequency dependent attenutation through the sample, information could be acquired by examining the back-scattered signal from within the blood 


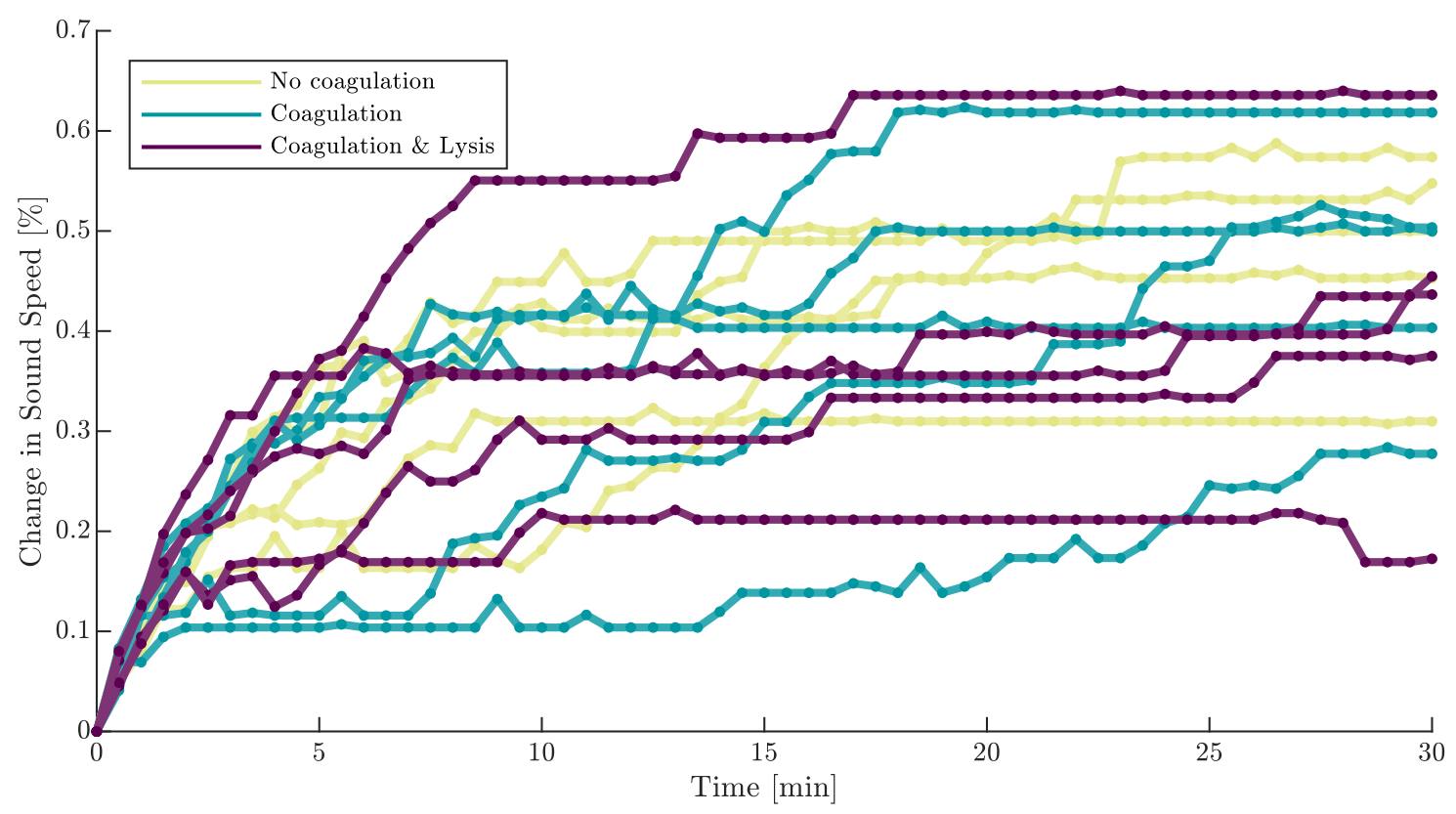

Figure 4.3: The percentage change in sound speed as a function of time in plasma for five control samples (no coagulation, yellow), five samples that undergo coagulation only (green), and five samples that undergo coagulation and fibrinolysis (purple).

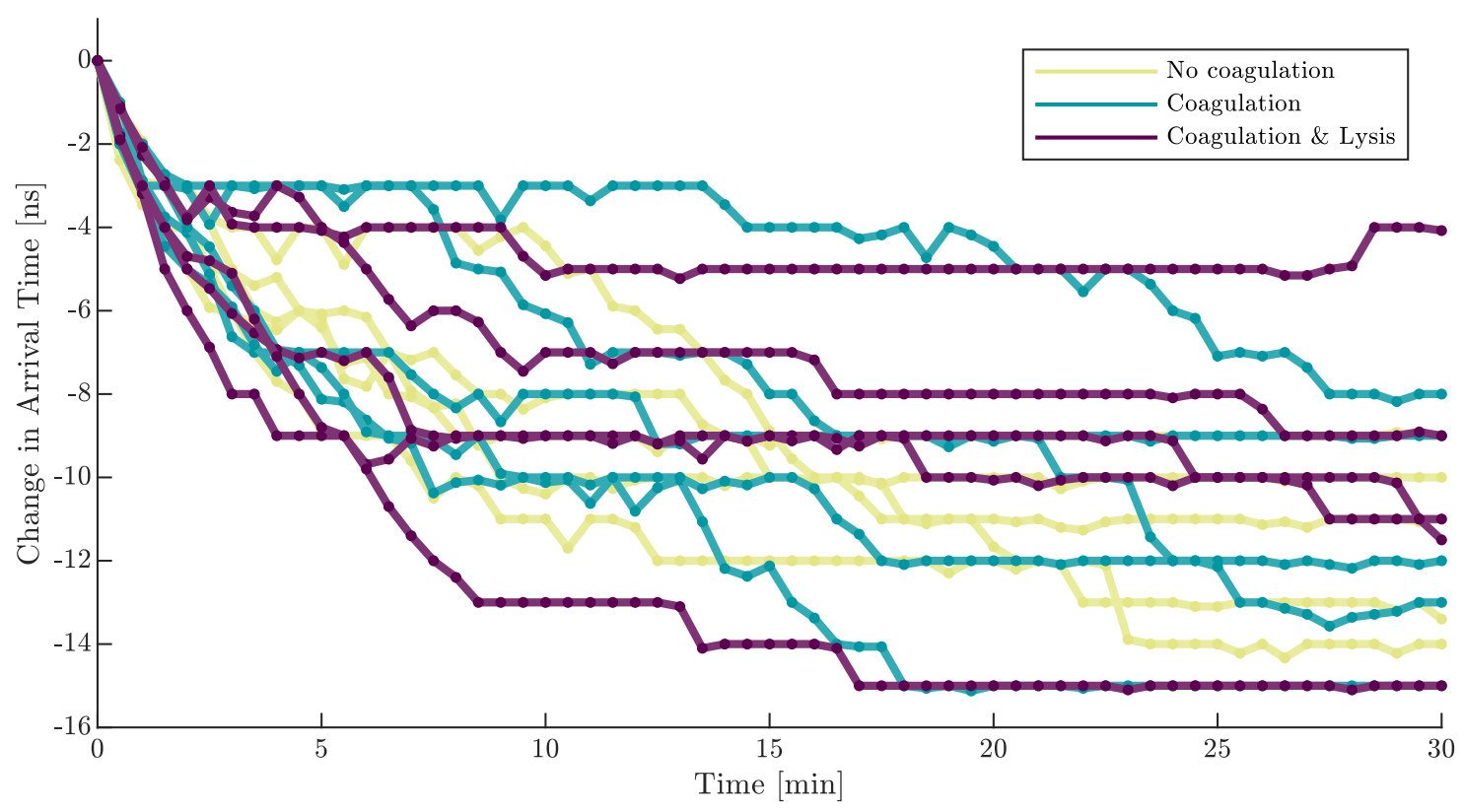

Figure 4.4: The change in arrival time in plasma for five control samples (no coagulation, yellow), five samples that undergo coagulation only (green), and five samples that undergo coagulation and fibrinolysis (purple). Data are from the same experiments as in Fig. 4.3. 
a)

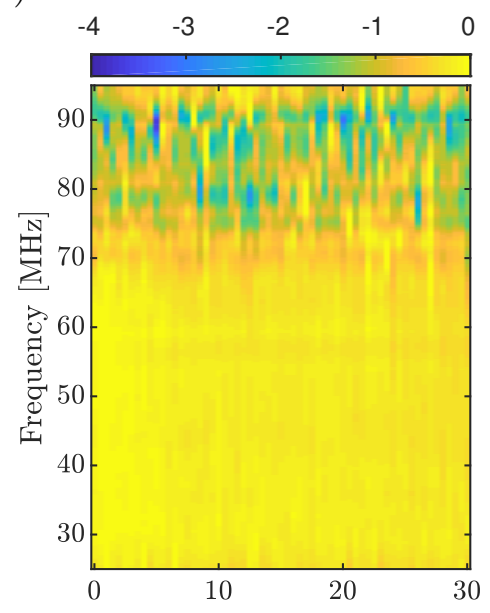

b)

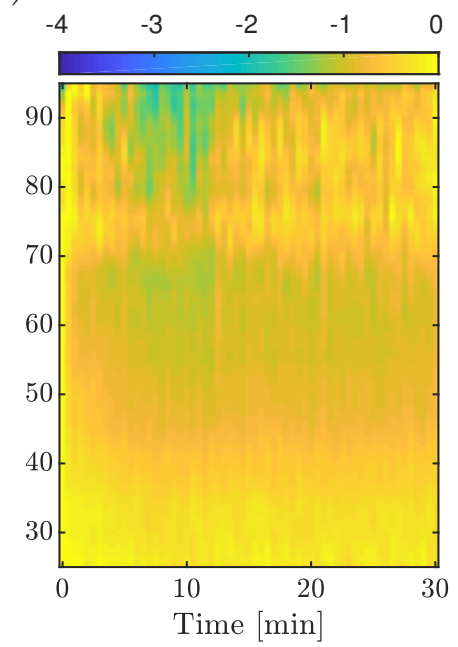

c)

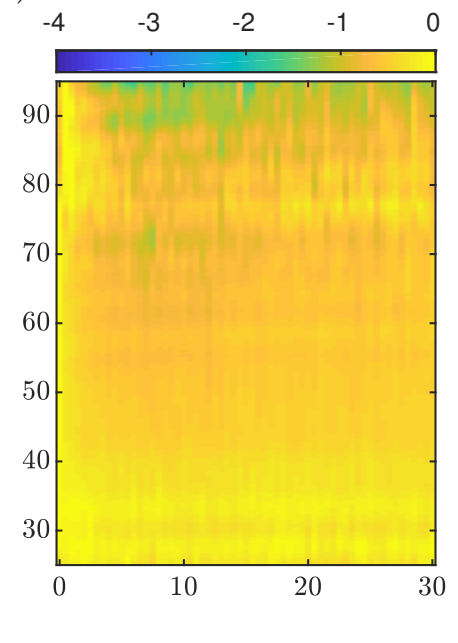

Figure 4.5: Representative frequency spectra from the reflection off of the cap-sample interface as a function of time in plasma for a) a control sample (no coagulation), b) a sample that only underwent coagulation, and c) a sample that underwent both coagulation and fibrinolysis. The colour scale represents $S$ in units of $\mathrm{dB}$.

region, which provides information about the size and motion of the US scatterers. A BMode image of the blood sample is shown in Fig. 4.6(a). A sample that has undergone partial retraction from the well's surface can be seen in Fig. 4.6(b). 
a)

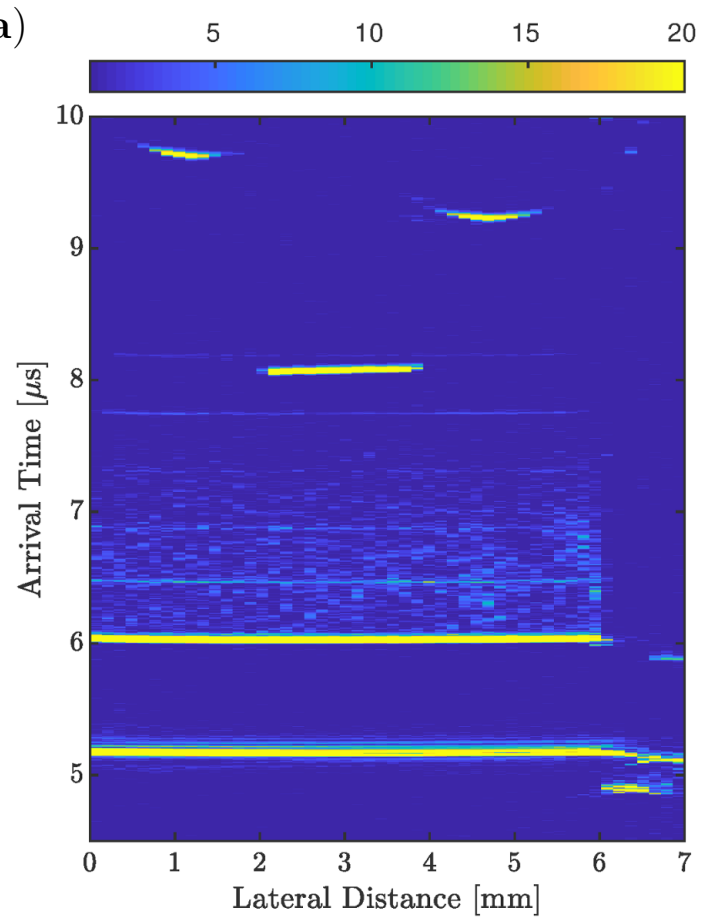

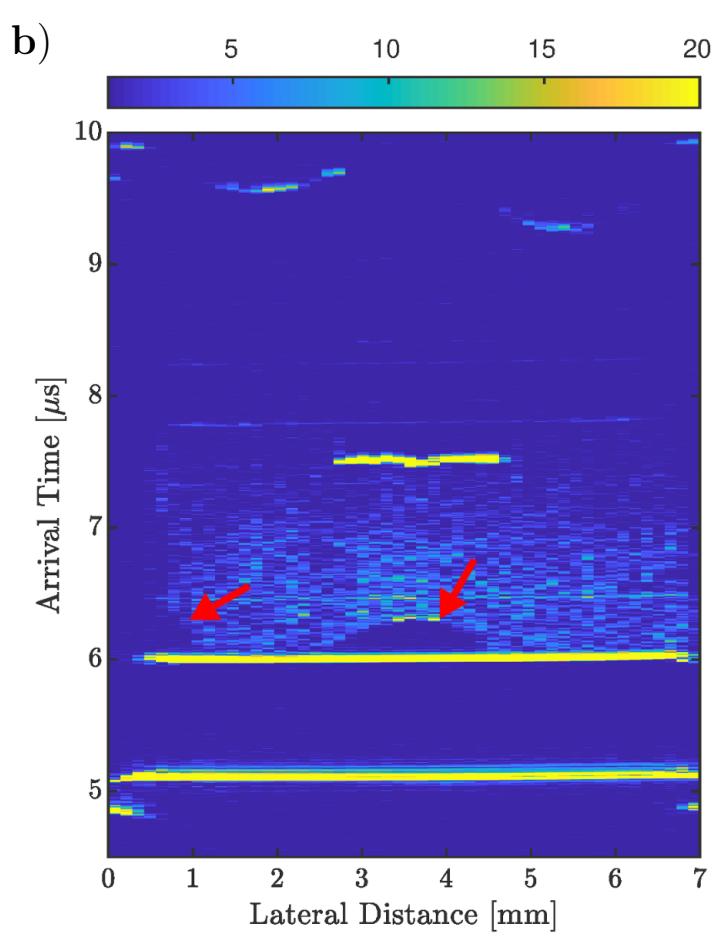

Figure 4.6: B-Mode images for two coagulated blood samples: (a) in the absence of clot retraction, and (b) with partial clot retraction (indicated by arrows). The bottom two interfaces correspond to the bottom of the well, the interface at the centre corresponds to the surface of the cap, and the two menisci from the sample-air interface can be seen at the top of the frame on either side of the cap. Colour scale is linear and was truncated at 20 a.u. for proper visualization of the blood backscatter.

\subsubsection{Acoustic Velocity}

Changes in acoustic velocity were calculated for 10-14 locations corresponding to the lateral region that contained the cap interface. Values were calculated using the time of flight between the bottom of the well and the surface of the cap as described in Sec. 3.1.5. An example of typical intra-sample variability across these locations is shown in Fig. 4.7. 


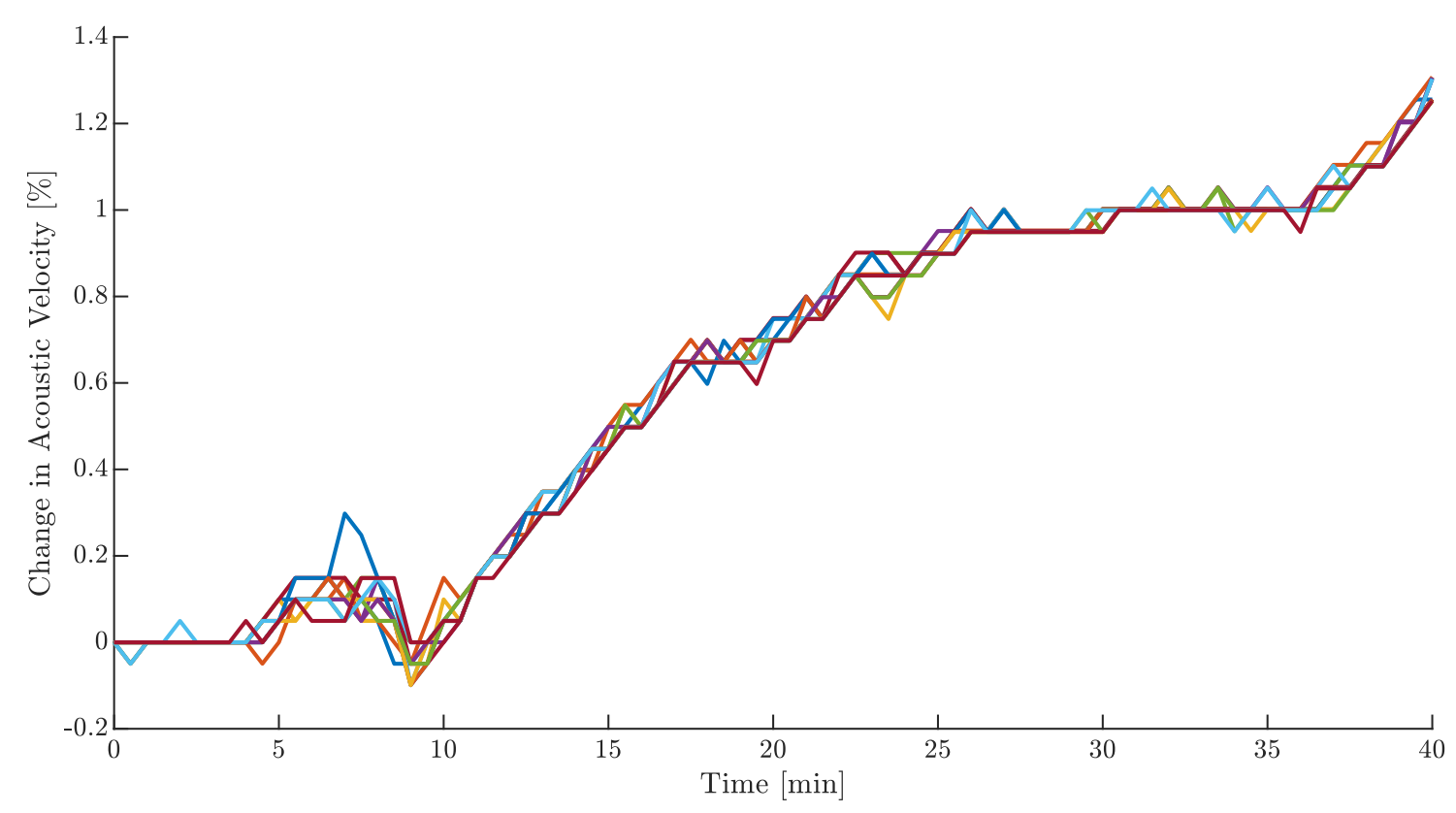

Figure 4.7: Intra-sample variation in acoustic velocity across 14 locations in $140 \mu \mathrm{m}$ increments for a CCM2 Flox recalcified blood sample.

Measurements were acquired for sixteen samples of recalcified citrated mouse blood across three strains (CD1, CCM2 Flox, C57). The results obtained for each strain can be seen in Fig. 4.8. Control measurements were acquired using partially dilute citrated blood. A comparison between the control and recalcified samples for CD1 mouse blood can be seen in Fig. 4.9.

a)

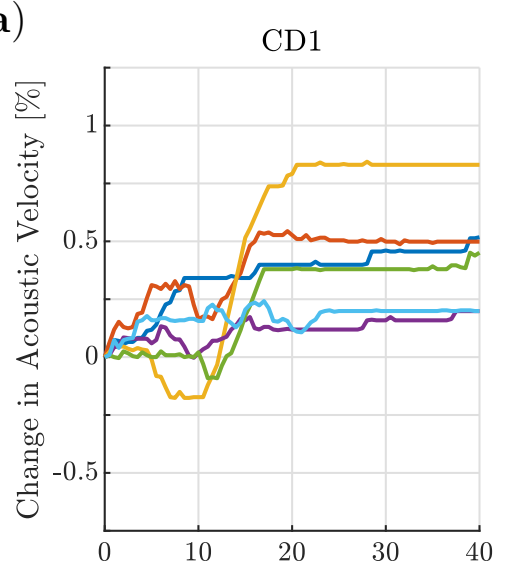

b)

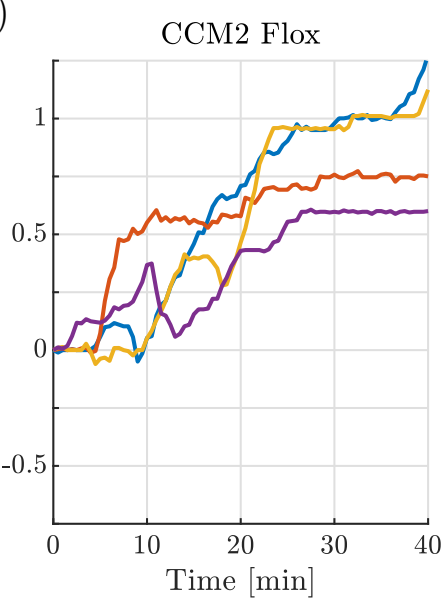

c)

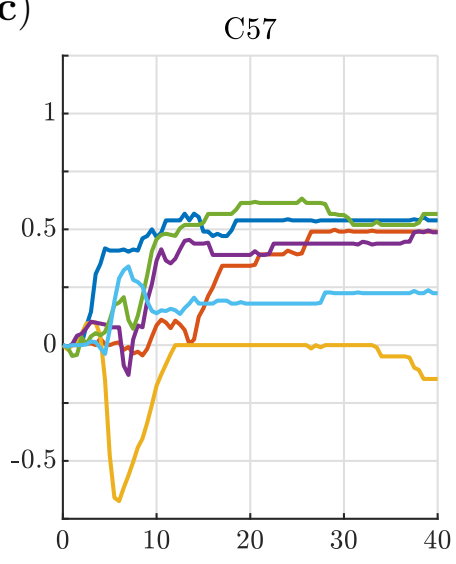

Figure 4.8: Change in acoustic velocity through recalcified blood samples, where each line represents the average of 10-14 measurements at different locations within the sample. Each plot is labelled according to the mouse strain with a) six CD1, b) four CCM2 Flox, and c) six C57 measurements. 


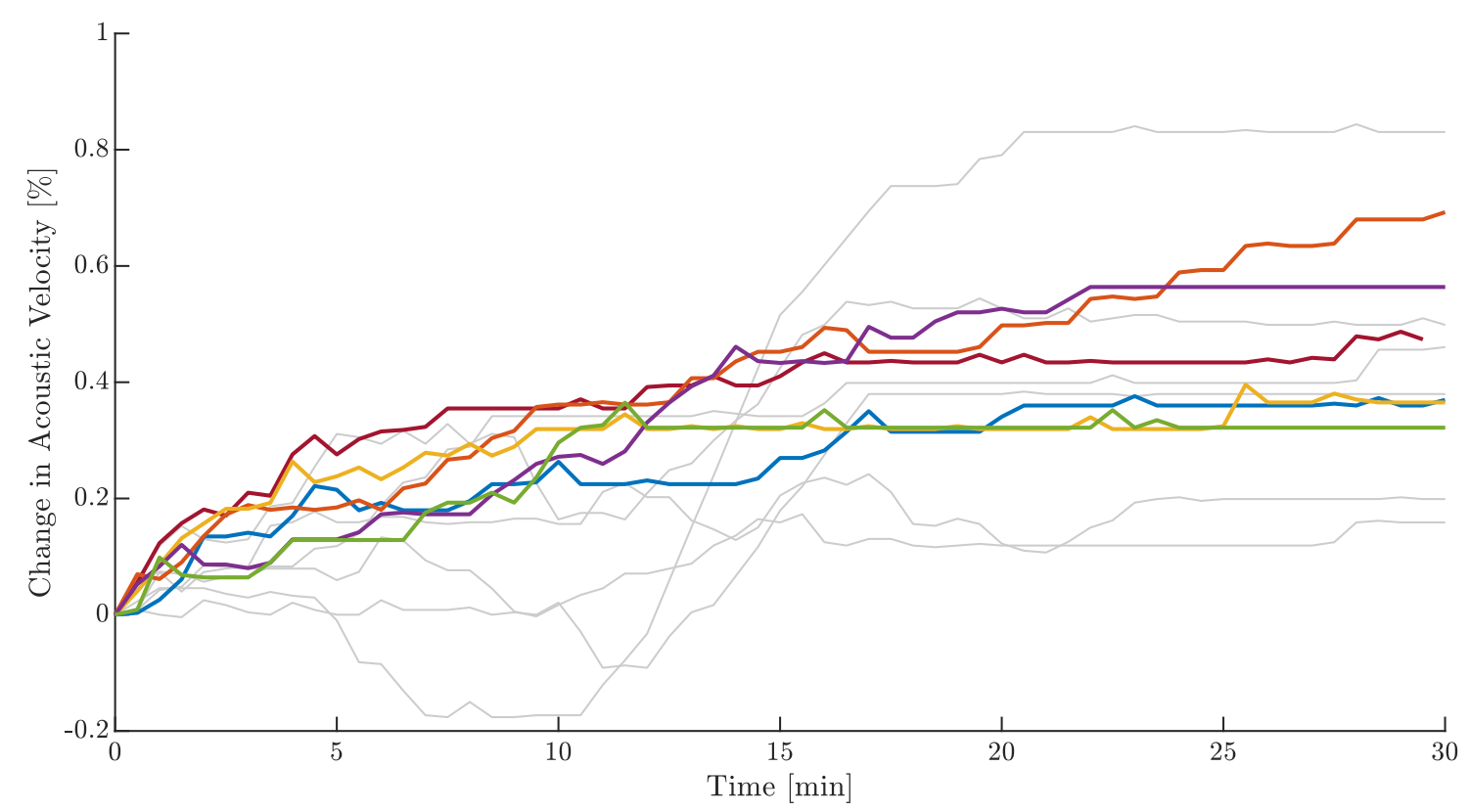

Figure 4.9: Change in acoustic velocity in six CD1 control samples are shown. The six recalcified CD1 samples that were shown in Fig. 4.8(a) are plotted in grey for comparative purposes.

\subsubsection{M-mode}

A comparison between two dilute blood samples and a recalcified blood sample from CD1 mice are shown in Fig. 4.10. Intra-sample variability can be observed in Fig. 4.11 for recalcified CD1 blood, which is in contrast to the intra-sample variation in the speed of sound (Fig. 4.7). Differences observed in certain regions of a subset of samples are shown in Fig. 4.12. In Fig. 4.12(a) clot retraction from the bottom of the well occurs in the region of interest, and is indicated by an arrow. In Fig. 4.12(b), a physical shift of the of the clot occurred (indicated by the arrow) as a result of clot retraction that was observed in the sample at other locations. Fig. 4.12(c) shows variation in the backscatter intensity near the centre of the clot. Fig. 4.12(d) appears to show incomplete/partial formation of the clot, an effect which is also visible in Fig. 4.12(b). 
a)

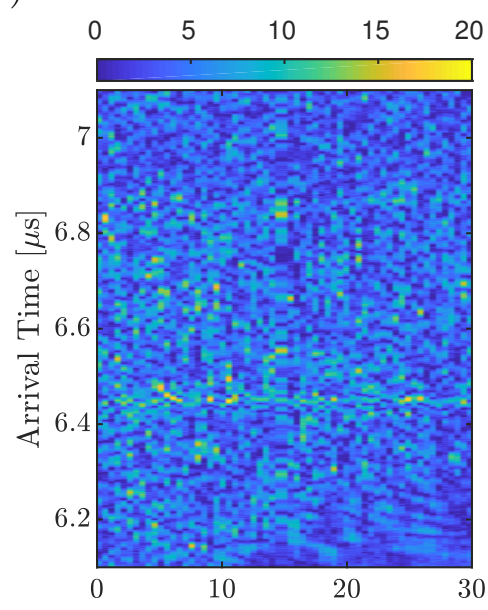

b)

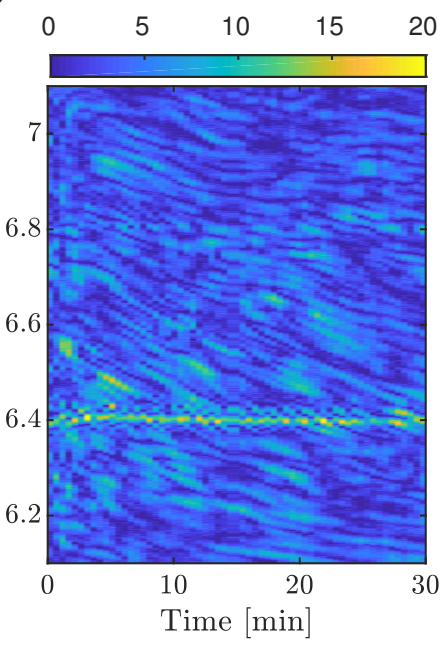

c)

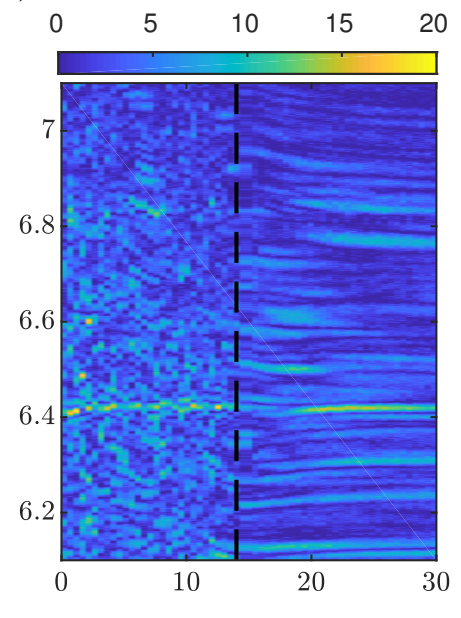

Figure 4.10: M-mode images from three CD1 samples: (a) 66\% dilute citrated blood, (b) 90\% dilute citrated blood, and (c) $94 \%$ recalcified citrated whole blood, where the dashed line indicates clot formation. The colour scale is linear [a.u.].

a)

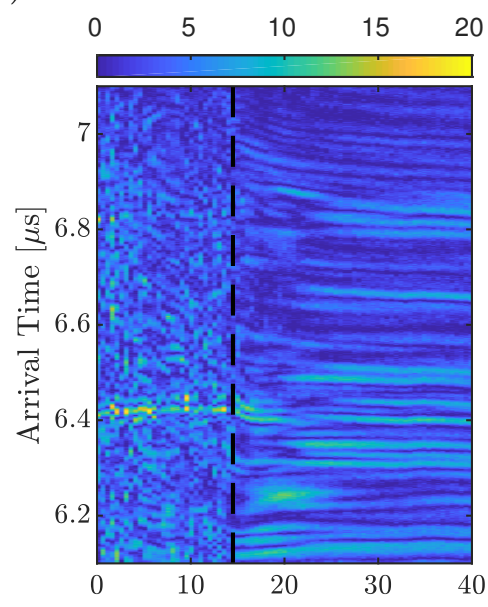

b)

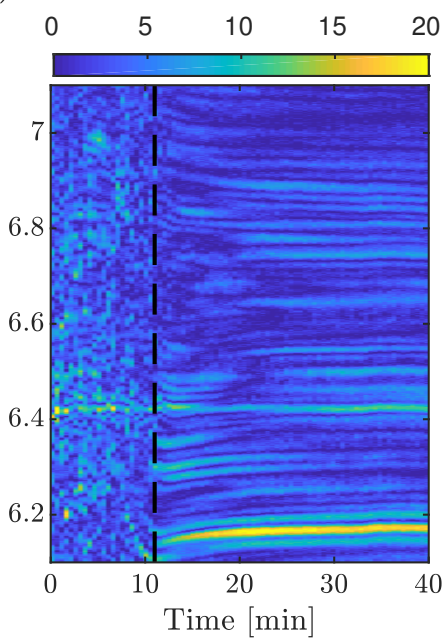

c)

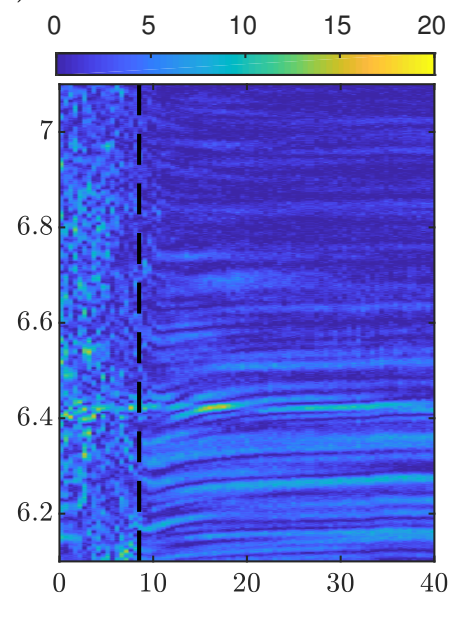

Figure 4.11: M-mode images for three locations separated by $420 \mu \mathrm{m}$ spacings in a sample of recalcified CD1 blood. The dashed lines represent the estimate of clot formation time. The colour scale is linear [a.u.]. 
a)

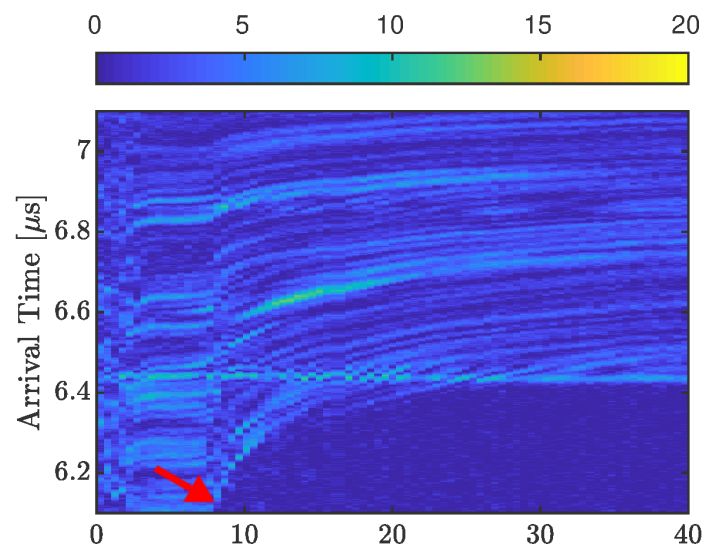

c)

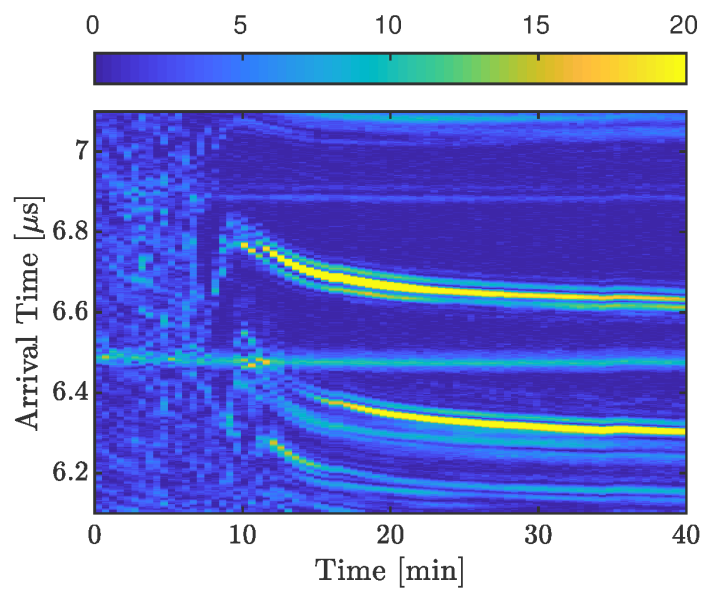

b)

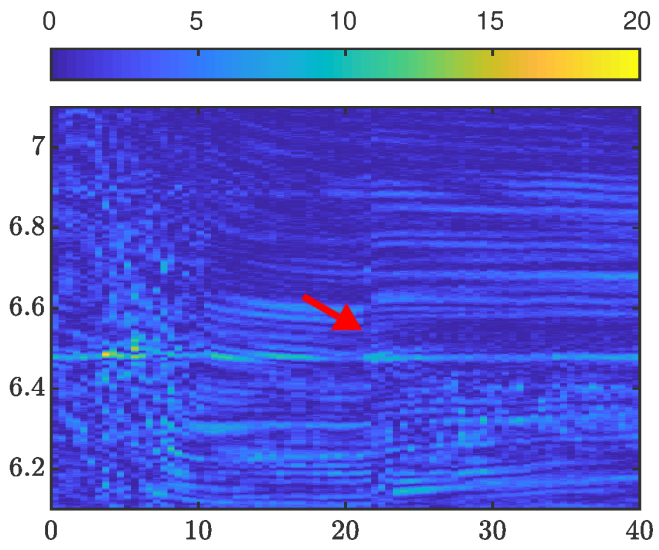

d)

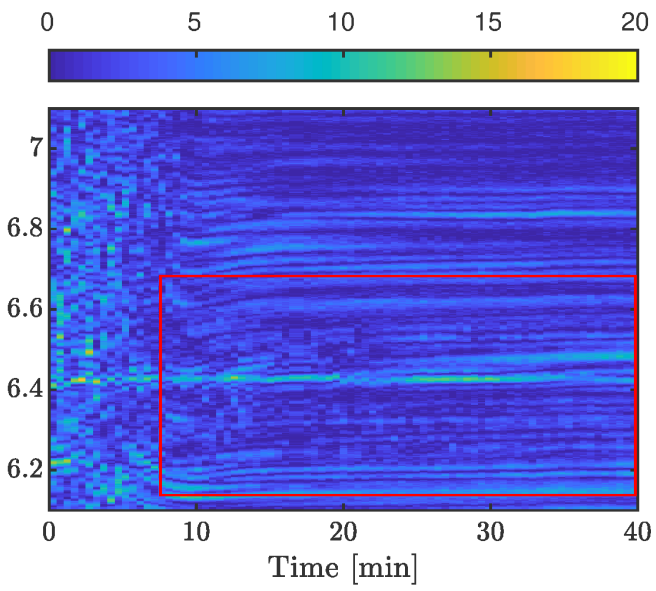

Figure 4.12: M-mode images from four different recalcified blood samples are shown to highlight variability in clot microstructure. Arrows indicate a) in-frame clot retraction and b) out-of-frame clot retraction. The red box in d) indicates regions with fluctuations, possibly corresponding to incomplete/partial formation. The colour scale is linear [a.u.].

\subsubsection{Reflection spectra}

The frequency spectra of pulses reflected from the sample-cap interface were acquired as described in Sec. 3.1.5. Spectra corresponding to the same samples and RF-lines as the M-mode images shown in Sec. 4.2.2 are shown in Figures 4.13, 4.14 and 4.15. The colour scales for all reflection spectra are displayed in $\mathrm{dB}$. 
a)

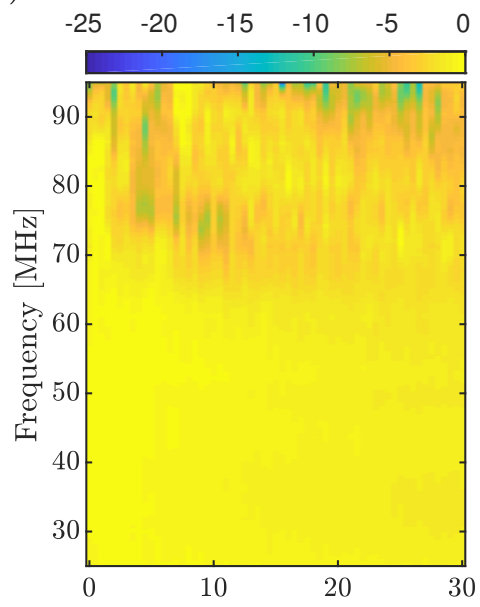

b)

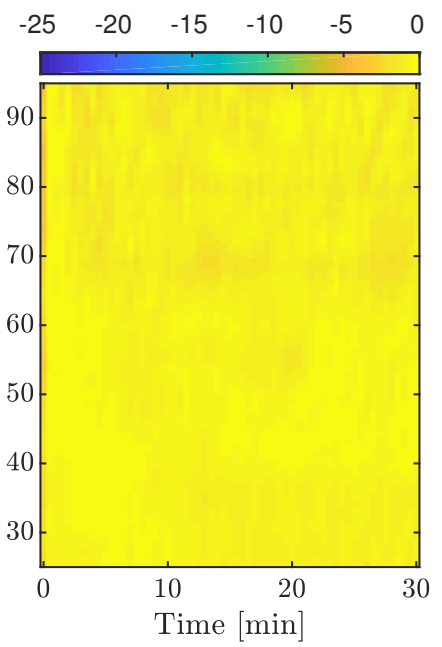

c)

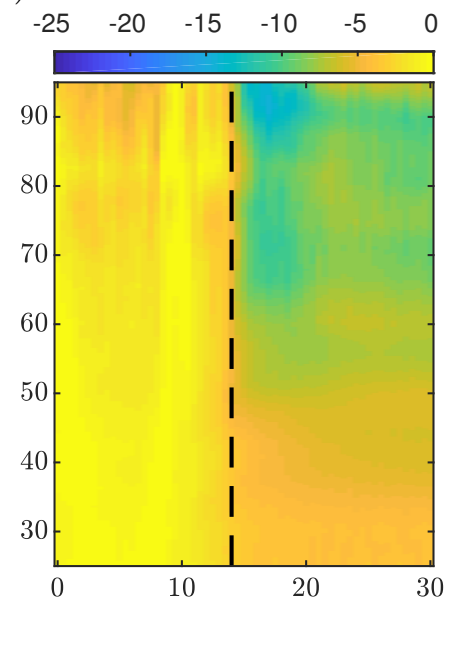

Figure 4.13: Normalized reflection frequency spectra from the three CD1 samples in Fig. 4.10 are shown, where (a) is $66 \%$ dilute citrated blood, (b) is $90 \%$ dilute citrated blood and (c) is $94 \%$ recalcified citrated whole blood. The colour scale represents $S[\mathrm{~dB}]$.

a)

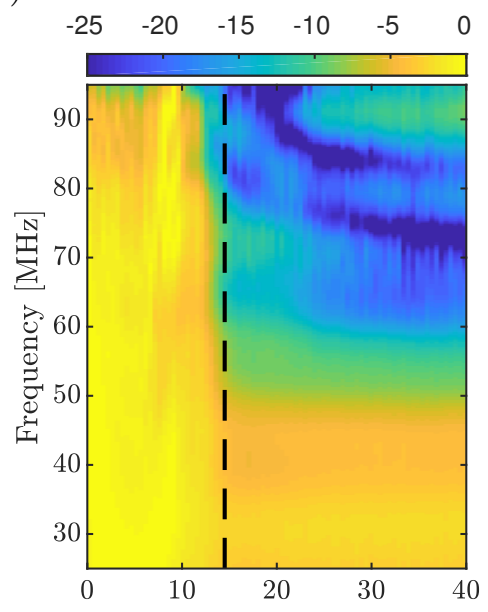

b)

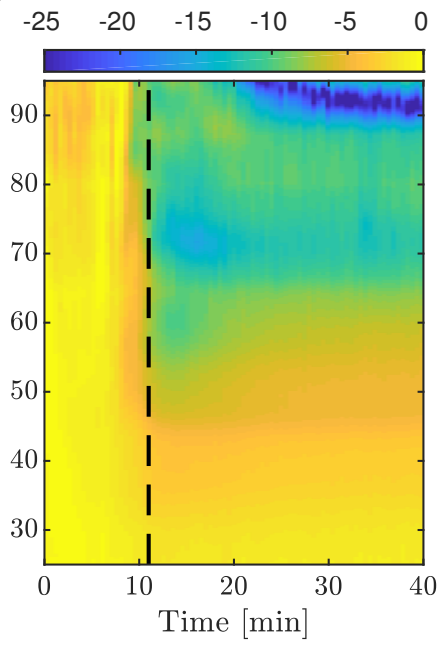

c)

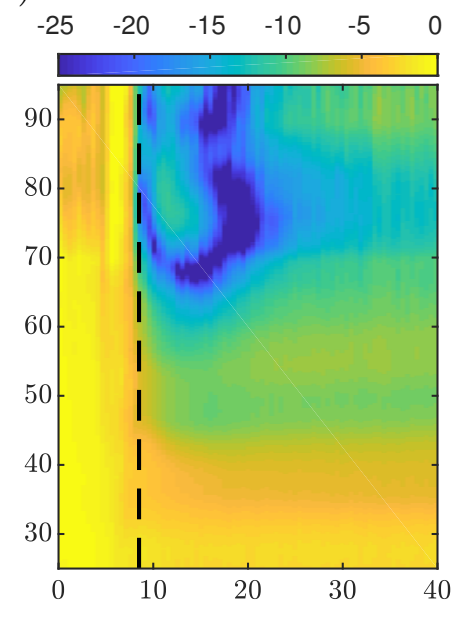

Figure 4.14: Normalized reflection frequency spectra are shown for three locations separated by $420 \mu \mathrm{m}$ in a sample of recalcified CD1 whole blood. The dashed lines estimate clot formation time based on the M-mode images shown in Fig. 4.11. The colour scale represents $S[\mathrm{~dB}]$. 
a)

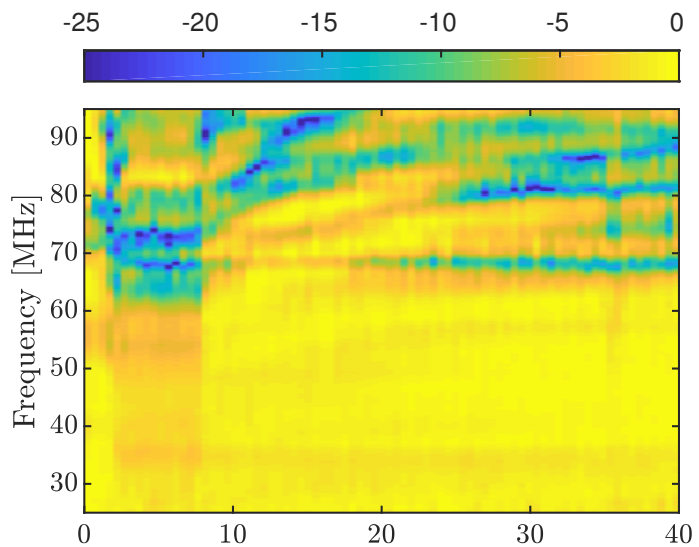

c)

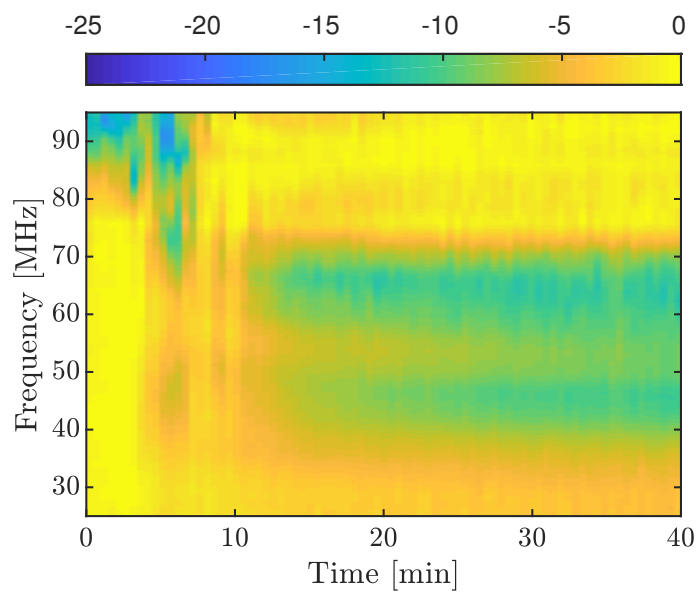

b)

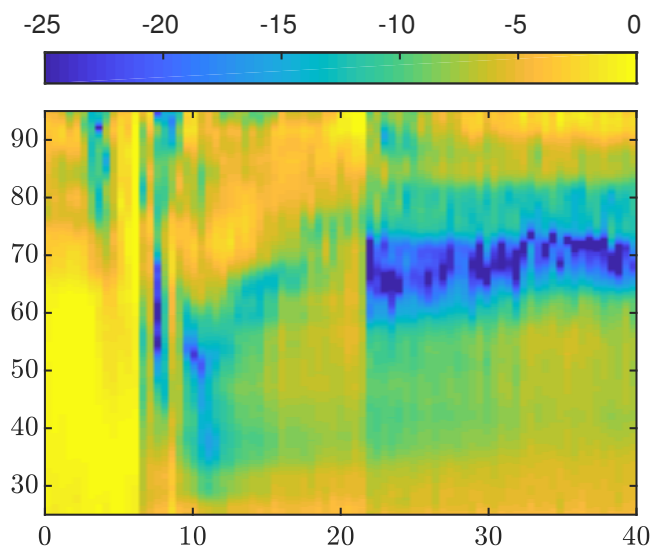

d)

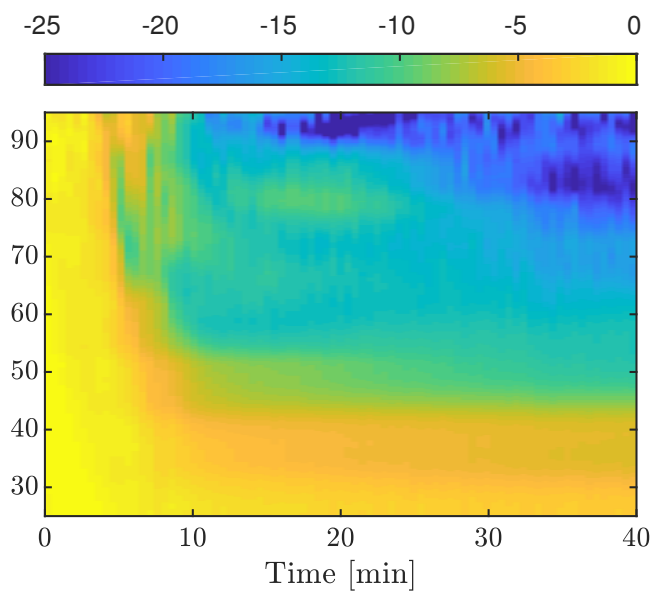

Figure 4.15: Normalized reflection frequency spectra from the four recalcified blood samples in Fig. 4.12 are shown. The colour scale represents $S[\mathrm{~dB}]$.

\subsubsection{Comparisons}

In order to combine the information provided by the change in acoustic velocity, M-mode images and reflection spectra data, compilations of the previously shown results have been constructed with a few modifications. Each combined figure displays the reflected frequency spectrum first, followed by the M-mode image, and then the acoustic velocity. Colour bars for the reflection spectra and M-mode images are omitted for simplicity. However, the scales are the same as those presented in Sec. 4.2.3 and Sec. 4.2.2 respectively, with frequency spectra presented as $S$ over a range of -25 to $0[\mathrm{~dB}]$ (logarithmic) and M-Mode images presented on a linear scale from 0 to 20 [a.u.]. Overlayed onto the M-mode image is a line plot of the frequency changes at $80 \mathrm{MHz}$, used to illustrate time dependant changes in the reflected 
frequency spectra. The corresponding frequency axes are in $\mathrm{dB}$ and can be found on the right side of each M-mode image. Finally, the acoustic velocity changes that were calculated for the sample are shown. The changes corresponding to the A-Mode frame displayed in the other modalities will appear in colour, while the remaining locations will appear in grey to highlight the variation observed in that particular sample.

The summary shown in Fig. 4.16 combines the information given in Figures 4.10 and 4.13. Fig. 4.17 combines the results from Figures 4.11 and 4.14, and Figures 4.18 and 4.19 combine the results from Figures 4.12 and 4.15 .

a)
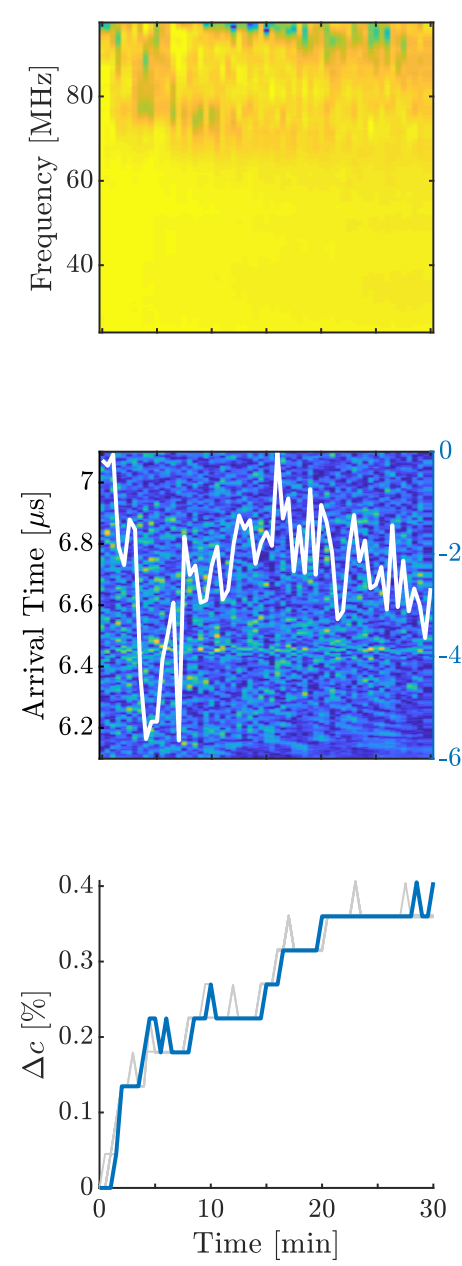

b)
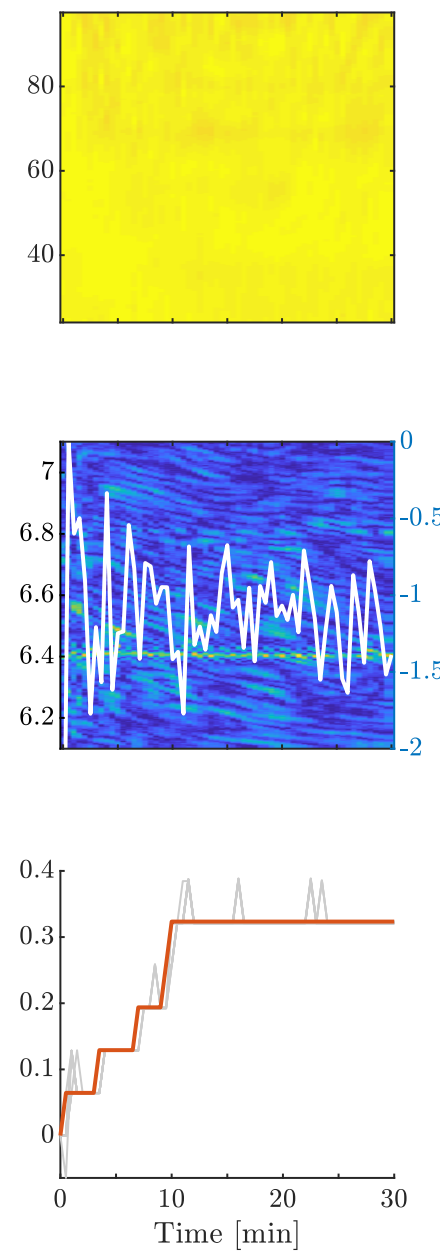

c)
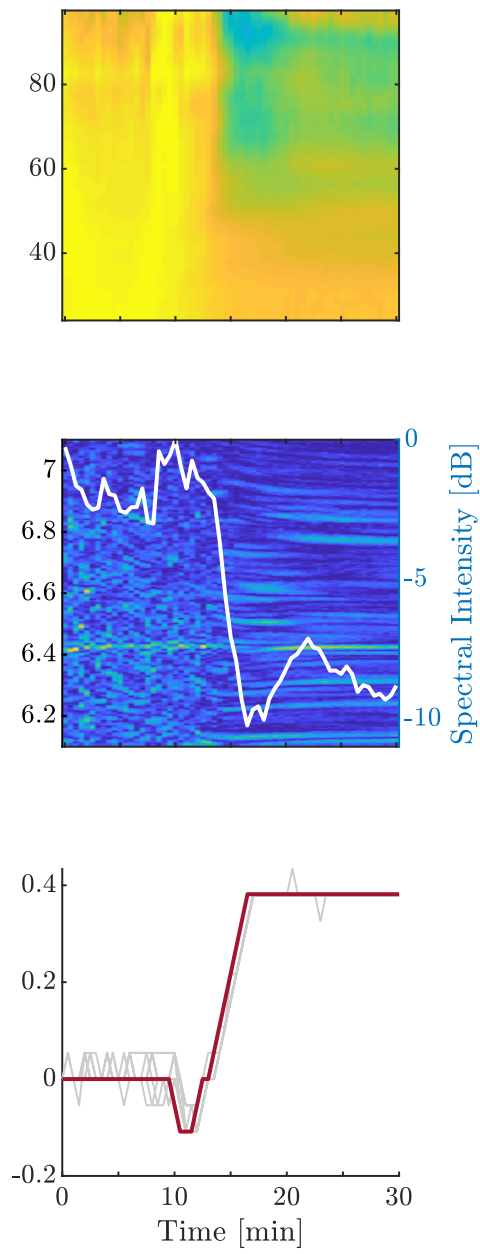

Figure 4.16: A comparison between Figures 4.10 and 4.13, along with the acoustic velocity change for each sample location. The acoustic velocity at the locations in the M-mode and reflected frequency data is in colour, while the acoustic velocity at other locations in the same sample is in grey. a) a $66 \%$ dilute whole blood sample, b) a $90 \%$ dilute whole blood sample, and c) a $94 \%$ recalcified sample that undergoes coagulation. 
a)
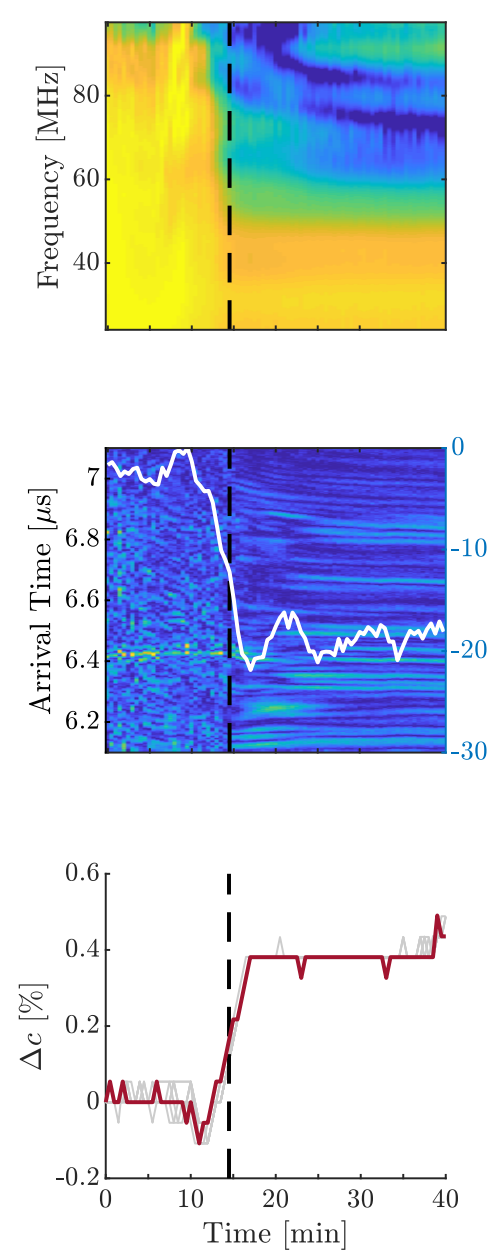

b)
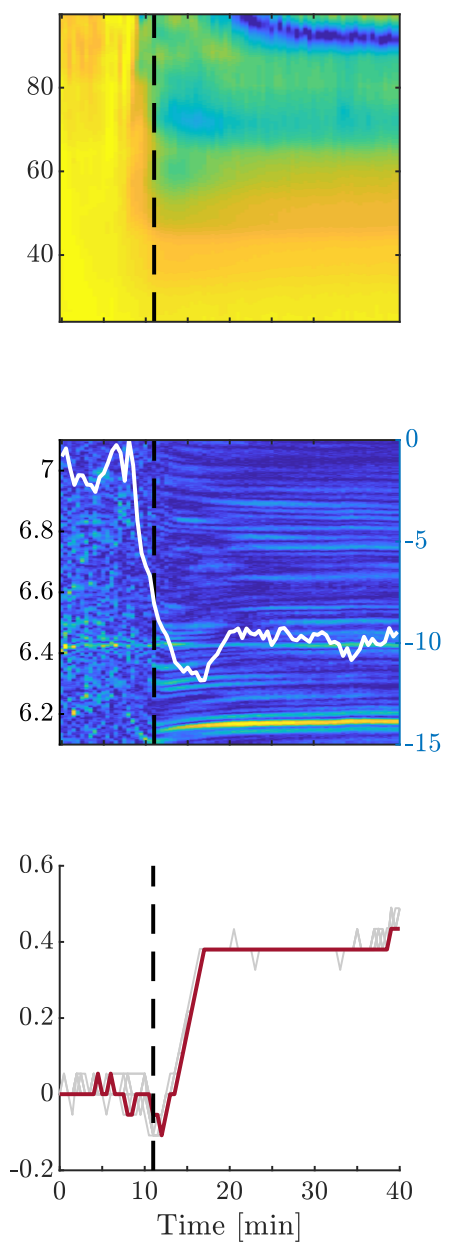

c)
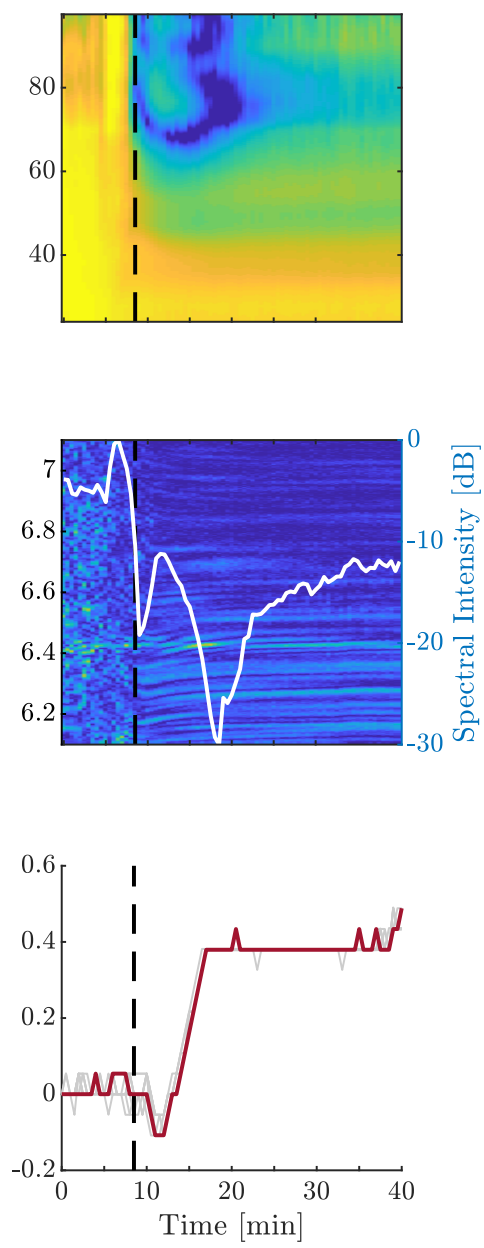

Figure 4.17: A comparison between Figures 4.11 and 4.14, along with the acoustic velocity change for each sample location. The acoustic velocity at the locations in the M-mode and reflected frequency data is in colour, while the acoustic velocity at other locations in the same sample is in grey. The data are for three locations separated by $420 \mu \mathrm{m}$ spacings in a sample of recalcified CD1 blood. 
a)
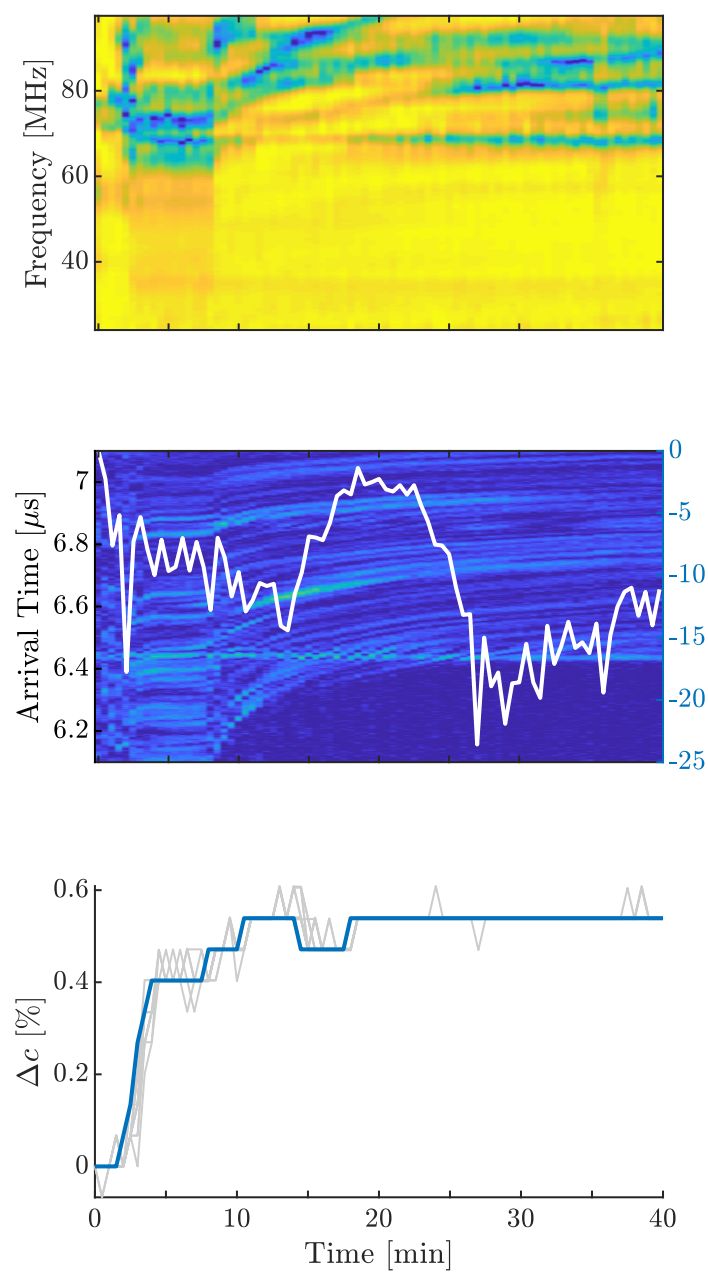

b)
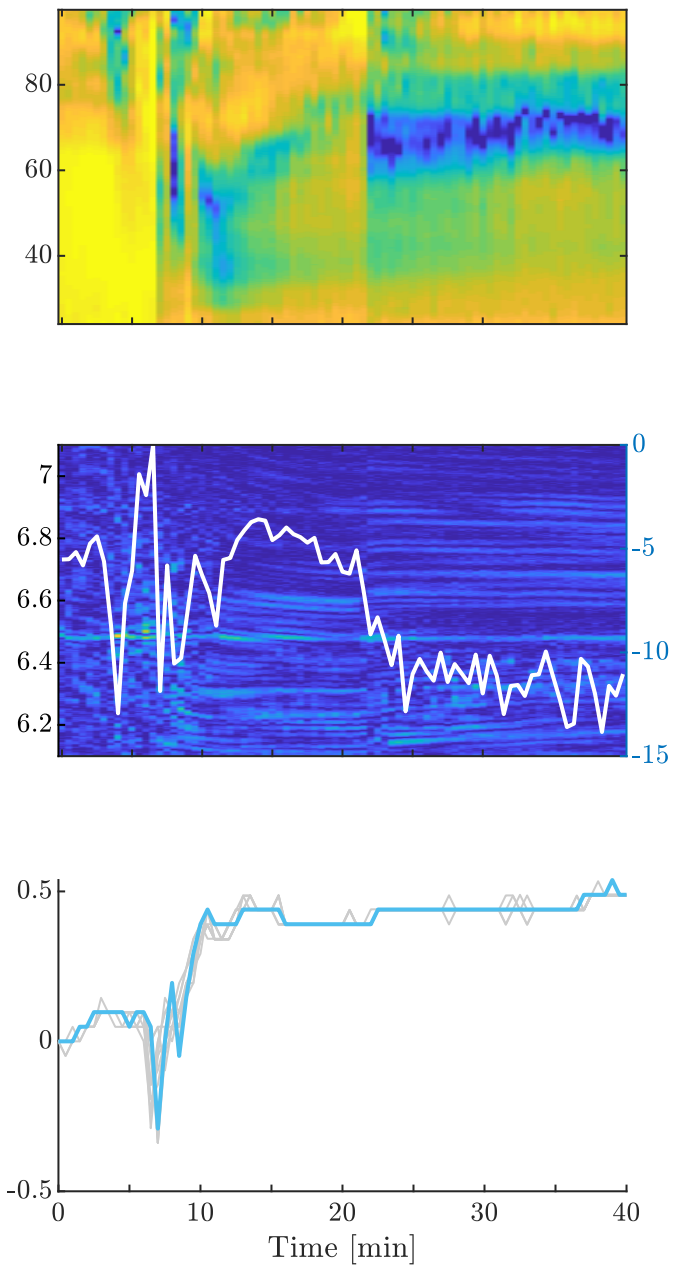

Figure 4.18: Comparisons between a) the data presented in Figures 4.12(a) and 4.15(a), and b) the data presented in Figures 4.12(b) and 4.15(b). The acoustic velocity at the locations in the M-mode and reflected frequency data is in colour, while the acoustic velocity at other locations in the same sample is in grey. Clot retraction is shown to affect the data when a) it lies along the acquisition line, and b) when it occurs out of the frame. 
a)
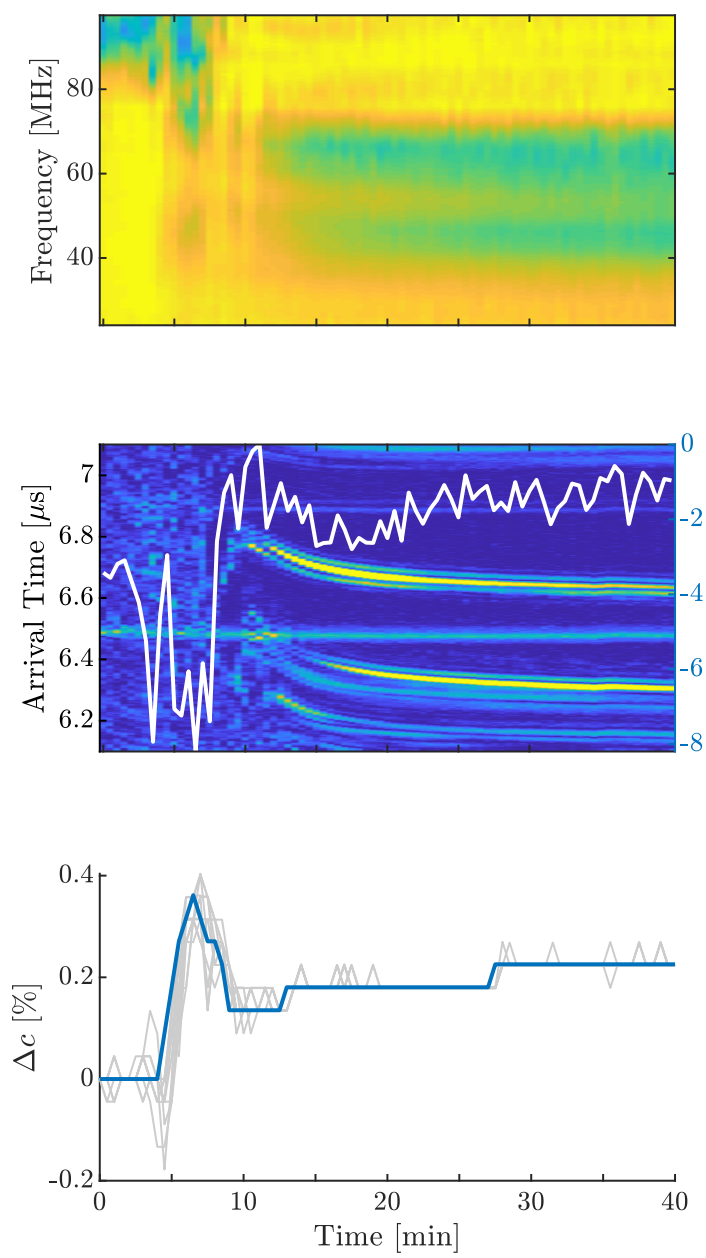

b)
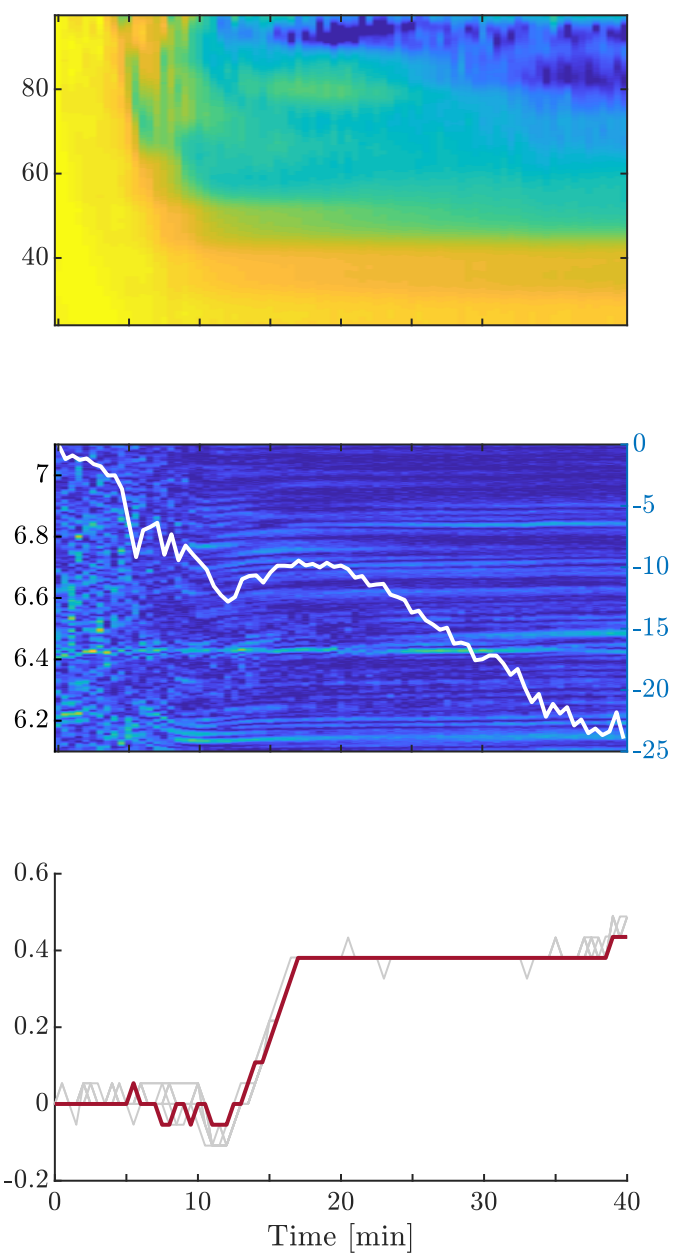

Figure 4.19: Comparisons between a) the data presented in Figures 4.12(c) and 4.15(c), and b) the data presented in Figures $4.12(\mathrm{~d})$ and $4.15(\mathrm{~d})$. The acoustic velocity at the locations in the M-mode and reflected frequency data is in colour, while the acoustic velocity at other locations in the same sample is in grey. This highlights how each metric is affected by a) regions without backscatter that appear in the centre of the sample, and b) what is hypothesized to be incomplete clot formation. 


\section{Chapter 5}

\section{Discussion}

In this chapter, the results presented in Sec. 4 are discussed. This starts with the plasma experiments, where the impact of the plug on speed of sound measurements is highlighted, and potential explanations for the observed trends are considered. Next, the insights into clot kinetics gained from each metrics used to assess the whole blood coagulation are exam-

ined. How these metrics can be used alongside one another to obtain a more comprehensive understanding of clot kinetics is then discussed.

\subsection{Human Blood Plasma}

The experiments performed using human blood plasma were designed to emulate the sample preparation used for the OHP tests. This was achieved by using the 96-well microtitre plates with a total volume of $100 \mu \mathrm{L}$ and comparable plasma and enzyme concentrations.

In the initial experiments, the constituents were combined in a well and the system was sealed with an adhesive strip to minimize evaporation. The B-Mode image corresponding to the original setup is shown in Fig. 4.1(a), where the top surface of the sample can be seen as a curved line near the top of the image. The curvature of the meniscus caused sound waves closer to the edges of the well to be deflected away from the transducer, which resulted in no reflections recorded outside of the central region. In later iterations, the well cap was introduced, which provided a flat $2 \mathrm{~mm}$ surface at the centre of the well from which the US waves were reflected. This can be seen in Fig. 4.1(b), where the menisci on either side of the plug are also visible.

\subsubsection{Before the well cap addition}

Without the well cap, the time of flight between the bottom of the well and the surface of the sample was subject to changes both as a result of changes in the acoustic velocity, as well as to changes in the physical location of the surface. The changes in time of flight recorded for the three plasma sample types are shown in Fig. 4.2. A number of trends appear to be visible. Firstly, the sample without coagulation is stable, with its mean centred about zero and a relatively consistent standard deviation throughout the experiment. In the two 
sample types that underwent coagulation, a consistent increase in the time of flight of several nanoseconds was observed in the first five minutes, followed by a relatively linear decrease afterwards. The samples that underwent lysis (where the u-PA concentration was titrated to lyse the sample in $\sim 20$ minutes) had a steeper slope than the samples whose clots reamined intact.

While there appeared to be clear differences between the various sample types, the changes were relatively small given the fact that the system was set to detect changes in 1 ns increments. Additionally, it was unclear whether the observed changes in time of flight occurred as a result of changes in the acoustic velocity (due to coagulation or changes in temperature) or changes in the distance travelled by the US wave (due to changes in the location of the meniscus caused by evaporation or volume change due to clot formation). In order to isolate these two variables, the well cap was developed to fix the distance travelled by the wave.

\subsubsection{After the well cap addition}

With the well cap, percentage changes in acoustic velocity were calculated for the three plasma sample types. These are shown in Fig. 4.3. From the data, all samples experienced an increase in the acoustic velocity ranging from $\sim 0.2 \%$ to $0.6 \%$. There was a significant overlap in all sample types and there did not appear to be any distinguishing features between them. These changes were on the order of the changes expected for a temperature increase from $33^{\circ} \mathrm{C}$ to $37^{\circ} \mathrm{C}$ as discussed in Sec. 2.3.2. Changes of this size were reasonable to expect despite the warming of the plasma. This is because the the sample constituents must be combined outside of the $37^{\circ} \mathrm{C}$ chamber, which can take anywhere between 30 and 60 seconds. These results appear to confirm the findings of Plag et al., who reported that changes in the acoustic velocity in clotting plasma are not significantly different from those that do not undergo clot formation [69]. The results shown here add to previous research by also showing that there were no significant changes in the acoustic velocity as a result of clot breakdown.

The reflection spectra for the three samples obtained from the reflection off the well cap are shown in Fig. 4.5. A minor drop in the signal intensity at higher frequencies (70$90 \mathrm{MHz}$ ) was observed following the first time points in samples that underwent coagulation. However, these changes were small (on the order of $1 \mathrm{~dB}$ ) and they generally occurred within a minute of starting the data acquisition. This made it difficult to determine whether the changes corresponded to stages of the coagulation process or to fluctuations in the data unrelated to the coagulation process. In order to properly assess whether or not the signal 
intensity changed as a result of the coagulation, it is likely that these spectra would need to be acquired with a higher temporal resolution than the 30 second intervals used here.

\subsubsection{Comparison of results}

In order to compare the data with and without the well cap, the results shown in Fig. 4.3 have been presented in terms of change in time of flight (Fig. 4.4). Given that the acoustic velocity changes resulted in a decrease in arrival time, the trends shown in Fig. 4.2 are likely attributed to changes in the surface height of the clot. It is hypothesized that the increase observed in the first several minutes in the coagulating samples were a result of changes in the geometry of the meniscus caused by fibrin polymerization. As the mesh formed and began to contract, it is possible that the meniscus flattened. This would have resulted in an increase in the distance travelled by the wave near the centre of the well and therefore an increase in the arrival time.

The decreases in arrival times observed beyond the 10 minute mark seemed to occur once the velocity changes had mostly stabilized, and only for samples with coagulation. This indicates that the decreases were a result of decreased distance travelled by the wave. The changes are roughly on the order of what would be expected due to evaporation. In water, the expected change in arrival time over a 30 minute period is on the order of $20 \mathrm{~ns}$ when an adhesive strip is used to seal the well. It is possible that the sample that underwent fibrinolysis saw a more rapid decrease in the arrival time simply due to the fact that it is liquid and therefore evaporates more quickly. Further investigation is necessary to confirm the reasons behind the trends observed without the well cap. However, due to the geometric basis of the changes, it is unlikely that the time of flight results provide reliable insight into the biochemical changes occurring within the medium.

\subsection{Murine whole blood}

In the context of this work, constructed B-Mode images provide little quantitative information. They do, however, act as an effective way to visualize the system's geometry and summarize signal information from fifty RF-lines in a single image. This proved particularly useful in identifying regions of interest, mainly in identifying the location of the plug laterally as well as axially for each experiment.

For coagulating whole blood, B-Mode images can also highlight structural changes that occur within the sample. Fig. 4.6 illustrates such a situation, where the sample underwent retraction from the vessel walls. While clot retraction did not occur to the same extent in all 
samples, it is a worthwhile effect to keep note of as it can impact measurements, especially ones that measure attenuation of the US through the medium.

\subsubsection{Acoustic velocity}

\section{Intra-sample variability}

A change in acoustic velocity can indicate fundamental changes in the medium through which the acoustic wave propagates. Presented in this work as the percentage change relative to the initial time point, measurements were acquired at ten to fourteen locations for each sample based on time of flight information. Fig. 4.7 shows the consistency of acoustic velocity change across all locations in a given sample. This was true for all samples across all mouse strains and regardless of whether or not the sample underwent coagulation. This was to be expected under the assumption that the sample was relatively homogeneous. However, as will be discussed in Sections 5.2.2 and 5.2.3, M-mode and reflected spectral data seem to indicate that this is not the case. Inhomogeneities observed within the sample using the other modalities (M-mode, reflected spectrum) do not give rise to significant changes in the acoustic velocity. As such, the acoustic velocity should not be used to evaluate intra-sample variability.

\section{Inter-sample variability}

Despite the high degree of consistency observed within a sample, significant variability was observed when comparing across different samples (Fig. 4.9). Given the sensitivity of acoustic velocity to physical parameters such as temperature, it is likely that at least some of these differences arise simply as a result of minor discrepancies relating to the sample preparation. The changes observed were generally positive and for the majority of samples did not exceed $0.5 \%$. This lies within the predicted range for temperature increases on the order of several degrees [78]. However, temperature equilibration is a consistent and gradual process that generally stabilizes within the first several minutes of acquisition. Given the significant changes that occurred ten or even twenty minutes into the certain experiments, at least a portion of these changes must occur due to the complex biological transitions occurring within the sample.

Comparing the results shown in Fig. 4.9 to results obtained by Callé et al. [16] in human and in rat blood (see Fig. 2.9), the characteristic sigmoidal shape was only present in a small subset of the samples. In the cases where the trends appeared to be similar, the extent of the changes observed in this work were less than those reported in the literature. If the mouse blood used for this work is assumed to transmit mechanical waves in a similar manner as rat 
blood based on the fact that both species' erythrocytes have diameters and mean corpuscular volumes that lie within the same range [52], the acoustic velocity in a mouse blood sample is approximately $1574 \mathrm{~m} / \mathrm{s}$ at $37^{\circ} \mathrm{C}$. This indicates that the maximum observed change due to coagulation over a forty minute period corresponded to an increase in acoustic velocity of $20.6 \mathrm{~m} / \mathrm{s}$, with an average change of $8.6 \mathrm{~m} / \mathrm{s}$. Box plots representing the distribution of predicted final velocities across each of the mouse strains are shown in Fig. 5.1. Although the largest change approached a published value of 1600-1606 m/s observed in coagulated rat blood, the majority of the samples exhibited changes much smaller than this. It is possible that this is due to differences in rat and mouse blood, and their coagulation kinetics.

Further discussion relating to how the acoustic velocity matches trends in the M-mode and frequency domain of the reflected pulse can be found in Sec. 5.2.4. These comparisons highlight a certain amount of correlation between the acoustic velocity measurements and the perceived clot kinetics revealed by the other analysis techniques. However, the acoustic velocity alone cannot be used to assess whether or not a sample has undergone coagulation. For one, the data across samples is highly variable and identifying the point at which coagulation occurs is not possible for most of the measured changes. Furthermore, Fig. 4.9 indicates that there is a significant overlap between changes that occurred in samples with and without recalcification. The changes observed in the dilute blood samples are attributed to a combination of temperature equilibration, $\mathrm{RBC}$ sedimentation and $\mathrm{RBC}$ aggregation. While temperature and aggregation effects generally occur within the first several minutes, sedimentation can be an ongoing process and thus explains why most of the changes occurred gradually throughout the experiment.

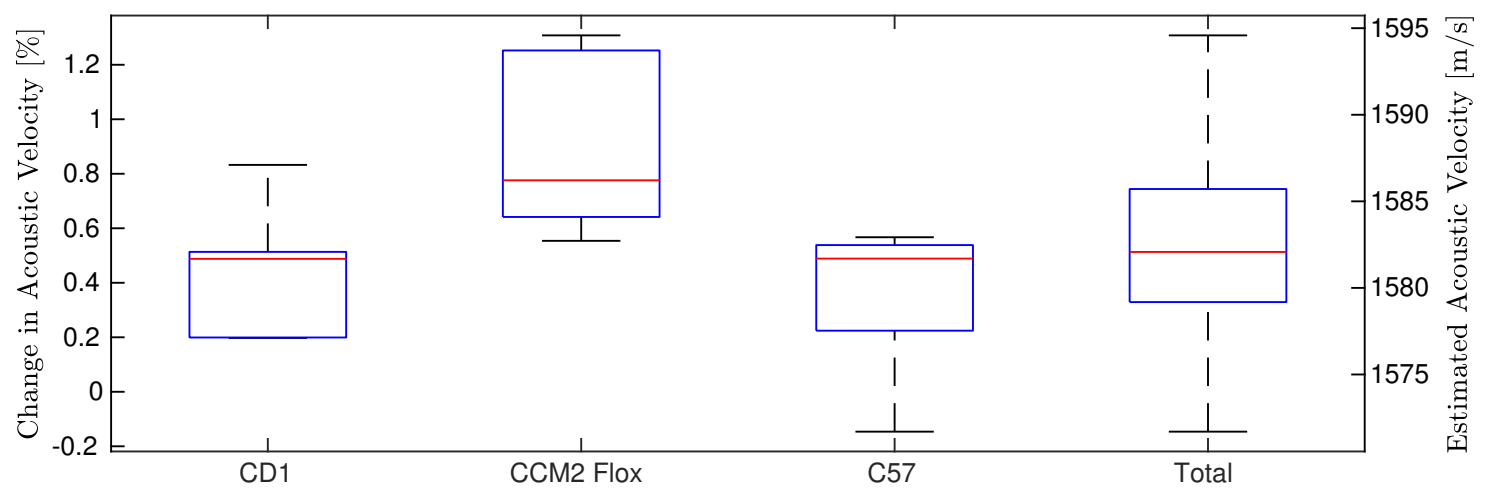

Figure 5.1: Box plots are shown to represent the distribution of acoustic velocity measurements 40 minutes after coagulation. The left axis shows the percentage change in acoustic velocity relative to the initial time point, while the right axis estimates the final acoustic velocity assuming an initial sound speed of $1574 \mathrm{~m} / \mathrm{s}$. 


\subsubsection{M-mode}

\section{Identifying coagulation}

M-mode Imaging allows for the detection of changes in a particular A-Mode trace. This provides a convenient visualization of the motion of structures and scatterers along the scan-line of interest. The extent of correlation between adjacent time points in the image can act as an indicator of how quickly the scatters are moving into or out of the frame. When the scatterers are fixed within a medium, very little change between adjacent time points should be observed. These effects are seen by observing the samples presented in Fig. 4.10. Fig. 4.10(a) shows a sample of blood in a liquid state, which has been diluted with buffer to a concentration of $66 \%$. In this image, there was very little correlation between subsequent A-lines acquired throughout the experiments. This can be seen in the substantial signal fluctuations along horizontal lines. The reason for this lack of correlation can largely be attributed to the sample's dilution. With decreasing RBC concentration, scatterers are able to move more freely in the sample. Furthermore, previous research has concluded that the dilution of blood results in decreased RBC aggregation [63]. A particle's mobility is inversely proportional to its radius [83]. In other words, the decreased aggregation caused by the sample dilution ultimately resulted in increased scatterer motion. Focusing on the bottom right corner of the image, an apparent increase in the correlation from one time point to the next can be observed. As the ROI was selected in close proximity to the surface of the well (which was generally located at an arrival time of $6 \mu \mathrm{s}$ ), the increased correlation corresponded to sedimentation (or settling) of the RBC at the bottom of the sample. There is an apparent build up of sedimentation observed throughout the 30 minute acquisition.

Fig. 4.10(b) again shows a sample of blood in a liquid state. However, in this case the sample was minimally diluted, with $90 \%$ of the sample volume composed of citrated whole blood. Relative to the $66 \%$ blood sample, the M-mode image appears to have a high degree of correlation. This is caused by the increased concentration of whole blood which has the dual effect of decreased freedom of motion and increased RBC aggregation. It is possible that the fluctuations observed in the first 3-4 minutes of acquisition indicate that aggregates were not yet fully formed. It is also interesting to note the downward trend of the scatterers with time. Although sedimentation is not visible at the bottom of the frame, the downward drift of the scatters indicate that sedimentation was occurring nonetheless.

The sample in Fig. 4.10(c) has been recalcified, thereby initiating the coagulation cascade. The clot formation itself resulted in an immobilization of the scatterers (which can be RBCs or RBC aggregates). This can be seen in the M-mode image approximately 14 minutes into the acquisition, where the lack of motion caused a cessation in backscatter fluctuation at a 
certain arrival time. The small variations observed between 14 and 30 minutes were likely a result of ongoing reorganizations that occurred within the clot's microstructure. Prior to the 14 minute mark, a lack of correlation was observed despite the fact that the image shows a highly concentrated blood sample. It is hypothesiszed that the reason the M-mode image does not exhibit similar trends to those seen in Fig. 4.10(b) is that the blood is not truly in a liquid state. In other words, the early stages of blood coagulation had already begun. Although the scatterers had not yet become trapped in the network of fibrin polymers, the formation of these networks was responsible for the motion of the scatterers across the A-Mode frame faster than they would have drifted in a non-coagulating blood sample.

\section{Variability in Onset Time}

Given that M-mode imaging can be used to monitor the onset of clot formation, it has been applied to investigate the variability observed throughout the data collected. Intrasample variability is of particular interest as previous researchers have operated under the assumption that the medium is homogeneous. Fig. 4.11 shows three M-mode images all acquired from the same sample, but at locations that were $420 \mu \mathrm{m}$ apart. Dashed lines have been inserted as an estimate of clot formation time. The onset at each location was relatively consistent along the axial direction, showing that there was not a significant depth dependence of the onset time. However, from left to right the onset times shown were estimated to be 14.5 minutes, 11 minutes and 8.5 minutes. Thus, the onset time varied by 6

minutes across a distance of less than $1 \mathrm{~mm}$. Different samples exhibited this effect to varying degrees, with the largest variation in onset time of 20.5 minutes over a lateral distance of $1.96 \mathrm{~mm}$, and the smallest of 1 minute over a lateral distance $1.82 \mathrm{~mm}$. The distribution of onset times across all locations of all recalcified samples are summarized in Fig. 5.2. The range of onset times are shown for 15 of the 16 samples across all mouse strains. One of the C57 samples has been removed, as clot retraction interfered with the assessment of onset time. 


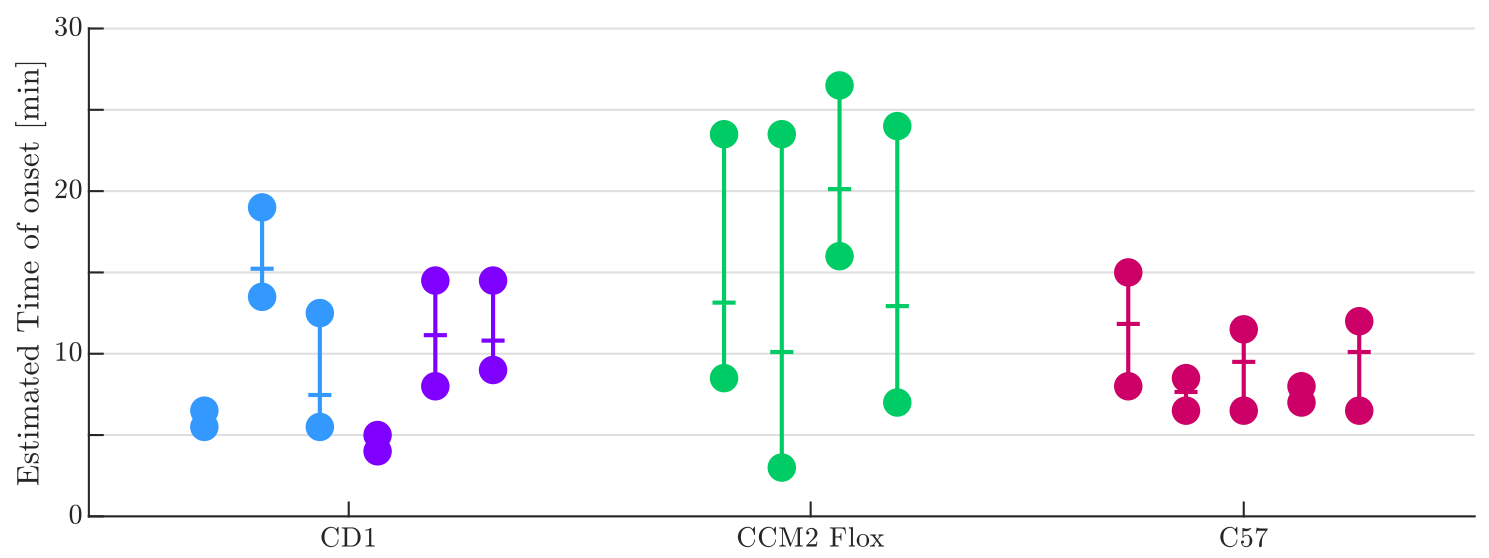

Figure 5.2: The range in onset times is shown for each recalcified sample of each mouse strain, with the mean onset time indicated by a horizontal bar. The CD1 data was acquired on two separate days, denoted by different colours.

Firstly, it should be noted that the CD1 data was collected on two separate days with the first three on day 1 and the second three on day 2. For CCM2 Flox, as for C57, all experiments for the given strain were acquired in a single day and the samples are displayed in the order in which they were acquired.

Each mouse strain has unique genetic features designed for specific research purposes. Although no literature has reported on irregularities in the hemostatic system of any of the strains used, it is possible that differences exist. This could explain the large variability of onset times seen in the CCM2 Flox strain, and the reason that the three strains do not exhibit similar trends. Differences observed within each strain may occur for a number of reasons. The blood used in sequential experiments were stored for different amounts of time, which may have impacted the coagulation kinetics. Additionally, the specific samples used in each case could have had minor variations in the concentrations of blood components. Larger data sets would be necessary to properly assess the cause of variability. However, intra-individual variability has been previously reported and is likely an unavoidable result of minor biological differences [55].

Accounting for all onset times of all strains, the mean and standard deviation of onset times can be calculated as $10.6 \pm 5.0$ minutes. This agrees with the onset times of $11.15 \pm 7$ minutes listed by Callé et al. [16] for rat blood. It should be noted that the values published Callé et al. [16] represent only the variability observed across separate samples, while the value reported here also accounts for intra-sample variability in onset time. 


\section{Structural Variability}

In addition to the variability of onset time, discrepancies in M-mode signals provide insight into the structural differences both across different samples and within a given sample. Four representative M-mode images are shown in Fig. 4.12 in an attempt to highlight these differences.

In Fig. 4.12(a), clot retraction from the well's surface was observed, identified by the lack of backscatter at the bottom of the frame. This effect has previously been shown in a B-Mode image seen in Fig. 4.6. In this case, the onset of formation was still visible at $\sim 3$ minutes. However, in some cases (as was previously mentioned about one of the C57 samples), the onset was obscured by the retraction and did not allow proper assessment. As will be discussed in the following section, retraction can also impact frequency data and without M-mode for verification could be mistaken as the onset time. Even in cases where the retraction does not occur along the A-Mode trace of interest, the data can still be affected. This can be observed in Fig. 4.12(b) in the form of a shift at $\sim 22$ minutes. Again, this can impact the reflection spectra and make it difficult to interpret without the complimentary M-mode image. In the cases where retraction occurred, it was generally observed at all locations within the sample, either with visible retraction as seen in Fig. 4.12(a), or shifts due to out-of-frame retraction as in Fig. 4.12(b).

Fig. 4.12(c) shows a coagulating sample exhibiting pockets in the central region of the clot that do not exhibit any backscatter, and the formation of areas with very high backscatter (bright yellow lines). Given that clot retraction typically involves the clot pulling in on itself, the appearance of these internal pockets with an apparent lack of US scatterers might not be expected. The high backscatter (bright yellow lines) may be related to the aggregation of RBCs in specific regions of the clot, highlighting the heterogeneous nature of the process. This acts to highlight the degree of complexity observed within these samples. Just as these regions exhibit limited axial spans, the same was observed laterally. In other words, in two adjacent scan-lines (with a separation of $140 \mu \mathrm{m}$ ), one may exhibit regions lacking backscatter while the other may show a conventional clot structure (e.g. that shown in Fig. 4.11). As with the clot retraction, these regions impact the nature of the frequency data. This effect will be further discussed in the subsequent section.

Finally, Fig. 4.12(d) is included to show that occasionally it is difficult to assess whether or not complete formation as occurred regions within the clot. In this case, fluctuations in the signal intensity are observed axially between 6.2 and $6.5 \mu \mathrm{s}$. It is unclear whether or not this indicated partial or incomplete formation or if it simply corresponded to a less dense region of the fibrin mesh where scatterers had a more limited range of motion. 


\section{Summary}

M-mode imaging has proven to be a useful tool for visualizing changes in the clot microstructure as a function of time. The main limitation of M-mode imaging in the context of this work is the lack of quantitative information. Early attempts were made to utilize metrics that assess correlation between adjacent time points in an attempt to quantitatively identify clot onset. This proved difficult and ultimately ineffective as the metrics were highly sensitive to any motion within the sample. Consequently, the onset time was generally over-estimated. Furthermore, correlation maps remove the utility of the M-mode images in identifying features in the clot such as in-frame and out-of-frame retraction, regions with decreased backscatter, and partial or incomplete formation.

\subsubsection{Reflection spectra}

As has previously been mentioned, the spectra acquired in this work are obtained from the pulse reflected off of the cap's surface. This means that changes in the reflection spectra occur as a result of cumulative changes throughout the region along which the pulse travels. All spectra are normalized such that each frequency component has a maximum intensity of $0 \mathrm{~dB}$, regardless of the time point at which this maximum occurred.

For the sake of comparison to previously published results, it is important to remember that the spectra shown here reflect relative intensity of the signals, while other research commonly reports results in the form of frequency dependant attenuation. As has been discussed in Sec. 3.1.5, the attenuation coefficient is inversely proportional to the signal intensity and has been normalized to some reference spectrum (plane reflector) that has been acquired in the same system. Additionally, the attenuation coefficient is typically expressed in units of $\mathrm{dB} / \mathrm{cm}$ thus incorporating how much the wave is attenuated per unit distance.

It was not possible to calculate the attenuation coefficient due to current limitations of the system that prevent the acquisition of a reliable reference spectrum. Three factors pertaining to the well cap prevent the normalization. Firstly, aligning the transducer with the same cross-section of the plug is virtually impossible. Each experiment will thus cover a slightly different span of the plug, with some closer to the centre of the plug's radius than others. This is the reason that the number of locations analyzed for each sample ranges from ten to fourteen. This, combined with the uneven surface of the cap, means that reflected pulses will have slightly different spectra depending where the pulse is reflected from. If one were to normalize a spectrum acquired in blood to one acquired in water, it is possible that differences between the two spectra are simply a result of different interactions between the pulse and the 
cap's surface rather than difference between the wave propagation in the medium. Finally, the variability of the plug's location axially, combined with unknown acoustic velocities in the sample medium means that the distance the wave travels is unknown. The distance travelled will affect the intensity of the reflected wave, which will in turn affect the calculated attenuation coefficient, since in this system the distance cannot be properly accounted for. Furthermore, the normalization would be inaccurate due to different distances travelled between the sample measurement and reference measurement.

In future iterations of the system, it may be possible to eliminate some of the discrepancies associated with the plug in order to calculate a reasonable estimate for the attenuation coefficient. This would involve redesigning the well cap with a smooth surface relatively to the US wavelength. In water, the wavelength at $80 \mathrm{MHz}$ is roughly $19 \mu \mathrm{m}$. Generally speaking a surface can be considered smooth for if it has variations less than $1 / 10^{\text {th }}$ the wavelength, which would correspond to roughly $2 \mu \mathrm{m}$ in this case. The difficulty in obtaining a custom designed cap with this degree of precision is the reason that it wasn't achieved for this work. Furthermore, the distance between the bottom of the well and the well-cap would need to be fixed and accurately measured.

With these limitations in mind, the signal intensity can be thought of as inversely proportional to the attenuation. An increase in the signal intensity means that the wave is less attenuated and corresponds to what would be a decrease in the attenuation coefficient of the medium.

The figures included in Sec. 4.2.3 aim to highlight how the spectrum changes as a result of changes in the sample's microstructure. Each spectrum corresponds to one of the M-mode figures included in Sec. 4.2.2. The discussion will be broken up into the same sections as those in the previous section.

\section{Identifying coagulation}

Fig. 4.13 shows three samples, two of which are dilutions are citrated blood with the third presenting a recalcified sample. The M-mode image depicting the kinetics of these samples can be seen in Fig. 4.10. In the 66\% WB solution in Fig. 4.13(a), the spectral trends can be divided into two regions. From $25-65 \mathrm{MHz}$, a slow decrease in the signal intensity of 1-1.5 $\mathrm{dB}$ is observed, which could be a result of temperature change or sedimentation. From 65-95 $\mathrm{MHz}$, fluctuations of 5-10 dB are observed for the duration of the experiments. It is thought that these trends are caused by the small scatters (individual RBC or small aggregates) moving quickly in and out of the frame.

In Fig. 4.13(b), the spectrum exhibits fluctuations of 1-2 $\mathrm{dB}$ across all frequencies throughout the experiment. The increased fluctuations in the lower frequency range are 
thought to be caused by the increased scatter sized due to RBC aggregation.

In the recalcified sample shown in Fig. 4.13(c), coagulation is visible by a drop in the signal intensity. This implies that the blood clot is more attenuating than the blood in its liquid state. It is hypothesized that the bright region located between 8 and 14 minutes, corresponds to the transition phase where the blood is undergoing major changes to form the structure of the resulting clot. Aside from perhaps minor differences in the apparent correlation, this feature is not visible in the M-mode image. This region of high intensity is particularly prominent at higher frequencies, and was reproduced in the majority of coagulating samples.

\section{Variability in onset time}

The effects described for Fig. 4.13(c) can be seen for three locations of a different sample in Fig. 4.14. The dashed line corresponds to the predicted onset time based on stabilization of the M-mode signals. Again, in the higher frequency region, an increase in the signal intensity is observed prior to the clot onset and is thought to be a result of changes caused by the initial phases of clot formation. On a biological scale, this could correspond to the polymerization of the fibrin monomers in the early stages of the network formation prior to the immobilization of RBC. It is difficult to compare these trends and the hypothesis being made to the results obtained by other groups due to the different frequency range used. Previous studies have noted significant frequency dependence of trends observed at frequencies below $30 \mathrm{MHz}$. For example, a recent study by Taturanas et al. noted that an increase the in spectral intensity was observed at $10 \mathrm{MHz}$ during the clot formation stage followed by a decrease once the clot was fully formed. At $20 \mathrm{MHz}$, however, they observed a decrease in the intensity during the formation followed by an increase in the intensity once the clot was formed [84]. This draws into question the use of parameters such as the integrated attenuation coefficient, which as the name implies, involves an integration of the attenuation across a range of frequencies. If there are significant differences in the frequency dependant attenuation at the various stages of blood coagulation, valuable information may be lost in the integration process. While certain theories have been proposed to explain how the IAC corresponds to the stages of coagulation, they have not yet been validated in published work. Decreases in the IAC have been observed prior to the clot stabilization $[15,69]$ which may correspond to the same effect observed here by the increased intensity. Following the complete stabilization however, the IAC has been reported to undergo extreme fluctuations and variability and is not deemed a reliable metric.

Regardless, the decrease in signal intensity across all frequencies coincides temporally with the immobilization of scatterers observed in the M-mode images presented in this work. 
This provides a strong argument to the fact that the US wave is more highly attenuated in the coagulated sample that it is prior to the clot's formation.

\section{Structural variability}

As discussed in Sec. 5.2.2, apparent variations in clot microstructure were observed. Examples of inhomogeneities in the clot's microstructure are shown in M-mode images in Fig. 4.12. The reflection spectra corresponding to the reflected pulse along the same A-Mode lines can be seen in Fig. 4.15.

In Fig. 4.15(a), which shows data acquired from a location with clot retraction from the well, the clot onset occurred at $\sim 3$ minutes. The onset can be visualized by an increased intensity in the first several minutes of acquisition followed by a decrease once the clot has formed. Following the retraction, which occurs at roughly 8 minutes into the experiment, an increase in intensity is seen. This is thought to occur due to the large region of the scan-line which no longer contained scatterers. It is hypothesized that the non-scattering region was serum, which is essentially blood plasma with reduced concentrations of certain coagulant proteins. Previous studies have shown that plasma is less attenuating than whole blood [69]. At $80 \mathrm{MHz}$, it is estimated that the attenuation in plasma is approximately $20 \mathrm{~dB} / \mathrm{cm}$, while in blood it is $87 \mathrm{~dB} / \mathrm{cm}$, showing more than a fourfold difference [43]. Under the assumption that the acoustic properties of serum and plasma are comparable, this could explain the increased signal intensity.

For out-of-frame retraction, a shift in the reflection spectrum was observed (Fig. 4.15(b)). The out-of-frame retraction is visible as a decrease in signal intensity approximately 22 minutes into the experiment. While in this case, the out-of-frame retraction caused a decrease in signal intensity, in other locations and samples it has been shown to increase instead. It is likely that the shift in the reflection spectra is a result of a slightly different conformation of scatterers and of minor differences in the microstructure between the original and the shifted region of the clot. Thus, the changes in out-of-frame retraction signal intensity occurred for different reasons than those discussed when the retraction occurred along the A-Mode trace of interest.

An example of the uncommon situation where regions with limited backscatter appeared within the clot rather than at the edge of the vessel is shown in Fig. 4.15. Compared to the usual decrease in signal intensity across all frequencies, an increase in the intensity was observed at higher frequencies $(\sim 75--95 \mathrm{MHz})$. The frequency trends were relatively consistent for all samples exhibiting this effect. Assuming the region with limited backscatter was filled with serum, it is possible that the increase in signal intensity was caused by the decreased attenuation in these regions. A counterargument is that it is unclear why this 
effect is not observed across all frequencies.

Finally, the reflection spectra corresponding to the M-mode image with visible fluctuations in the formed clot region is shown in Fig. 4.15(d). In general, the observed trends were similar to what has previously been shown for typical coagulation (Fig. 4.14 and Fig. 4.13(c)). However, the transition from the high to low intensity regions is more gradual in this case, particularly at the lower frequencies. It is thought that this gradual transition is caused by either incomplete formation or less rigid formation than is conventionally seen.

\section{Summary}

Features observed in the frequency domain have been shown to correlate well with effects shown in the M-mode images presented in Sec. 4.2.2 and discussed in Sec. 5.2.2. It is possible that additional insight into the kinetics prior to clot stabilization may be gained using frequency data in conjunction with M-mode images. It is thought that the increased intensity observed prior to formation may be an indication of early stages of blood coagulation. Given the tendency for these trends to become more pronounced at higher frequencies, this HFUS system is well suited for further investigation into these effects.

\subsubsection{Comparisons}

In this section, comparisons between trends observed in acoustic velocity, M-mode images, and reflection spectra are discussed. While some of the talking points have been covered in previous sections, they are reiterated here to highlight how certain features compare between the different modalities.

Starting with Fig. 4.16, we observe the summarized data for two dilute blood samples, and one recalcified sample. The specific trends observed in the frequency domain and Mmode images of the dilute blood samples (Fig. 4.16(a) and (b)) have already been discussed. The fluctuations in the reflection spectra are made clear in the line plot overlayed onto the M-mode image. The acoustic velocity measurements for these particular samples showed a gradual increase over time. In Fig. 4.16(c), the line plot corresponding to the intensity of the $80 \mathrm{MHz}$ signal was able to clearly highlight the agreement between the features that occurred in the frequency data and those seen in the M-mode image. Furthermore, the acoustic velocity seemed to show similar kinetics, with a sharp increase occurring around the same time as the clot underwent formation. However, as shown in Fig. 4.17 and highlighted below, this correlations was not observed for all locations.

In Fig. 4.17, three locations from a single sample of recalcified whole blood are shown. The kinetics between the reflection spectra and the M-mode are emphasized by the overlayed line 
plot. Here, lack of sensitivity of the velocity is highlighted. Despite the significant changes in the time of onset across the three locations, the velocity remained virtually identical.

Regarding clot retraction, shown in Fig. 4.18(a), we see that the frequency trace at $80 \mathrm{MHz}$ was less suitable for the identification of important features than was the full spectrum. There was a drop of over $12 \mathrm{~dB}$ at the point of formation ( $\sim 3$ minutes) in the $80 \mathrm{MHz}$ data; however, the fact that the dip immediately recovered made it difficult to separate from the other signal fluctuations. Furthermore, the arc that appeared between 14 and 27 minutes following the start of acquisition was significant and obscured some of the other trends. In this case, it was more helpful to compare the full spectrum to the M-mode, upon which the trends discussed in Sec. 5.2.3 still apply. The velocity appeared to increase with the onset of clot formation and was unaffected by the retraction from the measurement cell.

In the out-of-frame retraction (Fig. 4.18(b)), the frequency trace at $80 \mathrm{MHz}$ can be difficult to interpret on its own due to additional fluctuations that occurred around the main features. As previously discussed, an increase still occured prior to clot formation (between 5 and 7 minutes), followed by a drop of almost $12 \mathrm{~dB}$ at the time of onset. A drop of $4 \mathrm{~dB}$ was also observed at the time of out-of-frame retraction (22 minutes). The acoustic velocity dropped before undergoing a gradual increase following the onset of clot formation.

When regions of decreased backscatter occurred within the clot, the kinetics between Mmode and frequency were still apparent (Fig. 4.19(a)). The line plot shows a $\sim 6 \mathrm{~dB}$ increase following clot onset. Once the clot had formed, the fluctuations at $80 \mathrm{MHz}$ were minor. In this case the changes in acoustic velocity ceased with the onset of clot formation.

Finally, partial or incomplete clot formation resulted in a gradually declining intensity in the reflection spectra. Fig. 4.19 shows this spectral trend overlayed onto the corresponding M-mode image. It is possible that the decrease of $\sim 6 \mathrm{~dB}$ between 4.5 and 5.5 minutes corresponded to the point where most of the clot had formed. The acoustic velocity in this figure did not appear to correlate with any of the features observed in either the reflected spectrum or the M-mode image. 


\section{Chapter 6}

\section{Summary and Conclusions}

The work performed for this thesis investigated the use of HFUS as a global coagulation test for whole blood samples. System modifications were performed in order to achieve a setup similar to that used in the OHP, which relies on optical transmittance through small volume samples of plasma to assess the coagulant and fibrinolytic cascades. Ultrasound, which can propagate through optically opaque materials such as whole blood, was then used to monitor acoustic changes in both plasma and whole blood during coagulation.

Experiments performed using blood plasma provided supportive evidence for previous reports that the acoustic properties in plasma do not change significantly during coagulation. Moreover, it was found that there were no significant changes in the acoustic properties as a result of clot breakdown. Proof-of-principle experiments using murine whole blood highlighted the technique's ability to detect kinetic changes in the sample during coagulation. M-Mode images were sensitive to clot formation time and provided a means of visualizing irregularities in the clot's structure. Furthermore, spatial information acquired by scanning the transducer across the well provided insight into previously unreported coagulation onset time variations. Frequency spectra were also shown to be sensitive to the onset time; however, in certain instances such as during in-frame clot retraction, spectral features could be difficult to interpret without the aid of the M-Mode image. A feature of particular interest was an increase in the spectral intensity (or a decrease in the US attenuation) at higher frequencies observed in the minutes prior to the clot onset. It is hypothesized that this corresponds to the early stages of clot formation while fibrin polymerization is underway but RBC have not yet been immobilized. This indicates the spectral data are sensitive to changes that aren't visible using the M-Mode data alone.

Acoustic velocity measurements were found to be highly variable between samples and did not correlate well with the kinetics observed in M-Mode images or the frequency spectrum. This contradicted reports that the acoustic velocity can be used as an indicator of clot formation. Additional experiments are required to determine whether or not this can be attributed to differences between murine and human samples.

Moving forward, this system will be used to perform measurements of human blood. In these experiments, a complete hemostatic assessment could be performed by introducing a fibrinolytic agent to induce clot breakdown. While these experiments were not possible 
in murine blood due to differences in their hemostatic pathways (namely, their strong resistance to fibrinolysis), it is expected that the measurement techniques presented here for the assessment of clot formation will apply equally well to clot breakdown. Further system refinements pertaining to the well cap could enable the calculation of absolute rather than relative values as were presented in this work. Absolute measurements of acoustic velocity or attenuation would provide added value to the quantitative assessment of measured ultrasonic parameters. Additionally, improving the temporal resolution with which data can be acquired is desirable, and would make it feasible to monitor fast processes such as clot formation following activation using thrombin.

The SASAM system is well equipped for photoacoustic analysis. In addition to the US results shown here, PA could be used to gain added insight into the coagulating blood sample, such as blood oxygenation levels, or overall HCT levels. 


\section{Appendix A}

\section{Dilution Protocols}

As with all biochemical experiments, the ability to appropriately alter stock concentrations is crucial. The well known formalism relating a dilute solution to the stock solution can expressed as:

$$
C_{1} V_{1}=C_{2} V_{2},
$$

where, $C_{1}$ and $V_{1}$ represent the concentrations and volumes of the stock solution, while $C_{2}$ and $V_{2}$ are the concentrations and volume of the final dilutions. Typically, the stock concentration and volume are known as is the desired concentration of the dilution. The equation can be rearranged to solve for the total volume of the dilute sample. Alternatively, the desired volume of the dilute sample may be known, in which case one could solve for the required volume of the stock solution.

\section{A.1 Buffer Dilutions}

Three buffers were used for the hematological assays, and their uses are described in Sec. 3.3.1. The solutions were typically prepared in $50 \mathrm{~mL}$ centrifuge tubes, and the relative volumes of each constituent can be found in Tab. A.1. After preparing a given buffer, the centrifuge tubes should be sealed and vortexed to ensure adequate mixing. The solution then passed through a $200 \mathrm{~nm}$ Polyethersulfone (PES) membrane syringe filter. 


\begin{tabular}{|c|c|c|c|}
\hline Ingredients & Initial Concentration & Final Concentration & Volume \\
\hline \multicolumn{4}{|c|}{ Plate Blocking Solution $(50 \mathrm{~mL})$} \\
\hline $\mathrm{H}_{2} \mathrm{O}$ & - & - & $40 \mathrm{~mL}$ \\
\hline $\mathrm{HBS}$ & $5 \mathrm{X}$ & $1 \mathrm{X}$ & $10 \mathrm{~mL}$ \\
\hline Tween $\AA 20$ & $100 \%$ & $1 \%$ & $500 \mu \mathrm{L}$ \\
\hline \multicolumn{3}{|c|}{ Assay Buffer $(50 \mathrm{~mL})$} \\
\hline $\mathrm{H}_{2} \mathrm{O}$ & - & - & $40 \mathrm{~mL}$ \\
\hline $\mathrm{HBS}$ & $5 \mathrm{X}$ & $1 \mathrm{X}$ & $10 \mathrm{~mL}$ \\
\hline Tween $\AA 20$ & $100 \%$ & $0.01 \%$ & $5 \mu \mathrm{L}$ \\
\hline \multicolumn{4}{|c|}{} \\
\hline $\mathrm{H}_{2} \mathrm{O}$ & Assay Buffer with Prionex $(50 \mathrm{~mL})$ & \\
\hline $\mathrm{HBS}$ & - & - & $40 \mathrm{~mL}$ \\
\hline Tween $\AA 20$ & $5 \mathrm{X}$ & $1 \mathrm{X}$ & $10 \mathrm{~mL}$ \\
\hline Prionex & $100 \%$ & $0.01 \%$ & $5 \mu \mathrm{L}$ \\
\hline
\end{tabular}

Table A.1: Dilution protocols for buffers used in hematological assays.

\section{A.2 Clotting Factor and Fibrinolytic Enzyme Dilutions}

Three constituents are added to the assay to initiate the coagulant and fibrinolytic cascades. Thrombin (clotting factor IIa) and calcium (clotting factor IV) play important roles in clot formation, while urokinase plasminogen activator $(\mathrm{uPa})$ initiates clot breakdown. All three are acquired in concentrated stocks that must be appropriately diluted to achieve the desired kinetics.

The preliminary dilutions of IIa and uPa are discussed in Sec. 3.3.2. The concentrations of the these preliminary dilutions can vary between bulk orders, therefore specific dilution volumes must be calculated using Eq. A.1 on a case to case basis. Generally they were prepared on the day of the experiment into $300 \mu \mathrm{L}$ volumes. The desired concentration of the $300 \mu \mathrm{L}$ solution, and the resultant working concentration of the enzyme when a droplet of the $300 \mu \mathrm{L}$ solution is diluted by other sample components to a total volume of $100 \mu \mathrm{L}$ in the well are shown in Tab. A.2. 


\begin{tabular}{|c|c|c|c|}
\hline Buffer & 300 $\mu$ L Stock Concentration & Volume in Well & Working Concentration \\
\hline \multicolumn{4}{|c|}{ Thrombin } \\
\hline Prionex & $100 \mathrm{nM}$ & $5 \mu \mathrm{L}$ & $5 \mathrm{nM}$ \\
\hline \multicolumn{4}{|c|}{ Urokinase Plasminogen Activator } \\
\hline Prionex & $400 \mathrm{nM}$ & $5 \mu \mathrm{L}$ & $20 \mathrm{nM}$ \\
\hline \multicolumn{4}{|c|}{ Calcium Chloride } \\
\hline Prionex & $750 \mathrm{mM}$ & $2 \mu \mathrm{L}$ & $15 \mathrm{mM}$ \\
\hline
\end{tabular}

Table A.2: Enzyme concentrations used for initiation of the coagulant and fibrinolytic pathways. 


\section{Appendix B}

\section{System Refinement Experiments}

\section{B.1 Meniscus}

A colour map representing signal intensity at the clot-air interface can be seen in Fig. B.1(a). Based on the correlation between signal intensity and the depth of water, which is shown in Fig. B.1(b), the variability is attributed to the curvature of the meniscus. Water depth was calculated using time of flight and assuming a sound speed of $1524 \mathrm{~m} / \mathrm{s}$ for water at $37^{\circ}$. It could not be determined where there was no visible reflection from the surface, so dark blue in the majority of the well corresponds to immeasurable values. The white ring represents the approximate location of the inner radius of the well.

a)

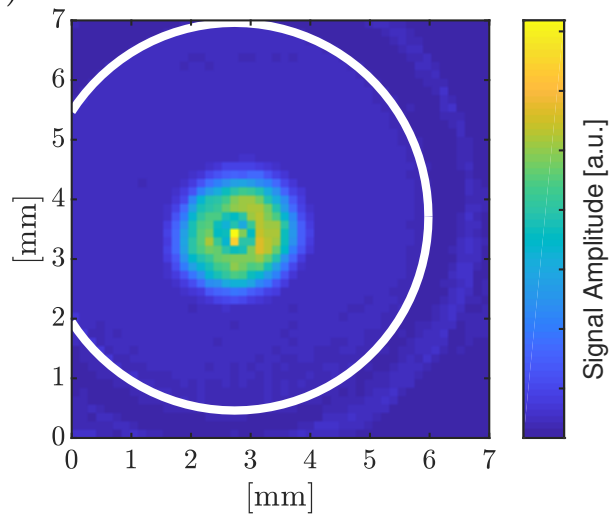

b)

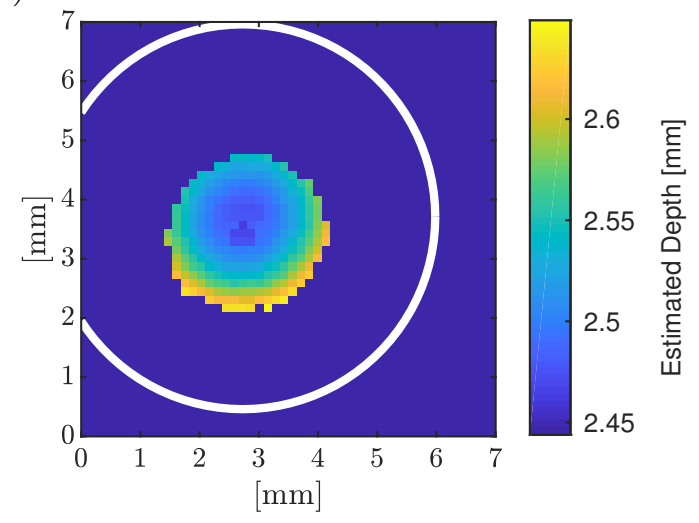

Figure B.1: a) The signal amplitude at the surface of a water sample is shown as a function of spatial location in a $50 \times 50$ scan. b) The estimated depth of the water (i.e. the height of the surface) is shown for the same scan. In both images, the white circle estimates the inner radius of the well. 


\section{B.2 Acquisition Automation}

The data acquisition script used to automatically collect data every 30 seconds can be seen in Program B.1

$\overline{\text { Program B.1 Autoit script that collects and saves a } 50 \text { point scan line every } 30 \text { seconds }}$ for 40 minutes.

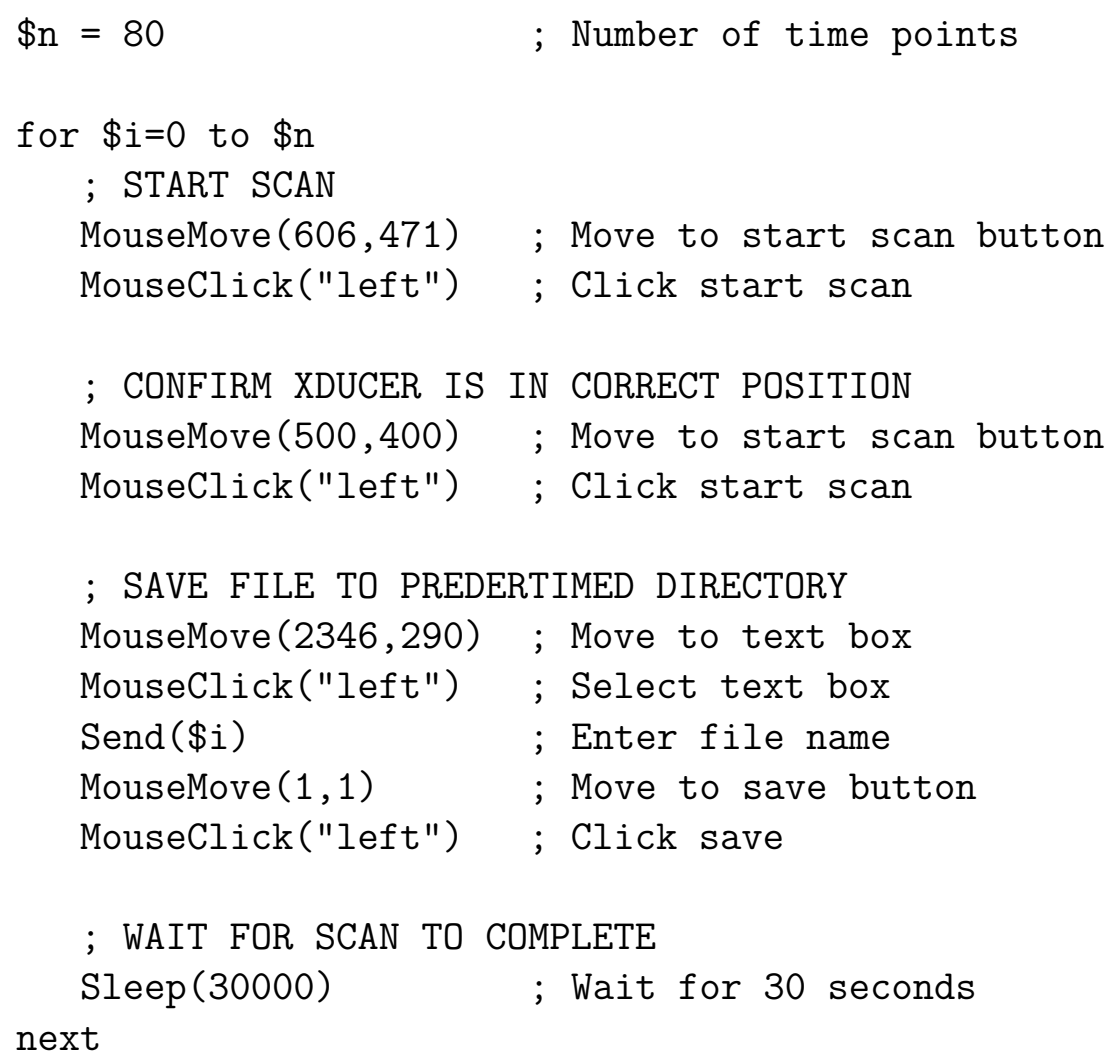




\section{Bibliography}

[1] RA Adams, C Schachtrup, D Davalos, I Tsigelny, and K Akassoglou. Fibrinogen signal transduction as a mediator and therapeutic target in inflammation: lessons from multiple sclerosis. Current medicinal chemistry, 14(27):2925-2936, 2007.

[2] Aleksandra Antovic. The overall hemostasis potential: a laboratory tool for the investigation of global hemostasis. In Seminars in thrombosis and hemostasis, volume 36, pages 772-779. (C) Thieme Medical Publishers, 2010.

[3] AE Aubert, H Kesteloot, H De Geest, and W Van Dael. Measurement of high frequency sound velocity in blood. Biosigma, v(1):421-425, 1978.

[4] S Paul Bajaj and J Heinrich Joist. New insights into how blood clots: implications for the use of aptt and pt as coagulation screening tests and in monitoring of anticoagulant therapy. In Seminars in thrombosis and hemostasis, volume 25, pages 407-418. Copyright(c) 1999 by Thieme Medical Publishers, Inc., 1999.

[5] T Bakke, T Gytre, A Haagensen, and L Giezendanner. Ultrasonic measurement of sound-velocity in whole-blood - comparison between an ultrasonic method and conventional packed-cell-volume test for hemtocrit determination. Scanidinavian Journal of Clinical \& Laboratory Investigation, 35(5):473-478, 1975.

[6] M Besser, C Baglin, R Luddington, A van Hylckama Vlieg, and T Baglin. High rate of unprovoked recurrent venous thrombosis is associated with high thrombin-generating potential in a prospective cohort study. Journal of Thrombosis and Haemostasis, 6(10):1720-1725, 2008.

[7] Annabel Blasi, Andrea Calvo, Verónica Prado, Enric Reverter, Juan Carlos Reverter, María Hernández-Tejero, Fátima Aziz, Alex Amoros, Andres Cardenas, and Javier Fernández. Coagulation failure in patients with acute-on-chronic liver failure and decompensated cirrhosis: Beyond the international normalized ratio. Hepatology, 68(6):23252337, 2018. 
[8] Blausen Staff. Medical gallery of blausen medical 2014. Wikijournal of Medicine, 1(2), 2014. Accessed 08/08/2019.

[9] PG Board, MS Lososky, and KJA Miloszewski. Factor xiii: inherited and acquired deficiency. Blood reviews, 7(4):229-242, 1993.

[10] Daniel Bolliger, Manfred D Seeberger, and Kenichi A Tanaka. Principles and practice of thromboelastography in clinical coagulation management and transfusion practice. Transfusion medicine reviews, 26(1):1-13, 2012.

[11] Julian I Borissoff, Ivo A Joosen, Mathijs O Versteylen, Henri M Spronk, Hugo ten Cate, and Leonard Hofstra. Accelerated in vivo thrombin formation independently predicts the presence and severity of ct angiographic coronary atherosclerosis. JACC: Cardiovascular Imaging, 5(12):1201-1210, 2012.

[12] E.L. Bradley and Jose Sacerio. The velocity of ultrasound in human blood under varying physiologic parameters. Journal of Surgical Research, 12(4):290 - 297, 1972.

[13] Marisa A Brake, Lacramioara Ivanciu, Susan A Maroney, Nicolas D Martinez, Alan E Mast, and Randal J Westrick. Assessing blood clotting and coagulation factors in mice. Current protocols in mouse biology, 9(2):e61, 2019.

[14] E Brown, B Bradley, R Wennesland, JL Hodges, J Hopper, and H Yamauchi. Red Cell, Plasma, and Blood Volume in Healthy Women Measured by Radichromium CellLabeling and Hemotocrit. Journal of Clinical Investigation, 41(12):2182-2190, 1962.

[15] Rachel Calle, Camille Plag, Frederic Patat, and Frederic Ossant. Interest of the attenuation coefficient in multiparametric high frequency ultrasound investigation of whole blood coagulation process. Journal of the Acoustical Society of America, 125(1):530-538, JAN 2009.

[16] Rachel Callé, Gaël Y Rochefort, Nicolas Desbuards, Camille Plag, Daniel Antier, and Frédéric Ossant. Evaluation of the sensitivity of an in vitro high frequency ultrasound device to monitor the coagulation process: study of the effects of heparin treatment in a murine model. Ultrasound in medicine \&3 biology, 36(2):295-305, 2010.

[17] Laure Carcaillon, Martine Alhenc-Gelas, Yannick Bejot, Christian Spaft, Pierre Ducimetière, Karen Ritchie, Jean-François Dartigues, and Pierre-Yves Scarabin. Increased thrombin generation is associated with acute ischemic stroke but not with coronary heart disease in the elderly: the three-city cohort study. Arteriosclerosis, thrombosis, and vascular biology, 31(6):1445-1451, 2011. 
[18] R.S.C. Cobbold. Foundations of Biomedical Ultrasound. Biomedical Engineering Series. Oxford University Press, 2007.

[19] AF Collings and N Bajenov. Temperature dependence of the velocity of sound in human blood and blood components. Australasian physical \& engineering sciences in medicine, 10(3):123, 1987.

[20] Bruce M Coull, Nancy Beamer, Patricia De Garmo, Gary Sexton, Frank Nordt, Robert Knox, and GV Seaman. Chronic blood hyperviscosity in subjects with acute stroke, transient ischemic attack, and risk factors for stroke. Stroke, 22(2):162-168, 1991.

[21] JL Curnow, M-C Morel-Kopp, C Roddie, M Aboud, and CM Ward. Reduced fibrinolysis and increased fibrin generation can be detected in hypercoagulable patients using the overall hemostatic potential assay. Journal of Thrombosis and Haemostasis, 5(3):528534, 2007.

[22] Y Dargaud, AS Wolberg, E Gray, C Negrier, and HC Hemker. Proposal for standardized preanalytical and analytical conditions for measuring thrombin generation in hemophilia: communication from the ssc of the isth. Journal of thrombosis and haemostasis: JTH, 15(8):1704, 2017.

[23] Yesim Dargaud, M Christine Trzeciak, Jean Claude Bordet, Jacques Ninet, and Claude Negrier. Use of calibrated automated thrombinography \pm thrombomodulin to recognise the prothrombotic phenotype. Thrombosis and haemostasis, 96(11):562-567, 2006.

[24] Dimitrios Davalos and Katerina Akassoglou. Fibrinogen as a key regulator of inflammation in disease. In Seminars in immunopathology, volume 34, pages 43-62. Springer, 2012.

[25] Anja Drebes, Marie de Vos, Sunita Gill, Emma Fosbury, Sue Mallett, Andy Burroughs, Banwari Agarwal, David Patch, and Pratima Chowdary. Prothrombin complex concentrates for coagulopathy in liver disease: Single-center, clinical experience in 105 patients. Hepatology communications, 3(4):513-524, 2019.

[26] Vivian X Du, Dana Huskens, Coen Maas, Raed Al Dieri, Philip G de Groot, and Bas de Laat. New insights into the role of erythrocytes in thrombus formation. In Seminars in thrombosis and hemostasis, volume 40, pages 072-080. Thieme Medical Publishers, 2014. 
[27] Juan C Duchesne, Tareq M Islam, Lance Stuke, Jeremy R Timmer, James M Barbeau, Alan B Marr, John P Hunt, Jeffrey D Dellavolpe, Georgia Wahl, Patrick Greiffenstein, et al. Hemostatic resuscitation during surgery improves survival in patients with traumatic-induced coagulopathy. Journal of Trauma and Acute Care Surgery, 67(1):3339, 2009.

[28] Francis A. Duck. Physical properties of tissue: a comprehensive reference book. Academic Press, 1990.

[29] R. Erbel, V. Aboyans, C. Boileau, E. Bossone, R. Di Bartolomeo, H. Eggebrecht, A. Evangelista, V. Falk, H. Frank, O. Gaemperli, M. Grabenwöger, A. Haverich, B. Iung, A.J. Manolis, F. Meijboom, C.A. Nienaber, M. Roffi, H. Rousseau, U. Sechtem, P.A. Sirnes, R.S. Von Allmen, C.J.M. Vrints, J.L. Zamorano, S. Achenbach, H. Baumgartner, J.J. Bax, H. Bueno, V. Dean, C. Deaton, Ç. Erol, R. Fagard, R. Ferrari, D. Hasdai, A. Hoes, P. Kirchhof, J. Knuuti, P. Kolh, P. Lancellotti, A. Linhart, P. Nihoyannopoulos, M.F. Piepoli, P. Ponikowski, J.L. Tamargo, M. Tendera, A. Torbicki, W. Wijns, S. Windecker, M. Czerny, J. Deanfield, C. Di Mario, M. Pepi, M.J.S. Taboada, M.R. Van Sambeek, and C. Vlachopoulos. 2014 esc guidelines on the diagnosis and treatment of aortic diseases. European Heart Journal, 35(41):2873-2926, 2014.

[30] E Ernst, A Matrai, and M Marshall. Blood rheology in patients with transient ischemic attacks. Stroke, 19(5):634-636, 1988.

[31] Jean-Michel Escoffre and Ayache Bouakaz. Therapeutic ultrasound, volume 880. Springer, 2015.

[32] Matthew J Flick, Christine M LaJeunesse, Kathryn E Talmage, David P Witte, Joseph S Palumbo, Malinda D Pinkerton, Sherry Thornton, and Jay L Degen. Fibrin(ogen) exacerbates inflammatory joint disease through a mechanism linked to the integrin $\alpha \mathrm{m}$ $\beta 2$ binding motif. The Journal of clinical investigation, 117(11):3224-3235, 2007.

[33] N Galiè, MM Hoeper, M Humbert, A Torbicki, JL Vachiery, JA Barbera, M Beghetti, P Corris, S Gaine, JS Gibbs, et al. Esc/ers guidelines guidelines for the diagnosis and treatment of pulmonary hypertension. European Respiratory Journal, 34(6):1219, 2009.

[34] Kathryn C Gersh, Chandrasekaran Nagaswami, and John W Weisel. Fibrin network structure and clot mechanical properties are altered by incorporation of erythrocytes. Thrombosis and haemostasis, 102(12):1169-1175, 2009. 
[35] Samuel Z Goldhaber and Henri Bounameaux. Pulmonary embolism and deep vein thrombosis. The Lancet, 379(9828):1835-1846, 2012.

[36] Hiroyuki Hachiya, Shigeo Ohtsuki, Motonao Tanaka, and Floyd Dunn. Determination of sound speed in biological tissues based on frequency analysis of pulse response. The Journal of the Acoustical Society of America, 92(3):1564-1568, 1992.

[37] Michael F Harrison. The misunderstood coagulopathy of liver disease: A review for the acute setting. Western Journal of Emergency Medicine, 19(5):863, 2018.

[38] Shu He, Aleksandra Antovic, and Margareta Blombäck. A simple and rapid laboratory method for determination of haemostasis potential in plasma: Ii. modifications for use in routine laboratories and research work. Thrombosis research, 103(5):355-361, 2001.

[39] Shu He, Katarina Bremme, and Margareta Blombäck. A laboratory method for determination of overall haemostatic potential in plasma. i. method design and preliminary results. Thrombosis research, 96(2):145-156, 1999.

[40] HC Hemker, P Giesen, R AlDieri, V Regnault, E De Smed, R Wagenvoord, T Lecompte, and S Beguin. The calibrated automated thrombogram (cat): a universal routine test for hyper-and hypocoagulability. Pathophysiology of haemostasis and thrombosis, 32(56):249-253, 2002.

[41] Erhard Hiller. Basic principles of hemostasis. In Modern Hematology, pages 327-345. Springer, 2007.

[42] McDonald K Horne III, Ann M Cullinane, Paula K Merryman, and Elizabeth K Hoddeson. The effect of red blood cells on thrombin generation. British journal of haematology, 133(4):403-408, 2006.

[43] Peter R. Hoskins. Physical properties of tissues relevant to arterial ultrasound imaging and blood velocity measurement. Ultrasound in Medicine and Biology, 33(10):15271539, OCT 2007.

[44] Chih-Chung Huang, Yi-Hsun Lin, Ting-Yu Liu, Po-Yang Lee, and Shyh-Hau Wang. Study of the blood coagulation by ultrasound. J Med Biol Eng, 31(2):79-86, 2011.

[45] Chih-Chung Huang, Shyh-Hau Wang, and Po-Hsiang Tsui. Detection of blood coagulation and clot formation using quantitative ultrasonic parameters. Ultrasound in medicine $\&$ biology, 31(11):1567-1573, 2005. 
[46] Angela Huth-Kühne, Francesco Baudo, Peter Collins, Jørgen Ingerslev, Craig M Kessler, Hervé Lévesque, Maria Eva Mingot Castellano, Midori Shima, and Jean St-Louis. International recommendations on the diagnosis and treatment of patients with acquired hemophilia a. haematologica, 94(4):566-575, 2009.

[47] NM Intagliata, CK Argo, JG Stine, T Lisman, SH Caldwell, F Violi, et al. Concepts and controversies in haemostasis and thrombosis associated with liver disease: Proceedings of the 7th international coagulation in liver disease conference. Thrombosis and haemostasis, 118(08):1491-1506, 2018.

[48] Craig M Jackson and M Peter Esnouf. Has the time arrived to replace the quick prothrombin time test for monitoring oral anticoagulant therapy?, 2005.

[49] William B Kannel. Overview of hemostatic factors involved in atherosclerotic cardiovascular disease. Lipids, 40(12):1215-1220, 2005.

[50] G.S. Kino. Acoustic Waves: Devices, Imaging, and Analog Signal Processing. PrenticeHall Contemporary Topics in Accounting Series. Prentice-Hall, 1987.

[51] Agnieszka Leszczyńska, Tomasz Misztal, Natalia Marcińczyk, Tomasz Kamiński, Karol Kramkowski, Ewa Chabielska, and Dariusz Pawlak. Effect of quinolinic acid-a uremic toxin from tryptophan metabolism-on hemostatic profile in rat and mouse thrombosis models. Advances in medical sciences, 64(2):370-380, 2019.

[52] James H Lewis. Comparative hemostasis in vertebrates. Springer Science \& Business Media, 2013.

[53] R. Libgot, F. Ossant, N. Desbuards, G. Y. Rochefort, D. Antier, Y. Gruel, and F. Patat. HF Ultrasound Device Designed to Describe the Whole Blood Coagulation Process: Application to Rats under Anticoagulant Therapy. In 2006 IEEE Ultrasonics Symposium, Vols 1-5, Proceedings, Ultrasonics Symposium, pages 622-625. IEEE, 2006.

[54] R Libgot, F Ossant, Y Gruel, P Lermusiaux, and F Patat. High frequency ultrasound characterization of the blood clotting process: Intra-and inter-individual variations. In IEEE Ultrasonics Symposium, 2005., volume 4, pages 2259-2262. IEEE, 2005.

[55] Rachel Libgot-Callé, Frédéric Ossant, Yves Gruel, Patrick Lermusiaux, and Frédéric Patat. High frequency ultrasound device to investigate the acoustic properties of whole blood during coagulation. Ultrasound in medicine $\& 3$ biology, 34(2):252-264, 2008. 
[56] H Roger Lijnen, Berthe Van Hoef, Veerle Beelen, and Désiré Collen. Characterization of the murine plasma fibrinolytic system. European journal of biochemistry, 224(3):863871, 1994.

[57] H Roger Lijnen, Berthe Van Hoef, and Désiré Collen. Characterization of the murine plasminogen/urokinase-type plasminogen-activator system. European journal of biochemistry, 241(3):840-848, 1996.

[58] Hui Yin Lim, Cindy O'Malley, Geoffrey Donnan, Harshal Nandurkar, and Prahlad Ho. A review of global coagulation assaysis there a role in thrombosis risk prediction? Thrombosis research, 2019.

[59] Rinske Loeffen, Kristien Winckers, Ian Ford, J Wouter Jukema, Michele Robertson, David J Stott, Henri M Spronk, Hugo ten Cate, Gordon D Lowe, and PROSPER Study Group. Associations between thrombin generation and the risk of cardiovascular disease in elderly patients: results from the prosper study. Journals of Gerontology Series A: Biomedical Sciences and Medical Sciences, 70(8):982-988, 2014.

[60] G Lutze, Jr G Lutze, K Kutschmann, and L Wiens. Plasma blood coagulation in mammals (domestic and zoo animals). experience with screening tests and determinations of individual factor activities. Hamostaseologie, 27(3):201-210, 2007.

[61] RG Macfarlane. An enzyme cascade in the blood clotting mechanism, and its function as a biochemical amplifier. Nature, 202(4931):498, 1964.

[62] Mary McGrae McDermott, Jack M Guralnik, Annamaria Corsi, Monique Albay, Claudio Macchi, Stefania Bandinelli, and Luigi Ferrucci. Patterns of inflammation associated with peripheral arterial disease: the inchianti study. American heart journal, 150(2):276281, 2005.

[63] Aurora M Morariu, Y John Gu, Rolf CG Gallandat Huet, Wout A Siemons, Gerhard Rakhorst, and Wim v Oeveren. Red blood cell aggregation during cardiopulmonary bypass: a pathogenic cofactor in endothelial cell activation? European journal of cardiothoracic surgery, 26(5):939-946, 2004.

[64] Marisa Ninivaggi, Rafael Apitz-Castro, Yesim Dargaud, Bas de Laat, H Coenraad Hemker, and Theo Lindhout. Whole-blood thrombin generation monitored with a calibrated automated thrombogram-based assay. Clinical chemistry, 58(8):1252-1259, 2012.

[65] Josune Orbe, Maite Zudaire, Rosario Serrano, Isabel Coma-Canella, Sara Martínez de Sizarrondo, Jose A Rodríguez, and Jose A Páramo. Increased thrombin generation 
after acute versus chronic coronary disease as assessed by the thrombin generation test. Thrombosis and haemostasis, 99(02):382-387, 2008.

[66] F Ossant, R Libgot, P Coupe, P Lerrnusiaux, and F Patat. Highfrequency ultrasound characterization of the coagulation process of whole blood. In Yuhas, MP, editor, 2004 IEEE Ultrasonics Symposium, Vols 1-3, ULTRASONICS SYMPOSIUM, pages 846-849. IEEE Ultrason Ferroelect \& Frequency Control Soc, 2004.

[67] K John Pasi. Hemostasis. In Hemostasis and thrombosis protocols, pages 3-21. Springer, 1999.

[68] V Peyrou, JC Lormeau, JP Herault, C Gaich, AM Pfliegger, and JM Herbert. Contribution of erythrocytes to thrombin generation in whole blood. Thrombosis and haemostasis, 81(03):400-406, 1999.

[69] C. Plag, R. Libgot, Y. Gruel, F. Patat, and F. Ossant. High frequency ultrasound characterization of blood clotting process : Results obtained with plasma and whole blood. In 2007 IEEE Ultrasonics Symposium Proceedings, Vols 1-6, Ultrasonics Symposium, pages 884+. IEEE, IEEE, 2007. IEEE Ultrasonics Symposium, New York, NY, OCT 28-31, 2007.

[70] Camille Plag, Yassine Mofid, Tony Matéo, Rachel Callé, and Frédéric Ossant. High frequency ultrasound imaging of whole blood gelation and retraction during in vitro coagulation. The Journal of the Acoustical Society of America, 131(5):4196-4202, 2012.

[71] Public Health Agency of Canada. Leading causes of deaths, Canada 2009/10. Pub.: 120179, Cat.: HP32-5/2010E-PDF.

[72] Public Health Agency of Canada. Leading causes of hospitilizations, Canada 2009/10. Pub.: 120179, Cat.: HP32-5/2010E-PDF.

[73] MH Qari. High throughput coagulation analyzers review. Combinatorial chemistry \& high throughput screening, 8(4):353-360, 2005.

[74] SM Razavian, D Quemada, M Th Guillemin, Y Beuzard, and M Boynard. Contribution of an ultrasonic interferometry method (echocell) to the determination of red blood cell sedimentation rate at low hematocrit. Journal de Physique III, 5(6):903-912, 1995.

[75] Caroline J Reddel, Jennifer L Curnow, Jasmin Voitl, Alexander Rosenov, Gabrielle J Pennings, Marie-Christine Morel-Kopp, and David B Brieger. Detection of hypofibrinolysis in stable coronary artery disease using the overall haemostatic potential assay. Thrombosis research, 131(5):457-462, 2013. 
[76] Min Rui, Wolfgang Bost, Eike C Weiss, Robert Lemor, and Michael C Kolios. Photoacoustic microscopy and spectroscopy of individual red blood cells. In Biomedical Optics, page BSuD93. Optical Society of America, 2010.

[77] J. Evan Sadler. Biochemistry and genetics of von Willebrand factor. Annual Review of Biochemistry, 67(1):395-424, 1998.

[78] D Schneditz, H Heimel, and H Stabinger. Sound speed, density and total protein concentration of blood. Clinical Chemistry and Laboratory Medicine, 27(10):803-806, 1989.

[79] W Secomski, A Nowicki, and P Tortoli. Estimation of hematocrit by means of attenuation measurement of ultrasonic wave in human blood. In Yuhas, DE and Schneider, SC, editor, 2001 IEEE Ultrasonics Symposium Proceedings, Vols 183 2, Ultrasonics Symposium, pages 1277-1280. Ultrason, Ferrelect, \& Frequency Control Soc, IEEE, 2001.

[80] KP Shung, RA Sigelmann, and G Schmer. Ultrasonic measurement of blood-coagulation time. IEEE Transactions on Biomedical Engineering, BM22(4):334-337, 1975.

[81] Mika Skeppholm, Anders Kallner, Karin Malmqvist, Margareta Blombäck, and Håkan Wallén. Is fibrin formation and thrombin generation increased during and after an acute coronary syndrome? Thrombosis research, 128(5):483-489, 2011.

[82] Machiel Smid, Arne WJH Dielis, Henri MH Spronk, Ann Rumley, Rene van Oerle, Mark Woodward, Hugo ten Cate, and Gordon Lowe. Thrombin generation in the glasgow myocardial infarction study. PloS one, 8(6):e66977, 2013.

[83] GG Stokes. On the effect of internal friction of fluids on the motion of pendulums. Trans. Camb. phi1. Soc., 9(8):106, 1850.

[84] Vacis Tatarunas, Algirdas Voleisis, Reimondas Sliteris, Rymantas Kazys, Liudas Mazeika, and Vaiva Lesauskaite. A novel ultrasonic method for evaluation of blood clotting parameters. Journal of Medical Ultrasonics, 45(4):545-553, 2018.

[85] AA Tokarev, AA Butylin, and FI Ataullakhanov. Platelet adhesion from shear blood flow is controlled by near-wall rebounding collisions with erythrocytes. Biophysical journal, 100(4):799-808, 2011.

[86] Federica Tosi, Roberta Micaglio, Marco Sandri, Annalisa Castagna, Diego Minguzzi, Filippo Stefanoni, Carmela Chiariello, Ilaria Franzese, Giovanni Battista Luciani, Giuseppe Faggian, et al. Increased plasma thrombin potential is associated with stable coronary 
artery disease: An angiographically-controlled study. Thrombosis research, 155:16-22, 2017.

[87] Armando Tripodi, Ida Martinelli, Veena Chantarangkul, Tullia Battaglioli, Marigrazia Clerici, and Pier Mannuccio Mannucci. The endogenous thrombin potential and the risk of venous thromboembolism. Thrombosis research, 121(3):353-359, 2007.

[88] Ioanna Tzoulaki, Gordon D Murray, Amanda J Lee, Ann Rumley, Gordon DO Lowe, and F Gerald R Fowkes. Inflammatory, haemostatic, and rheological markers for incident peripheral arterial disease: Edinburgh artery study. European heart journal, 28(3):354$362,2007$.

[89] Benjamin Valente-Acosta, Manuel Alfonso Baños-González, Marco Antonio PeñaDuque, Marco Antonio Martínez-Ríos, Leslie Quintanar-Trejo, Gad Aptilon-Duque, Mirthala Flores-García, David Cruz-Robles, Guillermo Cardoso-Saldaña, and Aurora de la Peña-Díaz. Association between stable coronary artery disease and in vivo thrombin generation. Cardiology research and practice, 2016, 2016.

[90] Shyh-Hau Wang, Tze-Wen Chung, Chun-Sheng Huang, Chin-Tang Chuang, and PoShiang Tsui. Detection of the process of blood coagulation and clot formation using quantitative ultrasonic parameters. In 2002 IEEE Ultrasonics Symposium, 2002. Proceedings., volume 2, pages 1653-1656. IEEE, 2002.

[91] R Wennesland, E Brown, J Hopper, JL Hodges, OE Guttentag, KG Scott, IN Tucker, and B Bradley. Red cell, plasma and blood volume in healthy men measured by radiochromium (cr51) cell tagging and hematocrit - influence of age somatotype and habits of physical activity on the variance after regrassion of volumes to height and weight combined. Journal of Clinical Investigation, 38(7):1065-1077, 1959.

[92] Matthew F Whelihan and Kenneth G Mann. The role of the red cell membrane in thrombin generation. Thrombosis Research, 131(5):377-382, 2013.

[93] Matthew F Whelihan, Vicentios Zachary, Thomas Orfeo, and Kenneth G Mann. Prothrombin activation in blood coagulation: the erythrocyte contribution to thrombin generation. Blood, 120(18):3837-3845, 2012.

[94] World Health Organization. Global Health Estimates 2016: Deaths by Cause, Age, Sex, by Country and by Region, 2000-2016. World Health Organization, 2018. 